2. To: (Receiving Organization)

SNF Project-2HAOO

5. Proj./Prog./Dept./Div.:

Waste Management Projects

8. Originator Remarks:

Data Quality objective document developed for disposal to ERDF of waste resulting from the $\mathrm{K}$ Basins Interim Remedial Action CERCLA ROD (EPA 541-R99-059)
11. Receiver Remarks:
11A. Design Baseline Document? $\square$ Yes $\bar{X}$ No

4. Related EDT No.:
7. Purchase Order No.:
9. Equip./Component No.:

10. System/BIdg./Facility:

12. Major Assm. Dwg. No.:

13. Permit/Permit Application No.:

14. Required Response Date:

May 3, 2000

\begin{tabular}{|c|c|c|c|c|c|c|c|c|}
\hline 15. & & DATA TR & NSMITTE & & (F) & (G) & $(\mathrm{H})$ & (l) \\
\hline $\begin{array}{l}\text { (A) } \\
\text { Item } \\
\text { No. }\end{array}$ & (B) Document/Drawing No. & $\begin{array}{l}\text { (C) Sheet } \\
\text { No: }\end{array}$ & $\begin{array}{l}\text { (D) Rev. } \\
\text { No. }\end{array}$ & (E) Title or Description of Data Transmitted & $\begin{array}{l}\text { Approval } \\
\text { Desig- } \\
\text { nator }\end{array}$ & $\begin{array}{c}\text { Reason } \\
\text { for Trans- } \\
\text { mittal }\end{array}$ & $\begin{array}{c}\text { Origi- } \\
\text { nator } \\
\text { Dispo- } \\
\text { stion }\end{array}$ & $\begin{array}{l}\text { Receiv- } \\
\text { er } \\
\text { Dispo- } \\
\text { sition }\end{array}$ \\
\hline 1 & $\mathrm{HNF}-6273$ & & 0 & Data Quality Objectives & ESA & 1 & 1 & \\
\hline & & & & Process for Designation of & & & & \\
\hline & & & & K Basin Debris & & & & \\
\hline & & & & & & & & \\
\hline & & & & & & & & \\
\hline & & & & & & & & \\
\hline & & & & & & & & \\
\hline
\end{tabular}

16.

KEY

\begin{tabular}{|c|l|}
\hline Approval Designator (F) & \\
\hline E, S, Q, D OR N/A & 1. Approval \\
(See WHC-CM-3-5, & 2. Release \\
Sec. 12.7) & 3. Information \\
\hline
\end{tabular}

17.

Reason for Transmittal (G)

4. Review

5. Post-Review

6. Dist. (Receipt Acknow. Required)

SIGNATURE/DISTRIBUTION

(See Approval Designator for required signatures)

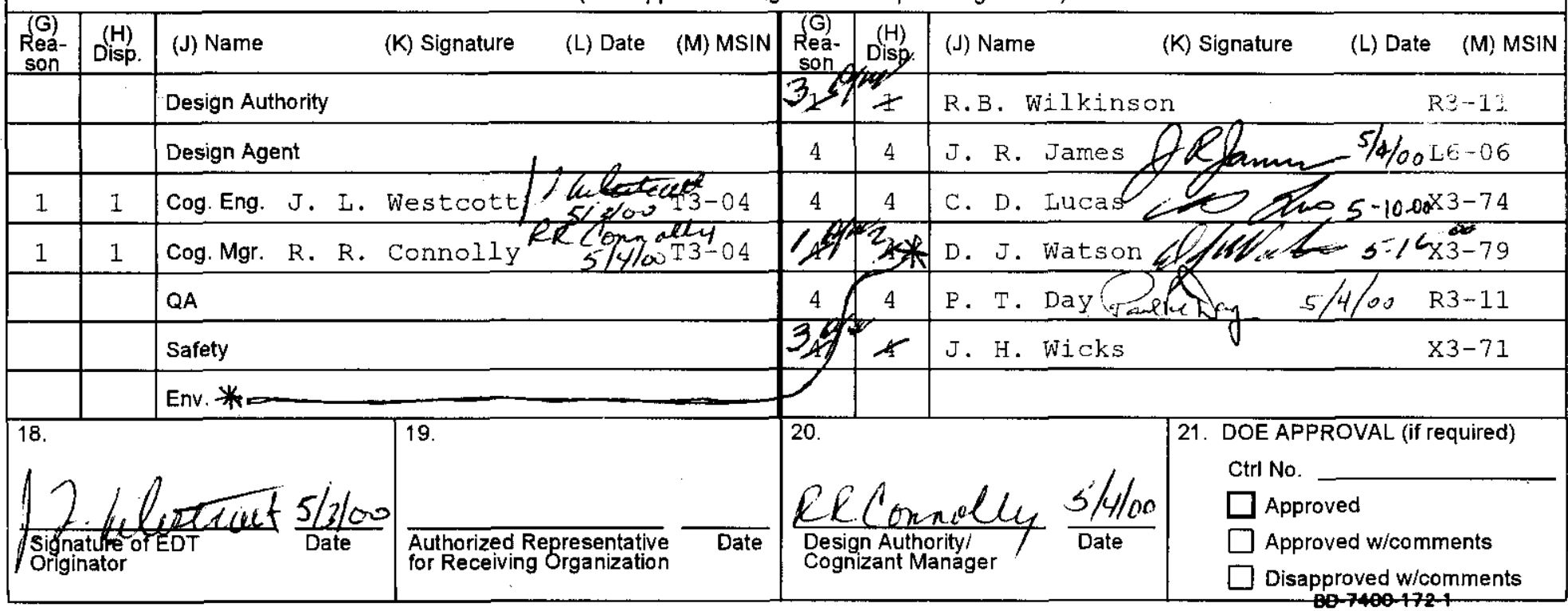

Disposition $(\mathrm{H}) \&(\mathrm{l})$

$\begin{array}{ll}\text { 1. Approved } & \text { 4. Reviewed no/comment } \\ \text { 2. Approved w/comment } & \text { 5. Reviewed w/comment }\end{array}$

$\begin{array}{ll}\text { 2. Approved w/comment } & \text { 5. Reviewed w/comment } \\ \text { 3. Disapproved w/comment } & 6 \text {. Receipt acknowledged }\end{array}$ 
HNF-6273

Revision 0

\section{Data Quality Objectives Process for Designation of K-Basins Debris}

Prepared for the U.S. Department of Energy

Assistant Secretary for Environmental Management

Project Hanford Management Contractor for the

U.S. Department of Energy under Contract DE-AC06-96RL13200

Fluor Hanford

P.O. Box 1000

Richland, Washington 
HNF-6273

Revision 0

EDT 621183

\title{
Data Quality Objectives Process for Designation of K-Basins Debris
}

\author{
J. L. Westcott
}

Fluor Hanford

Date Published

May 2000

Prepared for the U.S. Department of Energy

Assistant Secretary for Environmental Management

Project Hanford Management Contractor for the

U.S. Department of Energy under Contract DE-AC06-96RL13200

Fuor Hanford

P.O. Box 1000

Richland, Washington
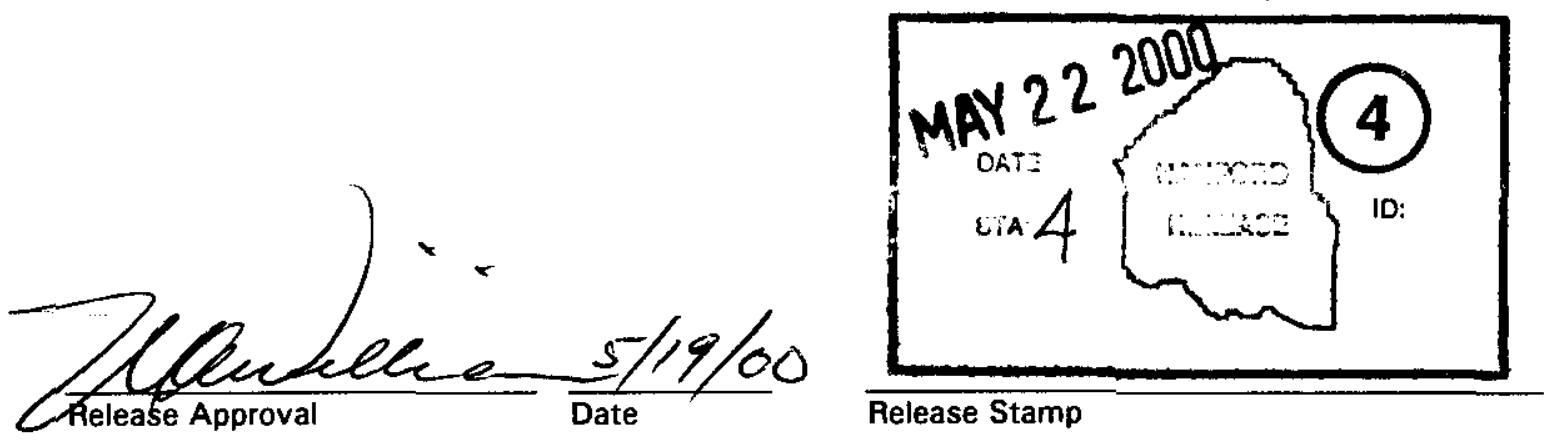
TRADEMARK DISCLAIMER

Reference herein to any specific commercial product, process, or service by trade name, trademark, manufacturer, or otherwise, does not necessarily constitute or imply its endorsement, recommendation, or favoring by the United

States Government or any agency thereof or its contractors or subcontractors.

This report has been reproduced from the best available copy.

Printed in the Unlted States of America

Total Pages: 119 
HNF-6273

Rev. 0

\section{EXECUTIVE SUMMARY}

The data quality objectives process was applied to support disposition of debris to be removed from above and below water at the $\mathrm{K}$ East and $\mathrm{K}$ West Basins. The purpose of the analyses discussed in this document is to designate debris removed from the $\mathrm{K}$ Basins to determine whether it is suitable for disposal at the Environmental Restoration Disposal Facility.

The structures that house the basins are classified as radioactive material areas. Therefore, all materials removed from the buildings are presumed to be radioactively contaminated. Because most of the materials that will be addressed under this plan will be removed from the basins, and because of the cost associated with screening materials for release, it is anticipated that all debris will be managed as low-level waste. Materials will be surveyed, however, to estimate radionuclide content for disposal and to determine that the debris is not contaminated with levels of transuranic radionuclides that would designate the debris as transuranic waste.

Debris that is contaminated with Resource Conservation and Recovery Act of 1976 I Washington State dangerous constituents above regulated levels will designate as mixed waste. Contamination may be present at levels that require treatment to comply with Land Disposal Restrictions. Debris $>60 \mathrm{~mm}$ that requires treatment for compliance with the Land Disposal Restrictions will be treated through macro-encapsulation as an approved alternative treatment technology for debris under 40 Code of Federal Regulations 268.45. Debris $\leq 60 \mathrm{~mm}$ will be treated as appropriate, based on Resource Conservation and Recovery Act of 1976 constituents. This approach is anticipated for only a small volume of debris and is more cost-effective than sampling this waste.

The sampling design for the debris incorporates two stages. In Stage 1, facility or historical radiological sample data will be used to establish the radionuclide/isotopic distribution of radiological constituents of concern. The radionuclide distributions will be established for each waste stream and subsequently used to estimate the content of constituents of potential concern, indexed to cesium-137. The cesium-137 content of 
Rev. 0

the waste will be estimated using a variety of instruments, including portable radiation detectors and nondestructive analysis (gamma spectroscopy, neutron counting) equipment. During Stage 2, K-Basin staff will use the correlation when evaluating data from radiological nondestructive analysis, dose rate, or gamma surveys to estimate isotopic inventories for waste shipments. 


\section{CONTENTS}

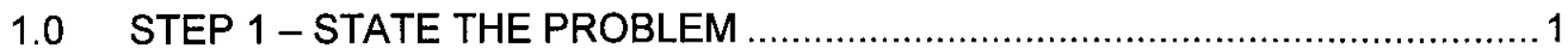

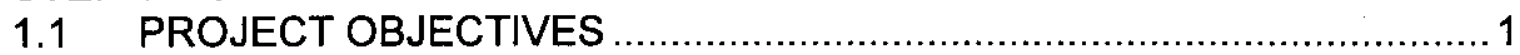

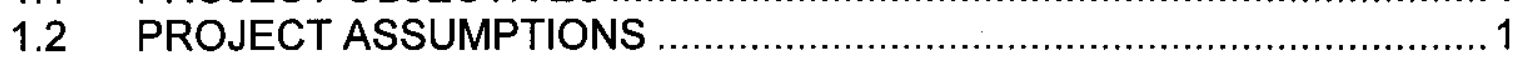

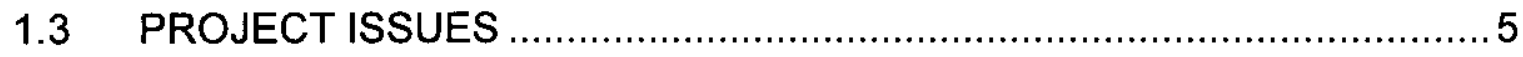

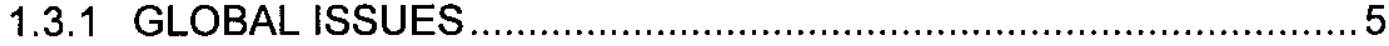

1.3.2 PROJECT-SPECIFIC TECHNICAL ISSUES

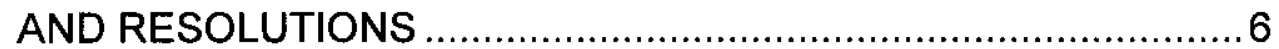

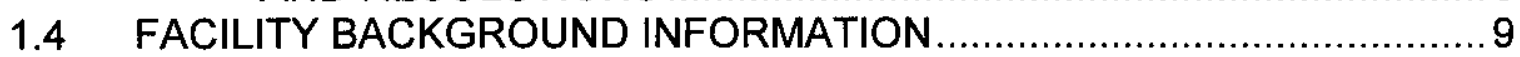

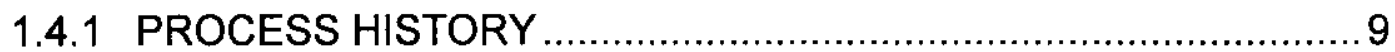

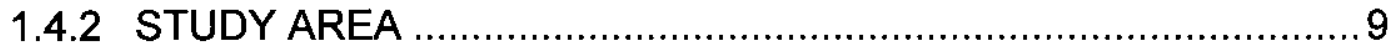

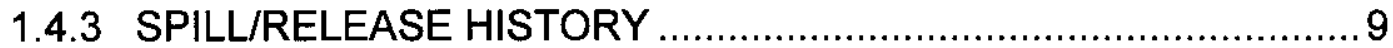

1.4.4 GENERAL HOUSEKEEPING PRACTICES ............................ 10

1.4.5 SUMMARY OF HISTORICAL DATA …................................. 10

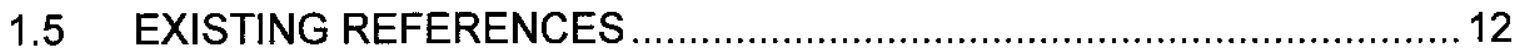

1.6 DQO TEAM MEMBERS AND KEY DECISION MAKERS .................... 14

1.7 CONTAMINANTS OF POTENTIAL CONCERN ....................................15

1.7.1 MASTER LIST OF CONTAMINANTS OF

POTENTIAL CONCERN ….............................................. 15

1.7.2 EXCLUDED CONTAMINANTS OF POTENTIAL CONCERN .......15

1.7.3 FINAL CONTAMINANTS OF CONCERN LIST .......................15

$1.8 \quad$ PRELIMINARY ACTION LEVELS ..............................................16

1.9 STATEMENT OF THE PROBLEM ............................................... 16

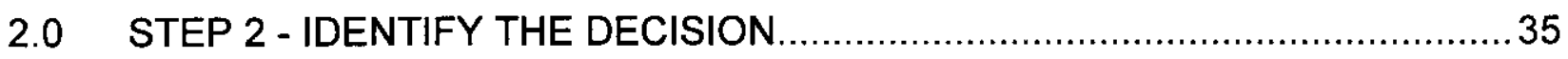

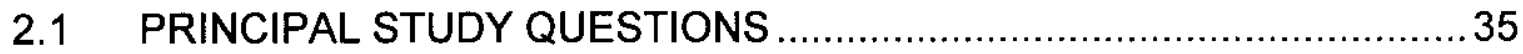

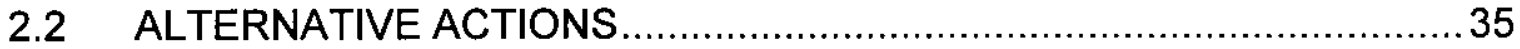

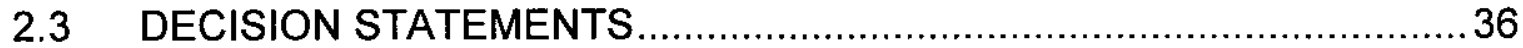

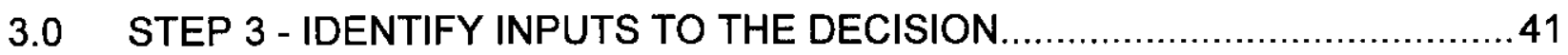

3.1 REQUIRED INFORMATION .......................................................

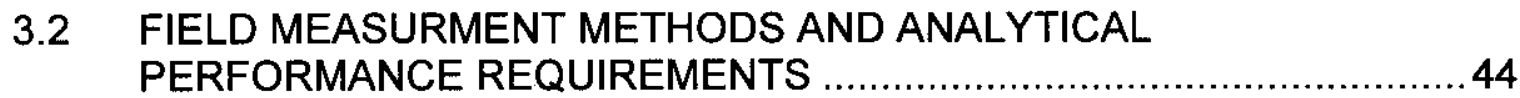

4.0 STEP 4 - DEFINE THE BOUNDARIES OF THE STUDY …..........................49

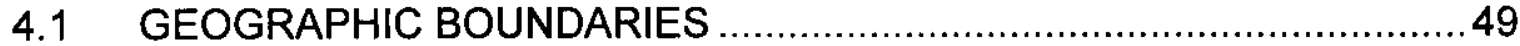

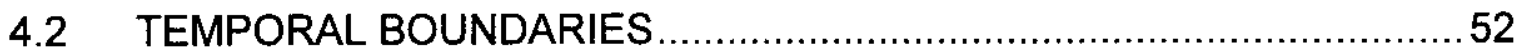

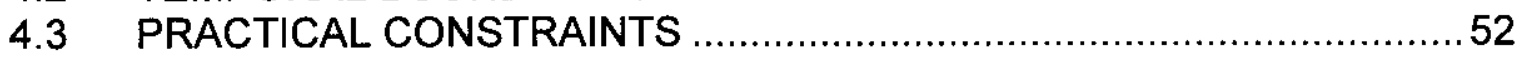

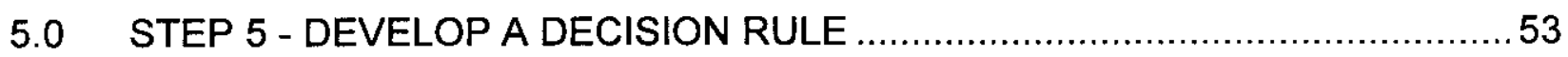

$5.1 \quad$ STATISTICAL PARAMETER OF INTEREST .....................................5

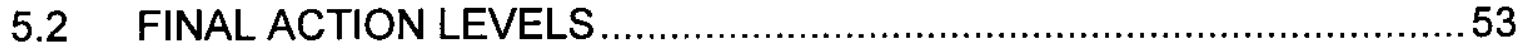

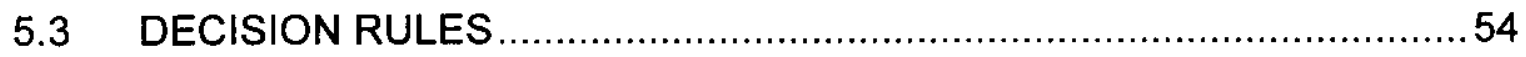


HNF-6273

Rev. 0

6.0 STEP 6 - SPECIFY TOLERABLE LIMITS ON DECISION ERRORS ................57

6.1 STATISTICAL VS. JUDGMENTAL DESIGN ................................57

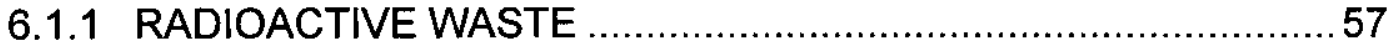

6.1.2 POTENTIALLY CHEMICALLY CONTAMINATED WASTE ..........58

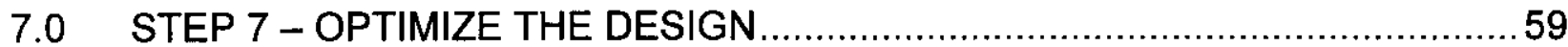

7.1 MATERIAL (COMPONENT) CATEGORIES ..................................5

7.2 SURVEYISAMPLING DESIGN ALTERNATIVES …........................59

7.3 SELECTION OF THE SURVEY/SAMPLING DESIGN ......................59

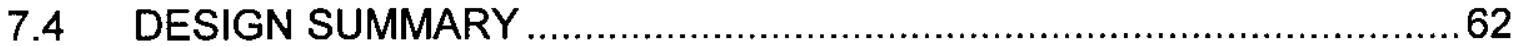

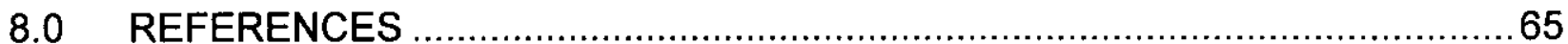

\section{APPENDICES}

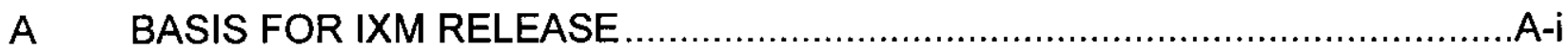

B RADIONUCLIDE/ISOTOPES COPC SELECTION .......................................

C ESTIMATION OF METALS AND PCBs SORBED ONTO ION-EXCHANGE

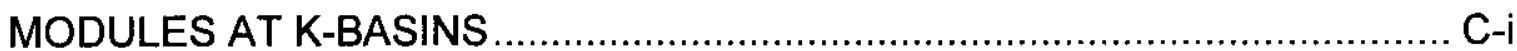

D DRAFT WASTE PROFILE CONTAINING DATA COLLECTED

AFTER SPRAY WASHING TO REMOVE PBV/TC METALS. ONLY APPENDIXES B \& F FROM PROFILE APPLY AND ARE INCLUDED.............. D-i

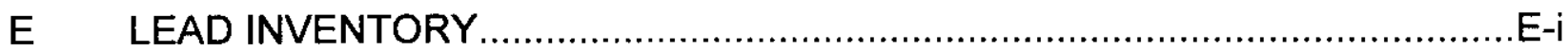

\section{FIGURES}

2-1. Generic Waste Disposition Decision Logic...............................................38

2-2. Chemical Waste Designation Decision Logic............................................ 39

\section{TABLES}

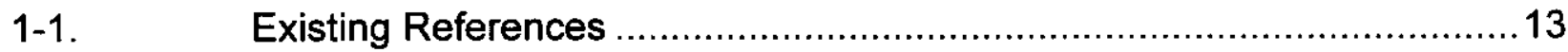

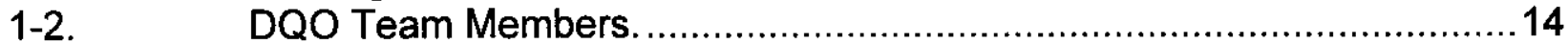

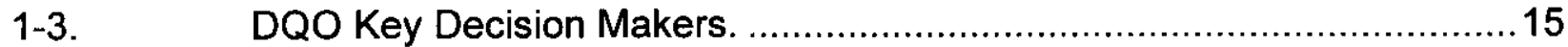

1-4. Master List of COPCs for Each Component or Waste Stream ...............17

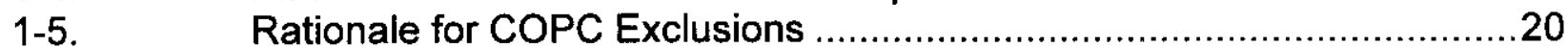

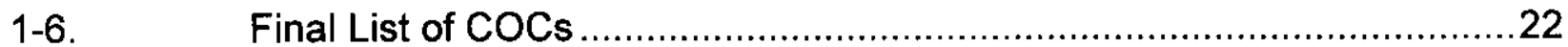

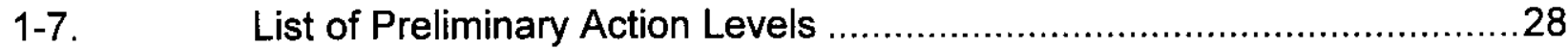

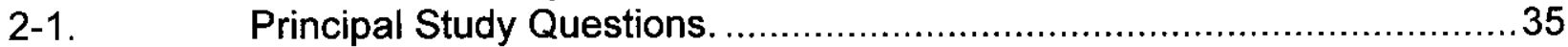


HNF-6273

Rev. 0

$2-2$.

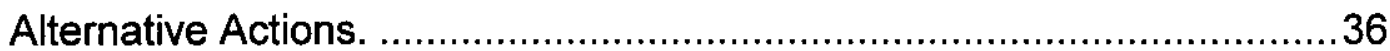

$2-3$.

Decision Statements.

3-1.

Required Information and Reference Sources.

3-1a.

Waste Designation Inputs

Information Required to Resolve the Decision Statements

Potentially Appropriate Measurement Methods.

Strata with Homogeneous Characteristics.

Final Action Level for the Decision. 
HNF-6273

Rev. 0 
HNF-6273

Rev. 0

\section{ACRONYMS}

ACM

$\mathrm{BHI}$

CERCLA

CFR

COCs

COPCs

CWC

DOE

DQO

DR

DS

EHW

EPA

EQM

ERDF

GEA

HEPA

HIC

ICP

IWTS

IX

IXM

$\mathrm{KE}$

KW

LDR

LLBG

LLW

MDL

NDA

PCB

PPE

PQL

PSQ

RCRA

ROD

SAP

SNF

TBD

TC

TCLP

TRU

TSCA

WAC

WS asbestos-containing material

Bechtel Hanford, Inc.

Comprehensive Environmental Response, Compensation and

Liability Act of 1980

Code of Federal Regulations

contaminants of concern

contaminants of potential concern

Central Waste Complex

U.S. Department of Energy

data quality objective

decision rule

decision statement

extremely hazardous waste

U.S. Environmental Protection Agency

Environmental Quality Management

Environmental Restoration Disposal Facility

gamma energy analysis

high-efficiency particulate air

high integrity container

inductively coupled plasma

integrated water treatment system

ion exchange

ion-exchange module

K East

K West

Land Disposal Restrictions

low-level burial ground

low-level waste

minimum detection limit

nondestructive assay

polychlorinated biphenyl

personal protective equipment

practical quantitation limit

principal study question

Resource Conservation and Recovery Act of 1976

Record of Decision

Sampling and Analysis Plan

spent nuclear fuel

to be decided

toxicity characteristic

Toxicity Characteristic Leachate Procedure

transuranic

Toxic Substances Control Act of 1976

waste acceptance criteria

waste stream 
HNF-6273

Rev. 0 
HNF-6273

Rev. 0

METRIC CONVERSION CHART

The following conversion chart is provided to aid the reader in conversion.

\begin{tabular}{|c|c|c|c|c|c|}
\hline \multicolumn{3}{|c|}{ Into Metric Units } & \multicolumn{3}{|c|}{ Out of Metric Units } \\
\hline If You Know & Multiply By & To Get & If You Know & Multiply By & To Get \\
\hline Length & & & Length & & \\
\hline inches & 25.4 & millimeters & millimeters & 0.039 & inches \\
\hline inches & 2.54 & centimeters & centimeters & 0.394 & inches \\
\hline feet & 0.305 & meters & meters & 3.281 & feet \\
\hline yards & 0.914 & meters & meters & 1.094 & yards \\
\hline miles & 1.609 & kilometers & kilometers & 0.621 & miles \\
\hline Area & & & Area & & \\
\hline sq. inches & 6.452 & sq. centimeters & sq. centimeters & 0.155 & sq. inches \\
\hline sq. feet & 0.093 & sq. meters & sq. meters & 10.76 & sq. feet \\
\hline sq. yards & .0836 & sq. meters & sq. meters & 1.196 & sq. yards \\
\hline sq. miles & 2.6 & sq. kilometers & sq. kilometers & 0.4 & sq. miles \\
\hline acres & 0.405 & hectares & hectares & 2.47 & acres \\
\hline Mass (weight) & & & Mass (weight) & & \\
\hline ounces & 28.35 & grams & grams & 0.035 & ounces \\
\hline pounds & 0.454 & kilograms & kilograms & 2.205 & pounds \\
\hline ton & 0.907 & metric ton & metric ton & 1.102 & ton \\
\hline Volume & & & Volume & & \\
\hline teaspoons & 5 & milliiters & milliliters & 0.033 & fluid ounces \\
\hline tablespoons & 15 & milliliters & liters & 2.1 & pints \\
\hline fluid ounces & 30 & milliliters & liters & 1.057 & quarts \\
\hline cups & 0.24 & liters & liters & 0.264 & gallons \\
\hline pints & 0.47 & liters & cubic meters & 35.315 & cubic feet \\
\hline quarts & 0.95 & liters & cubic meters & 1.308 & cubic yards \\
\hline gallons & 3.8 & liters & & & \\
\hline cubic feet & 0.028 & cubic meters & & & \\
\hline cubic yards & 0.765 & cubic meters & & & \\
\hline Temperature & & & Temperature & & \\
\hline Fahrenheit & $\begin{array}{l}\text { subtract } 32 \text {, } \\
\text { then multiply } \\
\text { by } 5 / 9\end{array}$ & Celsius & Celsius & $\begin{array}{l}\text { multiply by } \\
9 / 5, \text { then add } \\
32\end{array}$ & Fahrenheit \\
\hline Radioactivity & & & Radioactivity & & \\
\hline picocuries & 37 & millibecquerel & millibecquerel & 0.027 & picocuries \\
\hline
\end{tabular}


HNF-6273

Rev. 0 
HNF-6273

Rev. 0

\subsection{STEP 1 - STATE THE PROBLEM}

\subsection{PROJECT OBJECTIVES}

The U.S. Department of Energy has developed a schedule and approach for the removal of spent fuels, sludge, and debris from the $K$ East (KE) and $K$ West (KW) Basins, located in the 100 Area at the Hanford Site. The project that is the subject of this data quality objective (DQO) process is focussed on the removal of debris from the $\mathrm{K}$ Basins and onsite disposal of the debris at the Environmental Restoration Disposal Facility (ERDF). This material previously has been dispositioned at the Hanford LowLevel Burial Grounds (LLBGs) or Central Waste Complex (CWC).

The goal of this DQO process and the resulting Sampling and Analysis Plan (SAP) is to provide the strategy for characterizing and designating the K-Basin debris to determine if it meets the Environmental Restoration Disposal Facility Waste Acceptance Criteria (WAC), Revision 3 (BHI 1998). A critical part of the DQO process is to agree on regulatory and WAC interpretation(s) to support preparation of the DQO workbook and SAP.

\subsection{PROJECT ASSUMPTIONS}

The KE and KW Basins contain spent nuclear fuel (SNF) and contaminated sludge, water, and debris. Previous studies have shown that sludge is present in significant volumes in the KE Basin, which results in potentially higher surface contamination concerns for debris from this basin due to contact with the sludge. The scope of this $\mathrm{DQO}$ includes only the characterization of the debris from the $\mathrm{K}$ Basins and immediately adjacent areas, to allow the SNF Project to assign the appropriate waste designation. The scope includes characterization for disposal of the ion-exchange modules (IXMs) from the integrated water treatment system (IWTS). Waste designation will allow a determination of the appropriate method for treatment, packaging, and transportation of the waste for disposal at ERDF. If the debris cannot meet ERDF WAC (BHI 1998), it will be transferred to a 200 Area waste management facility, including one or more of the following: CWC, Mixed Waste Trench (W-025), LLBG, Waste Receiving and Processing facility, or T Plant. In this event, the waste must meet the requirements of the Hanford Site Solid Waste Acceptance Criteria (HNF 1998).

The Declaration of the Record of Decision for DOE Hanford 100 Area (EPA et al. 1999) for the $\mathrm{K}$ Basin defines debris qualitatively as all solid waste from the removal of materials from KE and KW Basins excluding SNF, sludge, and water. The Focused Feasibility Study for the K Basins Interim Remedial Action (DOE-RL 1998) provides a working definition of debris as any solid with a size greater than $0.64 \mathrm{~cm}(.25 \mathrm{in}$.). The purpose of this size specification for debris is to provide criteria to segregate fuel fragments from basin sludge. The project working definition of debris, as used in both the ROD and the FSS, is not to be confused with the RCRA definition of debris provided in 40 CFR $268.2(\mathrm{~g})$. For purposes of establishing disposal requirements, RCRA defines debris as a solid material exceeding a $60 \mathrm{~mm}(2.34 \mathrm{in}$.) particle size. Thus, 
debris from the $\mathrm{K}$ Basins is subdivided into two categories, small debris (60 $\mathrm{mm}$ or less) that is subject to standard RCRA waste disposal requirements, and large debris (greater than $60 \mathrm{~mm}$ ) that is eligible for disposal under the RCRA debris requirements. All project debris will be managed as required by the RCRA Land Disposal Restrictions. Project debris includes items located both above and below the water in the basins, wastes generated from operation of the water and sludge treatment systems, and wastes generated during basin deactivation. Equipment that is not an integral part of the basin structures will be decontaminated as appropriate, removed from the basin, drained, packaged, and disposed of as debris. Decontamination of debris from within the basins will take place primarily through a high-pressure wash system before the material is removed from the basin water. The pressure wash is expected to remove the majority of sludge from the surface of the debris, thus eliminating the majority of surface contamination from radionuclides, polychlorinated biphenyls (PCBs), and regulated metals.

Equipment could include components of the SNF retrieval system and washing station, the IWTS, and the sludge retrieval system. The IWTS equipment and the structure in which it is installed will be removed, decontaminated as appropriate, packaged, and disposed as debris. Debris also includes the aluminum and stainless steel fuel canisters in the basins, fuel racks, and miscellaneous piping, tools, hose, scrap, and other materials. There are approximately 1,800 empty and 7,400 full canisters in the two basins with an estimated waste volume of $27,600 \mathrm{ft}^{3}$. Full canisters will be managed for disposal according to this DQO and resulting SAP only after the fuel has been removed. Fuel racks will make up approximately $1,546 \mathrm{ft}^{3}$ of waste, and miscellaneous debris from the two basins will result in approximately $1,289 \mathrm{ft}^{3}$ of waste (Remedial Design Report and Remedial Action Work Plan for the K Basins Interim Remedial Action (DOE-RL 1999a), Section 1.3.3 "Contaminated Debris").

Debris management will depend on the waste designation. Because the K-Basin structures are designated as a radioactive material area, all materials are anticipated to be low-level waste (LLW), unless they can be released through survey and analysis or contamination is detected that causes the material to be designated transuranic (TRU) waste. Debris might designate as LLW, mixed waste, TRU waste, or TRU mixed waste, depending on contaminant concentrations associated with the specific items.

Limited analysis of samples from the basins indicates the presence of PCBs in sludge from some locations. All debris will be pressure-washed and drained of free-flowing liquid as it is removed from the basins; after washing, the debris will not subsequently be regulated under the Toxic Substances Control Act of 1976 (TSCA $)^{1}$. Although the paint on some debris items may contain PCBs, the concentrations are assumed to be below levels of concern for disposal at ERDF (concentrations are based on the total

1 The K-Basin ROD (EPA et al. 1999) states that debris is regulated as PCB remediation waste where it has contacted sludge. After it is drained of free-flowing liquids and rinsed with water to remove the sludge, the debris will no longer be managed as TSCA-regulated waste. The risk-based disposal approval under 40 CFR 761.61(c) is based on the expectation that minimal quantities and concentrations of PCBs will be left on the debris and that management of this waste in accordance with applicable radioactive waste and dangerous waste requirements will be protective of residual PCBs present in the waste. 
HNF-6273

Rev. 0

mass for the item, not merely the paint itself). Some items, such as fluorescent light ballasts, are assumed to have regulated PCBs and are managed appropriately. For all these reasons, this DQO and resulting SAP do not include a sampling strategy for PCBs.

Before disposal at the ERDF, LLW from the K Basins might be crushed, sized, sorted, etc., to minimize volumes for disposal. Lead bricks and lead shielding will undergo macro-encapsulation before disposal at ERDF. Other debris that does not meet Land Disposal Restriction (LDR) criteria after decontamination also will be encapsulated for disposal at ERDF. Based on the debris inventory ( $K$ Basins Debris Inventory [Knox 1997]), a relatively small volume of painted debris is anticipated as part of the waste stream (WS). The SNF Project will establish a toxicity characteristic (TC) contaminant of concern (COC) to mass ratio for painted objects as part of the SAP. Painted debris will be assigned a TC designation for metals, based on the total mass of the object(s). The project believes that designation of this waste based on an agreed-upon ratio is a more efficient approach than sampling the painted debris for characterization. The same approach may be used for other small-volume suspect WSs, such as light bulbs. The overall quantity of hazardous constituents from these wastes is considered to be minimal. This approach is considered appropriate due to the low volume of waste, coupled with the expense of sampling and analysis, as compared to encapsulation. Macro-encapsulation is a designated alternative treatment standard for debris with no contaminant restrictions under 40 Code of Federal Regulations (CFR) 268.45.

Transuranic waste is not eligible for disposal at ERDF and will be directed to an alternate waste management pathway. All debris will be field surveyed for radionuclide contamination after pressure washing and removal from the basins. A small fraction of the debris might designate as either contact-handled or remote-handled TRU waste, or TRU mixed waste (waste that designates as both dangerous waste and TRU waste). This might happen if sludge or fuel particles are trapped inside debris. Debris with entrained sludge or fuel particles will be placed into a basket at the K Basins, agitated to dislodge the sludge and particles, and washed with water. Any debris that is still TRU-designated after this washing will be stored temporarily at the CWC until it can be packaged and certified at the Waste Receiving and Packaging facility for eventual disposal at Waste Isolation Project Plant. Debris that is not designated as TRU waste will be treated and packaged as appropriate and transferred to the 200 Area for disposal at ERDF. Actual treatment (e.g., macro-encapsulation) may take place at ERDF.

Six individual categories of waste have been identified by K-Basin project personnel for inclusion in ERDF waste profiles. The purpose of this document is to generate sufficient data to allow for the establishment of waste profiles. Although project personnel may wish to retain these categories for inventory control purposes, ERDF personnel will likely consolidate the waste categories into fewer profiles that correspond to the facility's waste management needs. The WSs identified by project personnel are described as follows: 
Mixed Waste - consists of radiologically-contaminated materials that are also regulated as hazardous waste under the Resource Conservation and Recovery Act of 1976 (RCRA) or dangerous wastes under the Washington State Dangerous Waste Regulations.

Above Water Waste - consists of radioactive, nonhazardous consumables such as disposable personal protective equipment (PPE) and other miscellaneous trash (e.g., paper, plastic, cloth, rubber, insulation, and light metal), as well as demolition debris such as structural steel and other fabricated components.

Underwater Debris - consists of radiologically-contaminated materials that are removed from beneath the water of the KE and KW Basins. These materials consist primarily of metals, plastic, and rubber components associated with tools, equipment, and structures used for handling, moving, and managing the submerged SNF. In the course of fuel handling activities, some of these items may have come into direct contact with the fuel elements (in the KW Basin) and with the exposed fuel and contaminated sludge in the KE Basin.

lon-exchange Modules - consists of the intact spent IXM assemblies from the K-Basin water treatment system. Ion-exchange modules are used to maintain the quality of the demineralized water in the basins. The IXMs remove dissolved radionuclides from the storage basin water. Each IXM consists of a block of concrete, with six steel columns cast into the concrete to contain the ion-exchange (IX) media. The Project and U.S. Environmental Protection Agency (EPA) staff are reevaluating the suitability of the IXMs, when properly drained, as being compliant with the 40 CFR 265.45 standard for macro-encapsulation and therefore being suitable for disposal at ERDF without additional treatment. This evaluation must be completed prior to disposal of the IXMs to ERDF; if compliance with the macro encapsulation requirement cannot be demonstrated, then alternative disposition of the IXMs will be pursued. An alternative disposition colud be revising the CERCLA ROD to provide a treatment variance for the LDR requirement. (See Appendix A)

Canisters - The SNF presently stored in the K Basins is contained in cylindrical metal canisters. The SNF is not subject to the discussions in this DQO. The fuel will be removed from the canisters as part of the fuel retrieval process and the empty canisters will become waste debris. This WS consists of the empty aluminum and stainless steel canisters.

Asbestos Debris - consists of construction materials with varying content of asbestos and asbestos-containing materials (ACMs). The age of the KE and KW Basin facilities indicates that asbestos is likely to be present in numerous materials. Asbestos debris may also be radiologically-contaminated, regulated as hazardous waste, or be mixed waste. 
HNF-6273

Rev. 0

\section{$1.3 \quad$ PROJECT ISSUES}

\subsubsection{Global Issues}

The following Global Issues, identified in interviews with project staff and decisionmakers, were discussed in a meeting with decision-makers.

1. Is the project decontaminating surfaces with anything other than "Citristrip" paint stripper and rags?

2. What are the physical boundaries of the debris removal? What is the volume of debris to be removed? How much PPE is forecast for disposal?

3. Polychlorinated biphenyls can be held on the IX column. The concentration of PCBs in the IX column and entire module has been calculated. Does the project want to calculate the concentration based on the column or the entire module? Does a vented but filtered, IXM comply with the requirement for macroencapsulation?

4. Pressure washing has been designated as the primary method of removing sludge contamination from below-water-level debris. How will the project verify that porous surfaces meet the ERDF WAC (BHI 1998)?

5. Nonradioactive metals will also be trapped on the IX column. The concentration of RCRA metals on the IX column has been calculated based on the water data and other information provided by the project. If these levels exceed the TC levels calculated based on total metals, how will LDR concentrations be addressed?

6. Previously, some mixed waste was designated as dangerous waste with a state code under the dangerous waste criteria per Washington Administrative Code 173-303-100. Will ERDF apply this code and is there any prohibition to accepting this code? Do all parties agree that dried paint should not include volatile organic constituents of wet paint when calculating this code?

7. For painted debris, do all parties agree to calculate the lead and other RCRA metals content based on the entire volume of debris disposed? Explain the weight percent that may cause the RCRA TC limits to be exceeded.

8. How will ballasts from fluorescent lights be disposed? (These may contain PCBs.)

9. Are the IXMs or any other waste TRU?

10. Do all parties agree that with respect to PCBs on painted debris, the waste is not TSCA based on the Record of Decision (ROD) (EPA et al. 1999)? The ROD appears to apply only to debris from below water level. How will debris above the water level be dealt with? 
11. Environmental Restoration Disposal Facility packaging specified in the ERDF WAC Supplement (Supplemental Waste Acceptance Criteria for Bulk Shipments to the Environmental Restoration Disposal Facility [BHI 1997]) has been accepted to meet the U.S. Department of Transportation requirements. Environmental Quality Management (EQM) has been told by ERDF that this is not necessarily the case. There is also a Safety Analysis Report for Packaging for the IX module. Will any packaging be used that does not fall into either of these categories?

12. Is the water filtered before the IX column? If so, how will filters be disposed?

13. Does the Comprehensive Environmental Response, Compensation and Liability Act of 1980 (CERCLA) ROD cover maintenance debris?

\subsubsection{Project-specific Technical Issues and Resolutions}

Global Issues were resolved in the following manner:

1. If a product other than Citristrip is used in the future, the constituents of those materials will be characterized and included in a waste profile at that time.

2. The EPA's perspective is that anything resulting from the CERCLA remedial action that is not sludge, water, or SNF is debris per the K-Basin ROD (EPA et al. 1999 ) and can be addressed by this plan. Some demolition debris, generated to allow equipment access to the basins, will be included. If necessary, the DQO and SAP will be amended to address additional concerns, such as sand from filters.

Profiles of waste going to ERDF will generally be bounded by waste type (hazardous and nonhazardous). Similar WSs will be allowed within each ERDF profile; however, hazardous and nonhazardous materials must be disposed under separate profiles. For example, painted concrete could be either hazardous or nonhazardous depending on lead concentration and mass of material disposed. Although the project may need to categorize by waste source for process control needs, this information is not required by ERDF.

Forecasts of waste volume have been made, but are by waste type, not the sources as listed above. There are approximately $30,000-40,000$ pounds of lead shielding bricks-four to five boxes worth. The ERDF macro-encapsulates the bricks/shielding at the disposal site before disposal.

3. All documents need to clearly define the IX column versus the IXM. The IXM includes the columns and the concrete and any valving, while the IX columns are simply the metal columns and the resins. 
The EPA's perspective on the IXM is that the unit, which includes the columns and concrete shell, is a high-integrity container (HIC). From the agency perspective, this waste is encapsulated and meets the $40 \mathrm{CFR}$ treatment standards for metals and PCBs. From the Agency's perspective, as long as the contents are not TRU, the unit can be sent to ERDF. The EPA interpretation letter supporting this position is included as Appendix $A$. The ERDF representatives will review this interpretation before committing to acceptance of the IXMs in this configuration. The project will proceed on the assumption that this approach will be used.

The EPA noted that there is no need to further characterize the resins, because the unit as defined in the ROD is considered debris. This raised a concern on the part of ERDF as to whether the concrete component of the IXM is part of the "debris" that is being disposed of, or the "encapsulation." Resins, on their own, would not meet the RCRA definition for debris. The EPA noted that this is part of the reason why debris was specifically defined differently in the ROD.

In response to a question of whether there is a potential for heat generation, it was noted that the total IXM package is high-efficiency particulate air (HEPA) vented. It was noted that the venting is primarily for release of hydrogen gas, not heat. The venting of the package raised concerns on the part of ERDF as to whether the debris is truly macro-encapsulated as defined in 40 CFR 268.45. Because the vent is for gas release, not heat release, it is possible that the vent could be sealed. This concern was addressed in the EPA letter, provided in Appendix $A$, which indicates that this configuration is acceptable for compliance with 40 CFR 268.45. [BHI subsequently evaluated the gas-generation issue and determined that it does not present a concern for disposal (see Appendix A).]

Ion-exchange columns that are stored within the K-Basin site are generally TRU waste and would not be considered for disposal to ERDF regardless. They are not being considered under this DQO.

4. According to the ROD (EPA et al. 1999), once an item is rinsed, PCBs are not a disposal issue. Bechtel Hanford, Inc. indicated that they would need to take a look at the specific treatment standards for the various forms of debris before agreeing that rinsing will address all concerns. The EPA reiterated that if there is a question as to whether an item exceeds regulated concentrations, the item can be designated as hazardous, placed in a container, and grouted (macroencapsulated). Based on the anticipated small volume of questionable debris and the cost and delay associated with characterization, the SNF Project believes this is an appropriate strategy. Debris will be designated based on process knowledge. Bechtel Hanford, Inc. raised a concern as to whether specific items will qualify as debris, as defined in 40 CFR 268 , and whether washing is sufficient treatment. Some items may be smaller than the regulatorydefined size for debris. 
5. Metals concentrations were derived based on assumptions that included maximum values at the level of detection in feed water. Metals may not be an issue if, based on recalculation of the values using newly provided data, concentrations drop below LDR limits. [Upon inspection of the new data, it was discovered that the values were provided for effluent water only, not intake water. Therefore, recalculation of concentrations was not appropriate or useful.] It was noted, however, that concentrations in feed water might increase over time. Macro-encapsulation of the IXM has been approved as treatment and may address concerns for disposal of this debris under LDR.

6. Although most parties agree that volatiles are not a concern for dried paint, $\mathrm{BHI}$ is looking for objective evidence that this is in fact the case. This evidence could be from analysis or from examination of other dried paints with similar volatile content. The EPA recommended consideration of the total weight of the painted item for purposes of waste designation. Stripped paint will be collected on rags and treated as part of the debris WS.

7. Metals content is based upon the mass of the debris disposed of, not just the paint layer.

8. The site has a fluorescent light recycling program. Most of the bulbs and ballasts will go to this program. Broken bulbs in contaminated areas will go into a box with other debris for grouting (macro-encapsulation) based on TC designation for lead and possibly mercury (for older bulbs).

9. Any TRU waste will not go to ERDF.

10. Polychlorinated biphenyl solids meeting the ERDF WAC (BHI 1998) can go to ERDF, so the described waste form is not a concern. [NOTE: the ERDF does not accept liquid PCBs.]

11. The Safety Analysis Report for Packaging applies only to IXMs. Environmental Restoration Disposal Facility roll-off boxes are approved as a U.S. Department of Transportation IP-1 package and are considered an IP-2 package within the Hanford Site barricades. Other containers can be approved for use inside the barricades. Characterization performed to meet disposal needs is generally considered adequate to meet transportation needs.

12. The basin water is filtered before IX column.

13. The CERCLA ROD (EPA et al. 1999) covers maintenance debris. 
HNF-6273

Rev. 0

\subsection{FACILITY BACKGROUND INFORMATION}

\subsubsection{Process History}

The KE and KW Reactors and their associated fuel storage basins were constructed in the early 1950s. The basins are located in the Hanford $100 \mathrm{~K}$ Area within $420 \mathrm{~m}$ $(1,380 \mathrm{ft})$ of the Columbia River. The fuel basins are large, open-topped concrete pools containing approximately 4.9 million liters ( 1.3 million gallons) of demineralized water. The basins were originally used to store SNF from the KE and KW Reactors until the early 1970 s, when these reactors were removed from service and the fuel removed from the basins. The KE and KW fuel storage basins were subsequently used to store SNF from the Hanford N Reactor. The KE and KW fuel basins currently hold approximately 1,200 metric tons and 900 metric tons of $N$ Reactor SNF, respectively. The spent fuel elements are contained in canisters placed in storage racks under $5 \mathrm{~m}$ (16 ft) of water for cooling and radiation shielding.

\subsubsection{Study Area}

The study area consists of the areas below water and above water areas adjacent to the KE and KW Basins which will be affected by debris removal activities. Debris includes all solid waste (including IXMs and canisters) generated during cleanup and deactivation activities. The total (compacted) volume of in-pool debris at the end of calendar year 1998 was estimated to be about $150 \mathrm{~m}^{3}\left(5,305 \mathrm{ft}^{3}\right)$ in KE Basin, and $125 \mathrm{~m}^{3}\left(4,400 \mathrm{ft}^{3}\right)$ in KW Basin (105 K-Basins 1998 Debris Report [DOE-RL 1999b]). Additional debris volumes will be generated as a result of construction, operations, and deactivation of the facilities in support of the CERCLA interim remedial action.

\subsubsection{Spill/Release History}

The basins contain SNF and contaminated sludge, water, and debris. A significant portion of the SNF in the KE Basin was damaged during discharge from the reactor, resulting in breaching of the fuel cladding and release of soluble radionuclides into the basin waters and sludge. Most of the shorter-lived radionuclides have deteriorated to low levels and no hazardous chemicals have been identified in the basin waters. The SNF in both basins is deteriorating under the current storage conditions. In addition, there have been at least two documented leaks of contaminated water from the $\mathrm{KE}$ Basin into the underlying soil and groundwater.

The present condition of the basins and the SNF lead to the implementation of a CERCLA interim remedial action on the K-Basins cleanout project and development of a CERCLA proposed plan and ROD for the cleanout. 
HNF-6273

Rev. 0

\subsubsection{General Housekeeping Practices}

Materials collected during general housekeeping and work area clean up, such as floor sweepings (soil, sawdust), vegetation debris, glass, plastic, and PPE and related material from personnel egress and decontamination areas, are included as K-Basin Debris for disposal at the ERDF. Paint and Citristrip waste, generated from the limited practice of paint stripping of debris to allow for welding activities, will be collected on rags. Based on the low concentration of paint waste on individual rags, rags will be collected and managed for disposal as debris at ERDF.

\subsubsection{Summary of Historical Data}

The Listed Waste History at Hanford Facility TSD Units (WHC 1996) will be reviewed as specific WSs are generated to verify that there are no listed waste concerns before designation. Sampling and analysis activities that have been performed to characterize waste materials from the $\mathrm{K}$ Basins are summarized briefly below:

Mixed Waste Debris - No waste-specific radiochemical laboratory analyses have been performed to date on this WS. For past shipments, an estimate of the cesium-137 content of the waste was performed using established dose-to-curie relationships (WHC 1996a, WHC 1996b). Once a measurement of dose or cesium-137 activity in the waste is obtained, radionuclide composition of the waste can be estimated by applying the radionuclide ratios measured and reported previously (DeVanney 1990). For radionuclides that were not analyzed in the referenced report, the radionuclide ratios in the Hanford Site Solid Waste Acceptance Criteria, Revision 4 (WHC 1993, Appendix K), provided estimates that were used. Radionuclides considered reportable in previous waste shipments included strontium-90, cesium-137, plutonium-239/240, americium-241, and plutonium-241. This entire WS was designated as low level radioactive mixed waste. Inductively coupled plasma total metals analysis (SW-846 Method 6010A [Test Methods for Evaluating Solid, Waste Physical/Chemical Methods, EPA 1997]) have been performed on nine paint chip samples as well as multiple chip samples from the overhead crane. Toxic metals (silver, arsenic, barium, cadmium, chromium, lead, and selenium) were confirmed to be present in paint chips at total concentrations greater than screening limits for the TC criteria. Toxicity Characteristic Leachate Procedure (TCLP) metals analyses were not conducted.

Above Water Waste - Radiochemical analyses for gross alpha, gross beta, cobalt-60, cesium-137, americium-241were performed on twenty 105-KE smears. A nondestructive analysis of 20 compacted drums was performed at Allied Technology Group, and nondestructive assay (NDA) of four boxes of waste was conducted on the $100 \mathrm{~K}$ Pad. Based on these analyses, radionuclides in previous waste profiles included strontium-90, cesium-137, europium-152, plutonium-238, plutonium-239, plutonium-240, americium-241, plutonium-241, and curium-244. All of this WS was designated as lowlevel radioactive waste with the exception of one barrel, which was estimated to potentially contain TRU waste. Nonradiological sampling was limited to the same paint chip samples used for characterizing the mixed waste debris. 
Underwater Debris - Radiochemical analyses were performed on coupons from pipes that were rinsed and removed from the basin. Analyses included total alpha, gamma energy analysis (GEA), strontium-89/90, americum-241, and total uranium.

Radionuclides that were found above detection limits included cesium-137, cobalt-60, europium-154/155, strontium-90, uranium, plutonium-238, 239/240, and americium-241. In addition, 11 boxes of rinsed debris that were on the $100 \mathrm{~K}$ Rad Pad were evaluated by NDA for maximum cesium-137 content. The radioactive constituents of the waste were estimated from these measurements. All of the waste was determined to be low-level radioactive waste.

Polychlorinated biphenyl analysis was conducted on waters from the KE and KW Basins; PCBs were not detected using a minimum detection limit (MDL) of $0.5 \mathrm{ug} / \mathrm{ml}$. Inductively coupled plasma (ICP) analysis for total metals was performed on water samples from both basins and on sludge from the KE Basin only. Although zinc, silicon, copper, and boron were detected in water samples, no TC metals were found using MDLs less than the TC levels. Metals have been found in KE-Basin sludge at concentrations that exceed the total concentration screening level. No TCLP analyses were performed on the sludge.

Canisters - In 1996, several empty fuel canisters were pressure washed and removed from the basin for characterization (Characterization of Empty Fuel Canisters in 105 KE Basin [WHC 1996f]). Smears were obtained from the canisters and submitted for GEA. The pressure-washed canisters were analyzed by NDA (gamma and neutron analysis) and an estimate was performed of the radionuclide content of the canisters. The NDA results reported in WHC $(1996 \mathrm{~g})$ indicated that the rinsed canisters were contaminated with estimated concentrations of cesium-137, cobalt-60, amercium-241, europium-154, 155, antimony-125 and potassium-40. The conclusion of the report was that the pressure washed canisters were not TRU waste. The report estimated the TRU content of the pressure washed canisters based on the NDA gamma analytical results and results of smears.

The NDA did not report any americium-241 or plutonium-239/240. Subsequent laboratory analysis of smears taken from nine of the 11 canisters that were subjected to NDA demonstrated a significant americium-241 content (up to $41 \%$ of the measured cesium-137 activity). The smears were analyzed in the laboratory; however, they were only subjected to gamma analysis and, thus, did not detect any plutonium isotopes. The lack of apparent plutonium-239/240 was explained in the report by a hypothesis that the americium-241 reacted with the underlying canister metal while the plutonium isotopes were associated more with the sludge that was presumed to be washed off. No data were presented to substantiate that hypothesis.

Evaluation of the gross alpha data from the smears demonstrated that, in general, the reported americium-241 activity of the smears was around 20 to $40 \%$ of the gross alpha activity. This leaves 60 to $80 \%$ of the alpha activity unaccounted for. Evaluation of the radiochemistry data from pipe samples, sludge, basin water, and fuel rod radionuclide content do not support a high degree of enrichment of americium- 241 relative to 
cesium-137 without a concomitant presence of plutonium isotopes. An estimate of the detection limit for plutonium-239 in 1-gallon cans indicated that the method could, at best, detect approximately $0.025 \mathrm{~g}$ of plutonium-239. The estimated levels of plutonium-239 that would be anticipated in the canisters based on measured ratios of radionuclides in other metallic samples, sludge, and water are generally below that level. The amount of plutonium-239 that would be estimated in the canisters (based on ratios of plutonium to americium found in other below water waste samples) would range from 0.0015 to $0.049 \mathrm{~g}$ plutonium.

The estimate of TRU on the canisters and other below water debris did not include an estimated plutonium isotope component and is identified as a data gap.

Asbestos - No radiochemical or chemical analyses have been performed. It is anticipated that the radionuclide content of the asbestos waste will be estimated by the same approach used for the above water waste.

lon-exchange Modules - The radionuclide content of the IXMs was estimated from analysis of the basin water and an assumption that $100 \%$ of the radionuclides measured in the water are removed by the IXM. Radionuclides are routinely measured in the basin water and those routinely detected included americium-241, cesium-137, strontium-90, plutonium-238, plutonium-239/240, and uranium. No radiochemistry measurements have been performed on the actual IX material due to as low as reasonably achievable (ALARA) considerations. Ion-exchange modules are routinely removed from service prior to reaching a loading that could result in the IXM being declared a TRU waste (SNF Project 2000). Polychlorinated biphenyls were not detected in K-Basin water above the $0.5 \mathrm{ug} / \mathrm{ml}$ level. Toxic metals were undetected in K-Basin water (MDLs were less than TC levels); only zinc, silicon, copper, and boron were detected. The potential content of PCBs and toxic metals that may sorb onto the IX resins was conservatively estimated based on the COCs being present in basin water at reported detection limits. The calculations using the mass of the entire IXM showed that PCB and metal concentrations were less than TC screening levels with the exception of selenium, which had a calculated concentration of $41 \mathrm{mg} / \mathrm{kg}$ versus a screening level of $20 \mathrm{mg} / \mathrm{kg}$. Calculations using the total mass of the IXM and metal and PCB concentration in water estimates indicate the IXM may designate as hazardous waste for TC metals and TSCA regulated for PCBs. Calculations are provided in Appendix $C$. These calculations are based on a conservative approach, the IXM if designated as hazardous waste would be subject to treatment to meet LDR. The project is working to resolve the designation of the IXM.

\subsection{EXISTING REFERENCES}

Table 1-1 presents a list of the references that were reviewed as part of the scoping process, as well as a summary of the pertinent information contained within each reference. These references were the primary source for the background information presented in Section 1.4. 
Table 1-1. Existing References. (2 pages)

\begin{tabular}{|c|c|}
\hline Rerorence & summary \\
\hline $\begin{array}{l}\text { "Remove Debris from Storage Basin } \\
\text { for Disposal," OP-07-071, 8/17/1994. }\end{array}$ & $\begin{array}{l}\text { Procedure for Removal of Debris from K Basins. Describes } \\
\text { pressure washing, draining, obtaining dose rates upon } \\
\text { removal, and packaging for disposal. }\end{array}$ \\
\hline $\begin{array}{l}\text { "Facility Source Term Report," 99- } \\
\text { SNF/CJS-024, 3/29/99. }\end{array}$ & Provides 105 KE Smear Data. \\
\hline $\begin{array}{l}\text { "NDA Results for } 20 \text { Super } \\
\text { Compacted Drums at Allied } \\
\text { Technology Group," Summary Report } \\
\text { by Benchmark Environmental Corp. } \\
7 / 20 / 99 \text {. }\end{array}$ & $\begin{array}{l}\text { Provides NDA Results of } 20 \text { super-compacted drums from } \\
\text { K Basins. }\end{array}$ \\
\hline $\begin{array}{l}\text { "Categorizing and Inventorying Waste } \\
\text { in Standard Containers," Engineering } \\
\text { Data Transmittal } 619217,10 / 11 / 96 .\end{array}$ & $\begin{array}{l}\text { Provides dose rate to curie conversion factor of } 4^{\prime} \times 4^{\prime} \times 8^{\prime} \\
\text { wood box. }\end{array}$ \\
\hline $\begin{array}{l}\text { "Waste Certification Summary, lon } \\
\text { Exchange Modules," } 12 / 20 / 96 \text {. }\end{array}$ & $\begin{array}{l}\text { Table } 1 \text { provides "Historically Highest Low-Level IXM } \\
\text { Radionuclide Characterization." Document describes waste } \\
\text { generating process description, packaging, radiological } \\
\text { characterization, chemical characterization, waste } \\
\text { designation. }\end{array}$ \\
\hline $\begin{array}{l}\text { "Characterization Plan for Spent KE } \\
\text { Basin lon Exchange Modules," HNF- } \\
\text { SD-SNF-TI-039, Rev } 1 .\end{array}$ & $\begin{array}{l}\text { Provides basis for characterization, KE Basin isotopic ratios, } \\
\text { KE Basin Plutonium Ratios, Conversion Factors, sample } \\
\text { data. }\end{array}$ \\
\hline $\begin{array}{l}\text { "Ion Exchange Module High Integrity } \\
\text { Container Evaluation," memo from } \\
\text { Generator Services } 87610-95-033 \text {, } \\
4 / 28 / 95 \text {. }\end{array}$ & $\begin{array}{l}\text { Evaluation of IXM container found to meet the requirements } \\
\text { for acceptance as a HIC. }\end{array}$ \\
\hline $\begin{array}{l}\text { "Analytical Report for K Basin Paint," } \\
\text { FT-6112, 9/11/96. }\end{array}$ & $\begin{array}{l}\text { Analytical report consisting of TC metals for } 9 \text { paint samples } \\
\text { taken in K-Basin area. }\end{array}$ \\
\hline $\begin{array}{l}\text { "Analytical Report for K Basin Crane } \\
\text { Removal," Rev. 1, FD1-7021, 8/5/97. }\end{array}$ & $\begin{array}{l}\text { Analytical report consisting of ICP metals, Flashpoint, } \\
\text { PCBs, Total Alpha/Beta, Total Halides from } 11 \text { samples } \\
\text { taken from various points on a crane being removed from K- } \\
\text { Basin area. }\end{array}$ \\
\hline $\begin{array}{l}\text { "105 K East Basin Polychlorinated } \\
\text { Biphenyls Spill Cleanup Plan," } \\
\text { DOE/RL-96-53, Rev.0 (WHC 1996c) }\end{array}$ & $\begin{array}{l}\text { Description of KE Basin Sludge; sludge core samples } \\
\text { obtained from } 15 \text { locations in main basin, } 5 \text { in weasel pit; } \\
6 \text { out of } 20 \text { samples analyzed for PCBs in solids, } 2 \text { out of } 20 \\
\text { analyzed for PCBs in water phase. }\end{array}$ \\
\hline $\begin{array}{l}\text { "105 KE Basin PCB Wipe Sampling } \\
\text { and Analysis," WHC-SD-SNF-EV- } \\
001,3 / 28 / 96 \text {. (WHC 1996d) }\end{array}$ & $\begin{array}{l}\text { PCB Analysis performed on smear samples conducted on } \\
10 \text { canisters cleaned using Canister Cleaning System } \\
\text { procedure. Results indicate PCBs not detectable at } 0.1 \\
\text { microgram level. }\end{array}$ \\
\hline $\begin{array}{l}\text { 222-S Analytical Results for Process } \\
\text { water from KE and KW Fuel Basin, } \\
\text { 1/9/96. }\end{array}$ & $\begin{array}{l}\text { Analyses for water in K Basins : ICP metals, GEA, Sr-90, } \\
\text { H-3, Am-241, Alpha, Pu238/239, No PCBs detected. }\end{array}$ \\
\hline $\begin{array}{l}\text { "222-S Final Hanger Coupon Analysis } \\
\text { and Rad Survey Reports," } 1 / 30 / 97 .\end{array}$ & $\begin{array}{l}\text { Radiological characterization analyses on 3-pipe samples } \\
\text { taken from } 3 \text { fuel storage hangers from } 105 \mathrm{KE} \text {. }\end{array}$ \\
\hline $\begin{array}{l}\text { "Analytical Report for K Basin Pipe," } \\
\text { FT-6021, 6/5/99. }\end{array}$ & $\begin{array}{l}3 \text { pipe samples analyzed for TC metals, Am-241, Cs-137, } \\
\text { Pu-239/240, Sr-90, Total Uranium, GEA. }\end{array}$ \\
\hline $\begin{array}{l}\text { "Analytical Report for KE Pipes," } \\
\text { FD1-7002, 4/4/97. }\end{array}$ & $\begin{array}{l}2 \text { pipe samples analyzed for Am-241, Cs-137, Pu-239/240, } \\
\text { Sr-90, Total Uranium, GEA. }\end{array}$ \\
\hline
\end{tabular}


Table 1-1. Existing References. (2 pages)

\begin{tabular}{|c|c|}
\hline Reforonce & summary \\
\hline $\begin{array}{l}\text { "NDA Results for Waste Boxes and } \\
\text { Drums at } 100 \mathrm{~K} \text { Rad Pad," Battelle, } \\
7 / 12 / / 99 .\end{array}$ & $\begin{array}{l}\text { NDA (GEA) measurements conducted on eleven } 55 \text { gallon } \\
\text { drums and } 9 \text { waste boxes. }\end{array}$ \\
\hline $\begin{array}{l}\text { "Characterization of Empty Fuel } \\
\text { Storage Canisters in } 105 \text { KE Basin," } \\
\text { WHC-SD-SNF-TI-019, 6/27/96. }\end{array}$ & $\begin{array}{l}\text { In situ measurement of } 11 \text { canisters at KE Basin and } 40 \\
\text { swipe samples taken from } 9 \text { canisters upon removal. } \\
\text { Purpose of sampling to quantify isotopes contributing } \\
\text { significantly to overall activity on the canister. Describes the } \\
\text { cleaning process and radiological characterization of } \\
\text { residual isotopes. }\end{array}$ \\
\hline $\begin{array}{l}\text { BHI Archive Number } 0161426 . \\
\text { "105-N Basin Deactivation Project } \\
\text { Radioactive Waste Management } \\
\text { Archive, Volumes } 1 \text { and 2". July, } \\
\text { 1998. (BHI 1998). }\end{array}$ & $\begin{array}{l}\text { Compilation of data used to characterize N-reactor fuel and } \\
\text { fuel storage basin. Source data were applied to K-basin } \\
\text { waste to estimate radionuclide content of the waste and } \\
\text { provide process information. }\end{array}$ \\
\hline $\begin{array}{l}\text { WHC-SD-TP-SEP-028, Rev } 0 . \\
\text { "Safety Evaluation for Packaging the } \\
\text { N Reactor/Single Pass Reactor Fuel } \\
\text { Characterization Shipments", Oct. } \\
\text { 1994. (WHC 1994) }\end{array}$ & $\begin{array}{l}\text { A source of predicted isotopic composition of N-reactor fuel. } \\
\text { Used to assist in estimation of the radionuclide content of } \\
\text { the waste. }\end{array}$ \\
\hline $\begin{array}{l}\text { WHC-SD-NR-RPT-005, Rev } 0 . \\
\text { "Characterization of Radioactive } \\
\text { Waste at } 100 \text { Area", Nov. } 1990 \\
\text { (DeVanney 1990). }\end{array}$ & $\begin{array}{l}\text { Report contains analyses of samples from the KE and KW } \\
\text { Basin areas. These data provided analytical results used to } \\
\text { assist in the determination of contaminants of potential } \\
\text { concern (COPCs) and estimate of several radionuclides that } \\
\text { had not been estimated from other sources. }\end{array}$ \\
\hline $\begin{array}{l}\text { WHC-0063-4, Rev. 4, "Hanford Site } \\
\text { Solid Waste Acceptance Criteria," } \\
\text { June } 1998 .\end{array}$ & $\begin{array}{l}\text { Appendix } \mathrm{K}_{1} \text { Table K-1 is a list of radionuclides that would } \\
\text { be predicted in } \mathrm{N} \text {-Reactor fuel. Used as one source of } \\
\text { radionuclide ratios to estimate hard to measure } \\
\text { radionuclides (such as } \mathrm{H}-3 \text { and } \mathrm{Sm}-151 \text { ). }\end{array}$ \\
\hline
\end{tabular}

\subsection{DQO TEAM MEMBERS AND KEY DECISION MAKERS}

Table 1-2. DQO Team Members. (2 pages)

\begin{tabular}{|l|l|l|l|}
\hline \multicolumn{1}{|c|}{$\begin{array}{c}\text { Namo } \\
\text { Narganlation }\end{array}$} & \multicolumn{1}{c|}{$\begin{array}{c}\text { Aroa of Expertise } \\
\text { Telophone } \\
\text { Number }\end{array}$} \\
\hline Jeff Westcott & FH-WMP & Waste Management & $373-9800$ \\
\hline Bill Klover & WMTS & Rad Waste Analysis & $376-5082$ \\
\hline Dale Splett & DOE-RL & DOE SFO K Basins & $373-7827$ \\
\hline Jim Zimmerman & FDH & Nonrad Waste Designation & $373-3288$ \\
\hline Larry Oates & EQM & $\begin{array}{l}\text { Regulatory Support/ } \\
\text { Facilitator }\end{array}$ & $588-5529$ \\
\hline Paul Gagnon & WMFS-ERDF & ERDF Waste Management & $373-4379$ \\
\hline Paul Day & SNF Project & Regulatory Support K Basin & $376-4827$ \\
\hline
\end{tabular}


HNF-6273

Rev. 0

Table 1-2. DQO Team Members. (2 pages)

\begin{tabular}{|l|l|l|l|}
\hline \multicolumn{1}{|c|}{ Name } & \multicolumn{1}{c|}{ Organization } & \multicolumn{1}{c|}{ Area of Expertise } & \multicolumn{1}{c|}{$\begin{array}{c}\text { Tolephone } \\
\text { Number }\end{array}$} \\
\hline Wendy Thompson & BHI & BHI Sampling and Analysis & $372-9597$ \\
\hline Chuck Miller & EQM & Chemical Technical Support & $946-4985$ \\
\hline Mitzi Miller & EQM & Facilitator & $946-4985$ \\
\hline
\end{tabular}

Table 1-3. DQO Key Decision Makers.

\begin{tabular}{|l|l|l|l|}
\hline \multicolumn{1}{|c|}{ Name } & \multicolumn{1}{c|}{\begin{tabular}{c} 
Organization \\
\multicolumn{1}{|c|}{ Area of Expertise }
\end{tabular}} & \multicolumn{1}{c|}{$\begin{array}{c}\text { Telephone } \\
\text { Number }\end{array}$} \\
\hline Oscar M. Holgado & DOE-RL & DOE SFO Decision Maker & $373-0589$ \\
\hline Dave Einan & EPA & ERDF/EPA Decision Maker & $376-3883$ \\
\hline Larry Gadbois & EPA & EPAK-Basin Decision Maker & $376-9884$ \\
\hline Randy Jackson & BHI & $\begin{array}{l}\text { BHI Waste Designation } \\
\text { Represents BHI Decision Maker }\end{array}$ & $373-5473$ \\
\hline Chris Lucas & SNF-OPS & K-Basin Waste Generator & $373-1006$ \\
\hline David Watson & FDH & FDH/SNF Environmental Protection & $373-3250$ \\
\hline
\end{tabular}

\subsection{CONTAMINANTS OF POTENTIAL CONCERN}

\subsubsection{Master List of Contaminants of Potential Concern}

Table 1-4 identifies the individual components or waste media (e.g., piping, pumps, motors, or other facility-specific equipment) that are expected to be generated. The corresponding list of COPCs is identified for each WS.

\subsubsection{Excluded Contaminants of Potential Concern}

Table 1-5 lists the COPCs excluded from the investigation and the rationale for the exclusion.

\subsubsection{Final Contaminants of Concern List}

Table 1-6 provides the final list of COCs for each WS, with the rationale for inclusion.

The logic and tables discussing the radionuclides/isotopes remaining are presented in Appendix B, Table B-2. The COCs so derived are applicable to K-Basin waste that is below the ERDF WAC limits for cesium-137. If waste containing cesium-137 at levels greater than the ERDF WAC (BHI, 1998) encountered, then the COC selection criteria should be reevaluated prior to characterization and disposal. 
HNF-6273

Rev. 0

\subsection{PRELIMINARY ACTION LEVELS}

The preliminary actions levels that apply to each of the COCs are presented in Table 1-7 with the basis for the levels. The action level is defined as the threshold value that provides the criterion for choosing between alternative actions. The action levels presented in Table 1-7 are based on regulatory thresholds or standards and/or risk. The final numerical action level will be set in DQO Step 5 .

\subsection{STATEMENT OF THE PROBLEM}

Debris has been broadly defined by the K-Basin ROD (EPA et al. 1999) as all solid waste generated from the CERCLA interim remedial action of KE and KW Basins excluding SNF, sludge, and water. The debris has been previously disposed at the Hanford LLBG or CWC. This debris must be characterized and designated to allow disposal at ERDF, as appropriate. Because the K-Basin structures have been designated as an radioactive materials area, all materials removed from this area are assumed to be radioactively-contaminated. Most debris will designate as radioactive LLW, although some may designate as radioactive mixed waste or TRU. 
Table 1-4. Master List of COPCs for Each Component or Waste Stream. (4 pages)

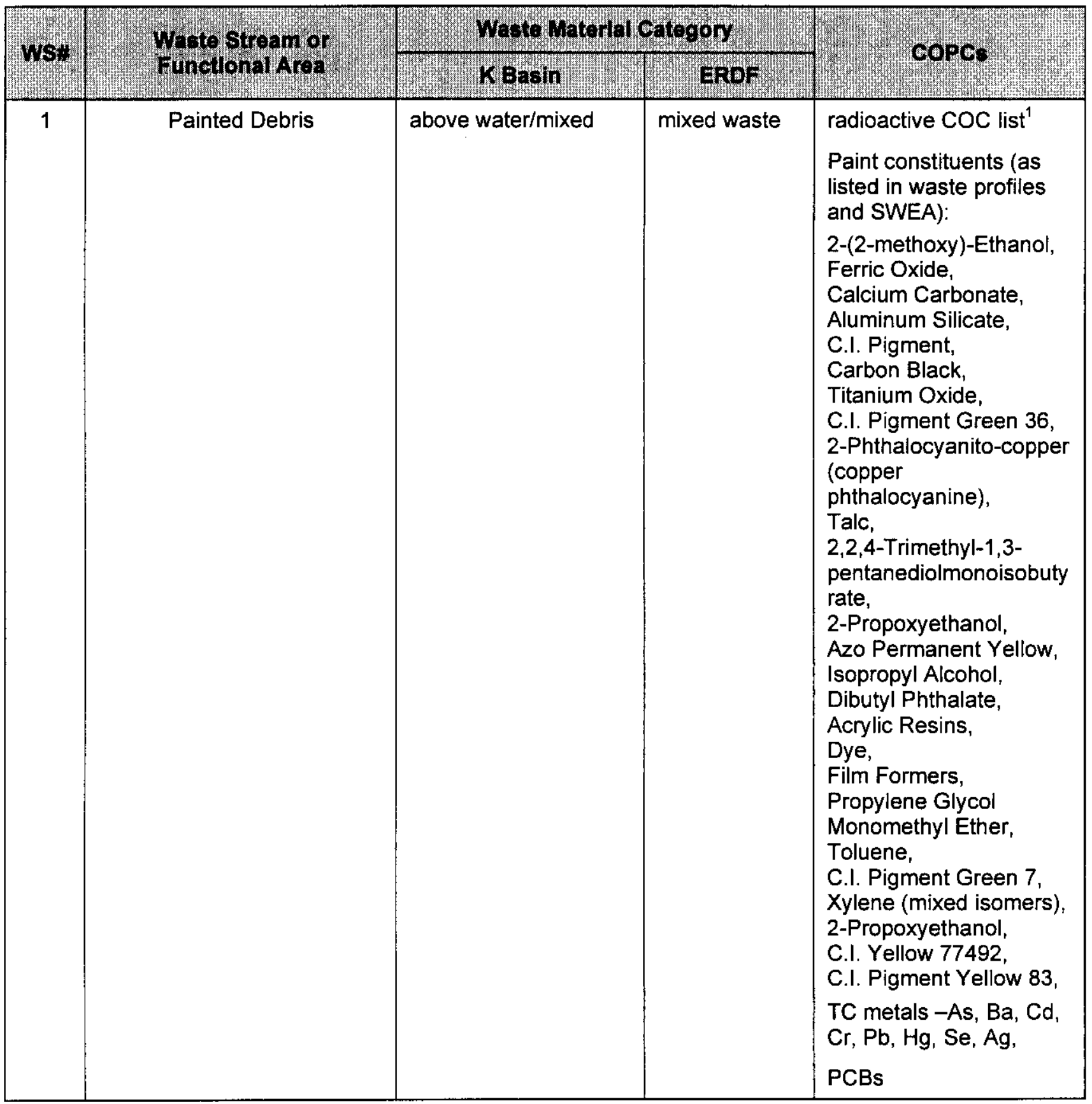


HNF-6273

Rev. 0

Table 1-4. Master List of COPCs for Each Component or Waste Stream. (4 pages)

\begin{tabular}{|c|c|c|c|c|}
\hline \multirow{2}{*}{ Wsy } & \multirow{2}{*}{$\begin{array}{l}\text { Weste strodm or } \\
\text { Functional Aroa }\end{array}$} & \multicolumn{2}{|c|}{ Wasta Maternal category } & \multirow{2}{*}{ copcs } \\
\hline & & K Basin & ERDF & \\
\hline 2 & $\begin{array}{l}\text { Rags Contaminated with } \\
\text { Stripped Paint Waste } \\
\text { (Citristrip) }\end{array}$ & above water/mixed & mixed waste & $\begin{array}{l}\text { radioactive COC list }^{1} \\
\mathrm{TC} \text { metals -As, Ba, Cd, } \\
\mathrm{Cr}, \mathrm{Pb}, \mathrm{Hg}, \mathrm{Se}, \mathrm{Ag} \\
\text { Paint constituents from } \\
\text { SWEA/waste profile: } \\
\text { Stoddard Solvent } \\
\text { N-Butyl Acetate } \\
\text { Naphthalene } \\
\text { Ethyl Benzene } \\
\text { Aluminum Phosphate } \\
\text { monobasic } \\
\text { Hydroxypropylmethyl- } \\
\text { cellulose } \\
\text { Citristrip constituents } \\
\text { from SWEA/waste } \\
\text { profile: } \\
\mathrm{N} \text {-Methyl-2-pyrrolidone } \\
\text { D-Limonene } \\
\text { PCBs }\end{array}$ \\
\hline 3 & $\begin{array}{l}\text { structural shielding that } \\
\text { contains haz metals - lead } \\
\text { bricks, lead shielding }\end{array}$ & $\begin{array}{l}\text { above water/ mixed } \\
\text { waste }\end{array}$ & mixed waste & $\begin{array}{l}\mathrm{Pb} \\
\text { Radioactive COC } \text { list }^{1}\end{array}$ \\
\hline 4 & $\begin{array}{c}\text { Broken Fluorescent and } \\
\text { incandescent light bulbs } \\
\text { (ballasts/fixture assumed not } \\
\text { present in the basin) }\end{array}$ & $\begin{array}{l}\text { above water/ mixed } \\
\text { waste }\end{array}$ & mixed waste & $\begin{array}{l}\mathrm{Hg}, \mathrm{Pb} \\
\text { Radioactive COC list }{ }^{1}\end{array}$ \\
\hline 5 & $\begin{array}{l}\text { cartridge filters, disposable } \\
\text { PPE, plastic, and other trash }\end{array}$ & above water/ LLW & $\begin{array}{l}\text { Low-Level } \\
\text { Rad Waste } \\
\end{array}$ & radioactive COC list ${ }^{1}$ \\
\hline 6 & $\begin{array}{l}\text { materials used for decon of } \\
\text { equipment: cloth, paper, } \\
\text { plastic }\end{array}$ & above water/ LLW & $\begin{array}{l}\text { Low-Level } \\
\text { Rad Waste }\end{array}$ & radioactive COC list $^{1}$ \\
\hline 7 & $\begin{array}{l}\text { process equipment: heat } \\
\text { exchangers, piping }\end{array}$ & above water/ LLW & $\begin{array}{l}\text { Low-Level } \\
\text { Rad Waste }\end{array}$ & $\begin{array}{l}\text { radioactive COC list }{ }^{1} \\
\text { lead }\end{array}$ \\
\hline 8 & $\begin{array}{l}\text { Unpainted demolition debris, } \\
\text { structural steel, rocks, } \\
\text { gravel, metal, glass, } \\
\text { concrete, ceramic, bricks, } \\
\text { roofing material, wood } \\
\text { drywall, siding }\end{array}$ & above water/ LLW & $\begin{array}{l}\text { Low-Level } \\
\text { Rad Waste }\end{array}$ & $\begin{array}{l}\text { radioactive COC list } \\
\text { asbestos }\end{array}$ \\
\hline
\end{tabular}


HNF-6273

Rev. 0

Table 1-4. Master List of COPCs for Each Component or Waste Stream. (4 pages)

\begin{tabular}{|c|c|c|c|c|}
\hline \multirow{2}{*}{ WSH } & \multirow{2}{*}{ Wunctoner Area } & \multicolumn{2}{|c|}{ Waste Material Category } & \multirow{2}{*}{ coras } \\
\hline & & KEFsin & ERDF & \\
\hline 9 & $\begin{array}{l}\text { materials collected during } \\
\text { general housekeeping: soil, } \\
\text { sawdust, vegetation, debris, } \\
\text { glass, plastic }\end{array}$ & above water/ LLW & $\begin{array}{l}\text { Low-Level } \\
\text { Rad Waste }\end{array}$ & radioactive COC list ${ }^{1}$ \\
\hline 10 & HEPA filters & above water/ LLW & $\begin{array}{l}\text { Low-Level } \\
\text { Rad Waste }\end{array}$ & radioactive COC list ${ }^{1}$ \\
\hline 11 & $\begin{array}{l}\text { structural steel - fuel } \\
\text { storage racks \& bulkheads; } \\
\text { structures used for fuel } \\
\text { handling }\end{array}$ & $\begin{array}{l}\text { underwater/ } \\
\text { LLW or mixed }\end{array}$ & $\begin{array}{l}\text { Low-Level } \\
\text { Rad Waste or } \\
\text { mixed }\end{array}$ & 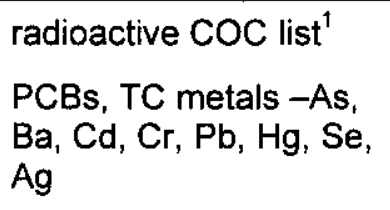 \\
\hline 12 & $\begin{array}{c}\text { process equipment - } \\
\text { pumps, old canister washer, } \\
\text { piping and piping } \\
\text { components, rubber hoses }\end{array}$ & $\begin{array}{l}\text { underwaterl } \\
\text { LLW or mixed }^{2}\end{array}$ & $\begin{array}{l}\text { Low-Level } \\
\text { Rad Waste or } \\
\text { mixed }\end{array}$ & 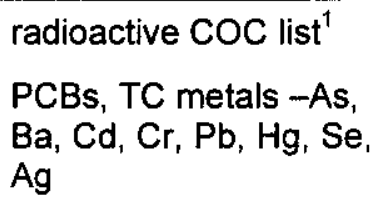 \\
\hline 13 & $\begin{array}{l}\text { miscellaneous debris - } \\
\text { electrical cables, light } \\
\text { fixtures, long tools, brushes, } \\
\text { PPE, metal, plastic }\end{array}$ & $\begin{array}{l}\text { underwater/ } \\
\text { LLW or mixed }{ }^{2}\end{array}$ & $\begin{array}{l}\text { Low-Level } \\
\text { Rad Waste or } \\
\text { mixed }\end{array}$ & 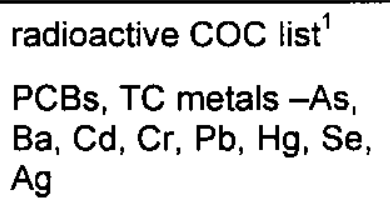 \\
\hline 14 & Canisters/canister lids & $\begin{array}{l}\text { underwater/ } \\
\text { LLW or mixed }{ }^{2}\end{array}$ & $\begin{array}{l}\text { Low-Level } \\
\text { Rad Waste or } \\
\text { mixed }\end{array}$ & $\begin{array}{l}{\text { radioactive COC } \text { list }^{1}} \\
\mathrm{PCBs}, \mathrm{TC} \text { metals }-\mathrm{As} \text {, } \\
\mathrm{Ba}, \mathrm{Cd}, \mathrm{Cr}, \mathrm{Pb}, \mathrm{Hg}, \mathrm{Se} \text {, } \\
\mathrm{Ag}\end{array}$ \\
\hline 15 & IXMs & $\begin{array}{l}\text { above water/ } \\
\text { LLW or mixed }^{2}\end{array}$ & $\begin{array}{l}\text { Low-Level } \\
\text { Rad Waste or } \\
\text { mixed }\end{array}$ & $\begin{array}{l}\text { radioactive COC list }{ }^{1} \\
\mathrm{PCBs}, \mathrm{TC} \text { metals }-\mathrm{As} \text {, } \\
\mathrm{Ba}, \mathrm{Cd}, \mathrm{Cr}, \mathrm{Pb}, \mathrm{Hg}, \mathrm{Se} \text {, } \\
\mathrm{Ag}\end{array}$ \\
\hline 16 & $\begin{array}{l}\text { floor tiles/ceiling tiles; } \\
\text { sprayed on ceiling texture or } \\
\text { acoustic surface coatings }\end{array}$ & $\begin{array}{l}\text { above water/ } \\
\text { LLW or mixed }^{3}\end{array}$ & $\begin{array}{l}\text { Low-Level } \\
\text { Rad Waste or } \\
\text { mixed }\end{array}$ & $\begin{array}{l}\mathrm{Pb}, \mathrm{PCBs} \text {, and organics } \\
\text { from } \mathrm{WS} 1 \text {, if painted } \\
\text { asbestos } \\
\text { radioactive COC list }{ }^{1}\end{array}$ \\
\hline
\end{tabular}


HNF-6273

Rev. 0

Table 1-4. Master List of COPCs for Each Component or Waste Stream. (4 pages)

\begin{tabular}{|c|c|c|c|c|}
\hline \multirow{2}{*}{ wsm } & \multirow{2}{*}{ w whet stream or } & \multicolumn{2}{|c|}{ Whet Wateria catogory } & \multirow{2}{*}{ apros } \\
\hline & & K Kasin & EñF & \\
\hline 17 & $\begin{array}{l}\text { pipe and duct insulation and } \\
\text { insulation mastic; mastic } \\
\text { used as adhesive for plastic } \\
\text { baseboard moldings }\end{array}$ & $\begin{array}{l}\text { above water/ } \\
\text { LLW } \\
\text { Asbestos }\end{array}$ & $\begin{array}{l}\text { Low-Level } \\
\text { Rad Waste }\end{array}$ & $\begin{array}{l}\text { asbestos } \\
\text { radioactive COC list }{ }^{1}\end{array}$ \\
\hline 18 & $\begin{array}{c}\text { mineral based building } \\
\text { insulation in walls and } \\
\text { ceilings }\end{array}$ & $\begin{array}{l}\text { above water/ } \\
\text { LLW } \\
\text { asbestos }\end{array}$ & $\begin{array}{l}\text { Low-Level } \\
\text { Rad Waste }\end{array}$ & $\begin{array}{l}\text { asbestos } \\
\text { radioactive COC } \text { list }^{1}\end{array}$ \\
\hline 19 & $\begin{array}{l}\text { asbestos board (transite) } \\
\text { used in walls, ceilings, } \\
\text { siding }\end{array}$ & $\begin{array}{l}\text { above water/ } \\
\text { LLW } \\
\text { asbestos }\end{array}$ & $\begin{array}{l}\text { Low-Level } \\
\text { Rad Waste }\end{array}$ & $\begin{array}{l}\text { asbestos } \\
\text { radioactive COC } \text { list }^{1}\end{array}$ \\
\hline 20 & high temp gaskets and seals & $\begin{array}{l}\text { above water/ } \\
\text { LLW } \\
\text { PCBs } \\
\text { asbestos }\end{array}$ & $\begin{array}{l}\text { Low-Level } \\
\text { Rad Waste }\end{array}$ & $\begin{array}{l}\text { asbestos } \\
\text { radioactive COC } \text { list }^{1}\end{array}$ \\
\hline
\end{tabular}

${ }^{1}$ Radiological COPCs are numerous (80) and are provided in Appendix B, Table B-1.

${ }^{2}$ Radioactive/LLW could potentially designate as mixed waste if the sludge is incompletely removed, or if the underwater debris items are porous.

${ }^{3} \mathrm{TC}$ Metals in paint may cause this Radioactive/LLW to be designated as mixed waste.

Table 1-5. Rationale for COPC Exclusions. (3 pages)

\begin{tabular}{|c|c|c|c|c|}
\hline Ws & Waste stroam or & (component) & 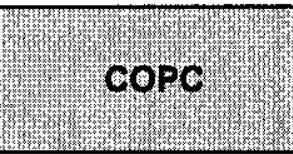 & $\begin{array}{l}\text { Rationalo for } \\
\text { Exciusion }\end{array}$ \\
\hline \multirow[t]{6}{*}{1} & \multirow[t]{6}{*}{ Painted Debris } & \multirow[t]{6}{*}{$\begin{array}{l}\text { above water/ } \\
\text { LLW or mixed }\end{array}$} & $\begin{array}{l}\text { radioactive COC } \\
\text { list }^{1}\end{array}$ & see Appendix B \\
\hline & & & $\begin{array}{l}\text { Paint } \\
\text { Constituents: }\end{array}$ & \\
\hline & & & $\begin{array}{l}\text { 2-(2-methoxy)- } \\
\text { Ethanol }\end{array}$ & $\begin{array}{l}\text { Volatile/ vp>1mm Hg } \\
\text { at } 20 \text { degrees } \mathrm{C}^{3}\end{array}$ \\
\hline & & & Ferric Oxide & Not Toxic \\
\hline & & & $\begin{array}{l}\text { Calcium } \\
\text { Carbonate }\end{array}$ & Not Toxic \\
\hline & & & Aluminum Silicate & Not Toxic \\
\hline
\end{tabular}


HNF-6273

Rev. 0

Table 1-5. Rationale for COPC Exclusions. (3 pages)

\begin{tabular}{|c|c|c|c|c|}
\hline ws & $\begin{array}{l}\text { Waste stream or } \\
\text { Functionat Area }\end{array}$ & (Componont) & cope & $\begin{array}{l}\text { Rationale or } \\
\text { Exclusion }\end{array}$ \\
\hline & & & C.I. Pigment & content not identified \\
\hline & & & Carbon Black & Not Toxic \\
\hline & & & Titanium Oxide & Not Toxic \\
\hline & & & $\begin{array}{l}\text { C.I. Pigment } \\
\text { Green } 36\end{array}$ & content not identified \\
\hline & & & Talc & Not Toxic \\
\hline & & & $\begin{array}{l}\text { Azo Permanent } \\
\text { Yellow }\end{array}$ & content not identified \\
\hline & & & Isopropyl Alcohol & $\begin{array}{l}\text { Volatile/ } \mathrm{vp}>1 \mathrm{mmm}^{3} \mathrm{Hg} \\
\text { at } 20 \text { degrees } \mathrm{C}^{3}\end{array}$ \\
\hline & & & acrylic resins & Not Toxic \\
\hline & & & Dye & content not identified \\
\hline & & & Film Formers & content not identified \\
\hline & & & $\begin{array}{l}\text { Propylene Gycol } \\
\text { Monomethyl Ether }\end{array}$ & $\begin{array}{l}\text { Volatile/ } \mathrm{vp}>1 \mathrm{mmm}^{3} \mathrm{Hg} \\
\text { at } 20 \text { degrees } \mathrm{C}^{3}\end{array}$ \\
\hline & & & Toluene & $\begin{array}{l}\text { Volatile/ vp }>1 \mathrm{mmm}^{3} \mathrm{Hg} \\
\text { at } 20 \text { degrees } \mathrm{C}^{3}\end{array}$ \\
\hline & & & $\begin{array}{l}\text { C.I. Pigment } \\
\text { Green } 7\end{array}$ & content not identified \\
\hline & & & Xylene & $\begin{array}{l}\text { Volatile/ vp }>1 \mathrm{mmH}^{3} \mathrm{gg} \\
\text { at } 20 \text { degrees } \mathrm{C}^{3}\end{array}$ \\
\hline & & & 2-Propoxyethanol & $\begin{array}{l}\text { Volatile/ } \mathrm{vp}>1 \mathrm{mmm}^{3} \mathrm{Hg} \\
\text { at } 20 \text { degrees } \mathrm{C}^{3}\end{array}$ \\
\hline & & & C.I. Yellow 77492 & content not identified \\
\hline & & & $\begin{array}{l}\text { C.I. Pigment } \\
\text { Yellow } 83\end{array}$ & content not identified \\
\hline & & & PCBs & $\begin{array}{l}\text { Excluded for paint } \\
\text { waste disposal at } \\
\text { ERDF }\end{array}$ \\
\hline \multirow[t]{3}{*}{2} & \multirow{3}{*}{$\begin{array}{l}\text { Rags Contaminated with } \\
\text { Stripped Paint Waste } \\
\text { (Citristrip) }\end{array}$} & \multirow[t]{3}{*}{$\begin{array}{l}\text { above water/ } \\
\text { mixed }\end{array}$} & $\begin{array}{l}\text { radioactive COC } \\
\text { list }^{1}\end{array}$ & See Appendix B \\
\hline & & & Paint constituents: & \\
\hline & & & Stoddard Solvent & $\begin{array}{l}\text { Volatile/ vp>1 } \mathrm{mmHg}^{3} \mathrm{Hg} \\
\text { at } 20 \text { degrees } \mathrm{C}^{3}\end{array}$ \\
\hline
\end{tabular}


HNF-6273

Rev. 0

Table 1-5. Rationale for COPC Exclusions. (3 pages)

\begin{tabular}{|c|c|c|c|c|}
\hline ws & Hpere strean or & $\begin{array}{l}\text { Materal } \\
\text { component) }\end{array}$ & copc & $\begin{array}{l}\text { Rationale ror } \\
\text { Exclusion }\end{array}$ \\
\hline & & & N-Butyl Acetate & $\begin{array}{l}\text { Volatile/ vp> } 1 \mathrm{mmHg}^{3} \\
\text { at } 20 \text { degrees } \mathrm{C}^{3}\end{array}$ \\
\hline & & & Ethyl Benzene & $\begin{array}{l}\text { Volatile/ vp>1 } \mathrm{mmH}^{3} \mathrm{Hg} \\
\text { at } 20 \text { degrees } \mathrm{C}^{3}\end{array}$ \\
\hline & & & $\begin{array}{l}\text { Aluminum } \\
\text { Phosphate } \\
\text { monobasic }\end{array}$ & Not Toxic \\
\hline
\end{tabular}

${ }^{1}$ Radiological COPCs are numerous (80) and are provided in Appendix B, Table B-1. Rationale for Exclusion is also provided in Appendix B.

${ }^{2} \mathrm{TC}$ Metals in paint may cause this Radioactive/LLW to be designated as mixed waste.

${ }^{3}$ Volatile constituents of paint, although unlikely to be present in dried paint, must remain as a COPC until objective evidence has been obtained through analysis or examination of other dried paint with similar volatile content. Refer to Section 1.3.2 item 6.

Table 1-6. Final List of COCs. (5 pages)

\begin{tabular}{|c|c|c|c|}
\hline Hs: & $\begin{array}{c}\text { Matorial (Componthy) } \\
\text { Cotogory }\end{array}$ & (x) & Rationale for inclusion \\
\hline 1 & Painted Debris & $\begin{array}{l}\text { radioactive COC list } \\
\text { TC metals }-\mathrm{As}, \mathrm{Ba}, \mathrm{Cd} \text {, } \\
\mathrm{Cr}, \mathrm{Pb}, \mathrm{Hg}, \mathrm{Se}, \mathrm{Ag} \\
\text { 2-(2-methoxy)-Ethanol, 2- } \\
\text { Phthalocyanito-copper } \\
\text { (copper phthalocyanine), } \\
\text { 2,2,4-Trimethyl-1,3- } \\
\text { pentanediolmonoisobutyra } \\
\text { te, 2-propoxyethanol, } \\
\text { Dibutyl Phthalate, } \\
\text { Naphthalene, } \\
\text { Hydroxypropylmethylcellul } \\
\text { ose, }\end{array}$ & $\begin{array}{l}\text { Radioactive COC } \text { list }^{1} \\
\text { Metals confirmed to be present } \\
\text { in paint at concentrations above } \\
\text { screening limits for TC. } \\
\text { Nonvolatile paint constituents. } \\
\text { Toxicity must be evaluated to } \\
\text { determine the contribution to } \\
\text { Dangerous Waste Criteria } \\
\text { Equivalent Concentration per } \\
\text { Washington Administrative Code } \\
173-303-100 \\
\text { *NOTE: Volatile paint } \\
\text { constituents identified in Table } \\
1-5 \text { for exclusion cannot be } \\
\text { excluded without objective } \\
\text { evidence, see Section } 1.3 .2 \\
\text { item } 6 \text {. }\end{array}$ \\
\hline
\end{tabular}


HNF-6273

Rev. 0

Table 1-6. Final List of COCs. (5 pages)

\begin{tabular}{|c|c|c|c|}
\hline ws: & $\begin{array}{l}\text { Material (Component) } \\
\text { Catogory }\end{array}$ & coc & Rationale for inciusion \\
\hline 2 & $\begin{array}{l}\text { Rags Contaminated with } \\
\text { Stripped Paint Waste }\end{array}$ & $\begin{array}{l}\text { radioactive COC list } \\
{ }^{1} \\
\mathrm{TC} \text { metals -As, } \mathrm{Ba}, \mathrm{Cd}, \\
\mathrm{Cr}, \mathrm{Pb}, \mathrm{Hg}, \mathrm{Se}, \mathrm{Ag} \\
\text { 2-(2-methoxy)-Ethanol, 2- } \\
\text { Phthalocyanito-copper } \\
\text { (copper phthalocyanine), } \\
\text { 2,2,4-Trimethyl-1,3- } \\
\text { pentanediolmonoisobutyra } \\
\text { te, 2-propoxyethanol, } \\
\text { Dibutyl Phthalate, N- } \\
\text { Naphthalene, } \\
\text { Hydroxypropylmethyl- } \\
\text { cellulose } \\
\text { Methyl-2-pyrrolidone, D- } \\
\text { Limonene }\end{array}$ & $\begin{array}{l}\text { radioactive COC list } \\
\text { Metals confirmed to be present } \\
\text { in paint at concentrations above } \\
\text { screening limits for TC. } \\
\text { Nonvolatile paint constituents. } \\
\text { Toxicity must be evaluated to } \\
\text { determine the contribution to } \\
\text { Dangerous Waste Criteria } \\
\text { Equivalent Concentration per } \\
\text { Washington Administrative } \\
\text { Code173-303-100. } \\
\text { *NOTE: Volatile paint } \\
\text { constituents identified in Table } \\
1-5 \text { for exclusion cannot be } \\
\text { excluded without objective } \\
\text { evidence, see section } 1.3 .2 \\
\text { item } 6 . \\
\text { Citristrip constituents. Toxicity } \\
\text { must be evaluated to determine } \\
\text { the contribution to Dangerous } \\
\text { Waste Criteria Equivalent } \\
\text { Concentration per Washington } \\
\text { Administrative Code } 173-303- \\
100 \\
\text { NOTE: D-Limonene is a } \\
\text { Washington "Toxic D" waste if } \\
\text { present at } 10 \% \text { or greater. }\end{array}$ \\
\hline 3 & $\begin{array}{l}\text { structural shielding that } \\
\text { contains haz metals - lead } \\
\text { bricks, lead shielding }\end{array}$ & $\begin{array}{l}{\text { radioactive COC } \text { list }^{1}}^{\mathrm{Pb}}\end{array}$ & $\begin{array}{l}\text { radioactive COC list }{ }^{1} \\
\text { Major component in lead } \\
\text { shielding }\end{array}$ \\
\hline 4 & $\begin{array}{l}\text { Broken fluorescent and } \\
\text { incandescent light bulbs } \\
\text { (ballasts/fixture assumed not } \\
\text { present in the basin) }\end{array}$ & $\begin{array}{l}\text { radioactive COC list }^{1} \\
\mathrm{TC} \text { metals }-\mathrm{As}, \mathrm{Ba}, \mathrm{Cd} \text {, } \\
\mathrm{Cr}, \mathrm{Pb}, \mathrm{Hg}, \mathrm{Se}, \mathrm{Ag}\end{array}$ & $\begin{array}{l}\text { radioactive COC list } \\
\text { Metals present in fluorescent } \\
\text { and incandescent bulbs }\end{array}$ \\
\hline 5 & $\begin{array}{l}\text { cartridge filters, disposable } \\
\text { PPE, plastic, and other trash }\end{array}$ & radioactive COC list ${ }^{1}$ & 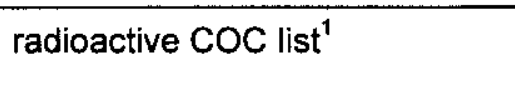 \\
\hline 6 & $\begin{array}{l}\text { materials used for decon of } \\
\text { equipment: cloth, paper, } \\
\text { plastic }\end{array}$ & radioactive COC list $^{1}$ & radioactive COC list $^{1}$ \\
\hline 7 & $\begin{array}{l}\text { process equipment: heat } \\
\text { exchangers, piping }\end{array}$ & 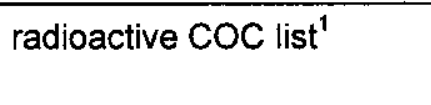 & radioactive COC list ${ }^{1}$ \\
\hline
\end{tabular}


HNF-6273

Rev. 0

Table 1-6. Final List of COCs. (5 pages)

\begin{tabular}{|c|c|c|c|}
\hline Wsy & 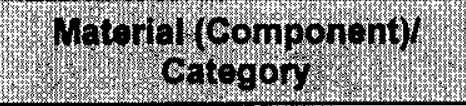 & coc & Rationalo for incliston \\
\hline 8 & $\begin{array}{l}\text { Unpainted demolition debris, } \\
\text { structural steel, rocks, } \\
\text { gravel, metal, glass, } \\
\text { concrete, ceramic, bricks, } \\
\text { roofing material, wood } \\
\text { drywall, siding }\end{array}$ & radioactive COC list ${ }^{1}$ & radioactive COC list ${ }^{1}$ \\
\hline 9 & $\begin{array}{l}\text { materials collected during } \\
\text { general housekeeping: soil, } \\
\text { sawdust, vegetation, debris, } \\
\text { glass, plastic }\end{array}$ & radioactive COC list ${ }^{1}$ & radioactive $\mathrm{COC}$ list $^{1}$ \\
\hline 10 & HEPA filters & radioactive COC list $^{1}$ & radioactive COC list $^{1}$ \\
\hline 11 & $\begin{array}{l}\text { structural steel - fuel } \\
\text { storage racks \& bulkheads; } \\
\text { structures used for fuel } \\
\text { handling }\end{array}$ & $\begin{array}{l}\text { radioactive COC list, } 2 \\
\mathrm{PCBs}, \mathrm{TC} \text { metals }-\mathrm{As}, \mathrm{Ba} \text {, } \\
\mathrm{Cd}, \mathrm{Cr}, \mathrm{Pb}, \mathrm{Hg}, \mathrm{Se}, \mathrm{Ag}\end{array}$ & $\begin{array}{l}\text { radioactive COC list }^{1} \\
\text { Metals and PCBs have been } \\
\text { identified in KE Basin Sludge at } \\
\text { concentrations exceeding the } \\
\text { TCLP Total Concentration } \\
\text { screening level. If sludge is } \\
\text { incompletely removed or if } \\
\text { underwater items are porous, } \\
\text { then the presence of residual } \\
\text { sludge may cause the items to } \\
\text { be designated as mixed waste. }\end{array}$ \\
\hline 12 & $\begin{array}{c}\text { process equipment - } \\
\text { pumps, old canister washer, } \\
\text { piping and piping } \\
\text { components, rubber hoses }\end{array}$ & $\begin{array}{l}\text { radioactive COC list }{ }^{1,2} \\
\mathrm{PCBs}, \mathrm{TC} \text { metals }-\mathrm{As}, \mathrm{Ba} \text {, } \\
\mathrm{Cd}, \mathrm{Cr}, \mathrm{Pb}, \mathrm{Hg}, \mathrm{Se}, \mathrm{Ag}\end{array}$ & $\begin{array}{l}\text { radioactive COC list }^{1} \\
\text { Metals and PCBs have been } \\
\text { identified in KE Basin Sludge at } \\
\text { concentrations exceeding the } \\
\text { TCLP Total Concentration } \\
\text { screening level. If sludge is } \\
\text { incompletely removed or if } \\
\text { underwater items are porous, } \\
\text { then the presence of residual } \\
\text { sludge may cause the items to } \\
\text { be designated as mixed waste. }\end{array}$ \\
\hline 13 & $\begin{array}{c}\text { miscellaneous debris - } \\
\text { electrical cables, light } \\
\text { fixtures, long tools, brushes, } \\
\text { PPE, metal, plastic }\end{array}$ & $\begin{array}{l}\text { radioactive } \mathrm{COC} \text { list }^{1,2} \\
\mathrm{PCBs}, \mathrm{TC} \text { metals }-\mathrm{As}, \mathrm{Ba} \text {, } \\
\mathrm{Cd}, \mathrm{Cr}, \mathrm{Pb}, \mathrm{Hg}, \mathrm{Se}, \mathrm{Ag}\end{array}$ & $\begin{array}{l}\text { radioactive COC list } \\
\text { Metals and PCBs have been } \\
\text { identified in KE Basin Sludge at } \\
\text { concentrations exceeding the } \\
\text { TCLP Total Concentration } \\
\text { screening level. If sludge is } \\
\text { incompletely removed or if } \\
\text { underwater items are porous, } \\
\text { then the presence of residual } \\
\text { sludge may cause the items to } \\
\text { be designated as mixed waste. }\end{array}$ \\
\hline
\end{tabular}


HNF-6273

Rev. 0

Table 1-6. Final List of COCs. (5 pages)

\begin{tabular}{|c|c|c|c|}
\hline$w s$ & $\begin{array}{c}\text { waterial (Conponent) } \\
\text { Cagory }\end{array}$ & cod & Rationale for incivion \\
\hline 14 & Canisters/canister lids & 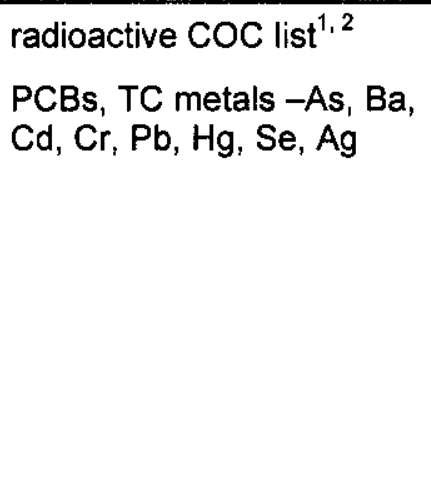 & $\begin{array}{l}\text { radioactive COC list' } \\
\text { Metals and PCBs have been } \\
\text { identified in KE Basin Sludge at } \\
\text { concentrations exceeding the } \\
\text { TCLP Total Concentration } \\
\text { screening level. If sludge is } \\
\text { incompletely removed or if } \\
\text { underwater items are porous, } \\
\text { then the presence of residual } \\
\text { sludge may cause the items to } \\
\text { be designated as mixed waste. }\end{array}$ \\
\hline 15 & IXMs & 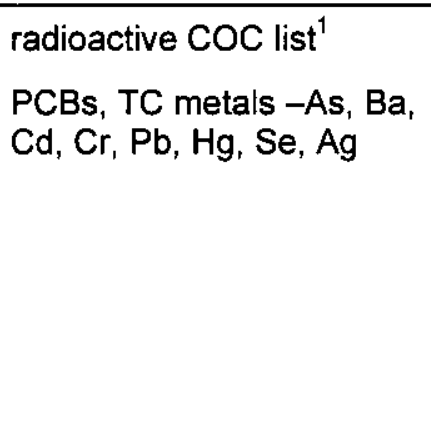 & $\begin{array}{l}\text { radioactive COC list } \\
\text { PCBs in water at concentrations } \\
\text { at or near the reported detection } \\
\text { limit may be expected to bind to } \\
\text { the hydrophobic IXM resin } \\
\text { material. Toxic Metals in water } \\
\text { at concentrations at or near the } \\
\text { detection limit may concentrate } \\
\text { to elevated concentrations in the } \\
\text { spent IXMs. }\end{array}$ \\
\hline 16 & $\begin{array}{l}\text { floor tiles/ceiling tiles; } \\
\text { sprayed on ceiling texture or } \\
\text { acoustic surface coatings }\end{array}$ & $\begin{array}{l}\mathrm{Pb} \text {, if painted } \\
\text { asbestos } \\
\text { radioactive COC list }{ }^{1}\end{array}$ & $\begin{array}{l}\text { ACM may be painted. If lead } \\
\text { paint is applied, } A C M \text { must } \\
\text { contain less than } 0.05 \% \text { wt. } \\
\text { paint. } \\
\text { The age of the KE and } \mathrm{KW} \\
\text { Basin facilities indicates that } \\
\text { asbestos is likely to be present } \\
\text { in numerous materials. } \\
\text { radioactive COC list }{ }^{1}\end{array}$ \\
\hline 17 & $\begin{array}{l}\text { pipe and duct insulation and } \\
\text { insulation mastic; mastic } \\
\text { used as adhesive for plastic } \\
\text { baseboard moldings }\end{array}$ & $\begin{array}{l}\text { asbestos } \\
\text { radioactive COC list }\end{array}$ & $\begin{array}{l}\text { The age of the KE and } \mathrm{KW} \\
\text { Basin facilities indicates that } \\
\text { asbestos is likely to be present } \\
\text { in numerous materials. } \\
\text { radioactive COC list }\end{array}$ \\
\hline 18 & $\begin{array}{c}\text { mineral based building } \\
\text { insulation in walls and } \\
\text { ceilings }\end{array}$ & $\begin{array}{l}\text { asbestos } \\
\text { radioactive COC list }{ }^{1}\end{array}$ & $\begin{array}{l}\text { The age of the KE and } \mathrm{KW} \\
\text { Basin facilities indicates that } \\
\text { asbestos is likely to be present } \\
\text { in numerous materials. } \\
\text { radioactive COC } \text { list }^{1}\end{array}$ \\
\hline
\end{tabular}


HNF-6273

Rev. 0

Table 1-6. Final List of COCs. (5 pages)

\begin{tabular}{|c|c|c|c|}
\hline Wsy & $\begin{array}{c}\text { Materal r component) } \\
\text { Catogory }\end{array}$ & coc & Rationale tor Inclusion \\
\hline 19 & $\begin{array}{l}\text { asbestos board (transite) } \\
\text { used in walls, ceilings, } \\
\text { siding }\end{array}$ & $\begin{array}{l}\text { asbestos } \\
\text { radioactive COC list }{ }^{1}\end{array}$ & $\begin{array}{l}\text { The age of the KE and } \mathrm{KW} \\
\text { Basin facilities indicates that } \\
\text { asbestos is likely to be present } \\
\text { in numerous materials. } \\
\text { radioactive COC list }{ }^{1}\end{array}$ \\
\hline 20 & high temp gaskets and seals & $\begin{array}{l}\text { asbestos } \\
\text { radioactive COC list }{ }^{1}\end{array}$ & $\begin{array}{l}\text { The age of the } \mathrm{KE} \text { and } \mathrm{KW} \\
\text { Basin facilities indicates that } \\
\text { asbestos is likely to be present } \\
\text { in numerous materials. } \\
\text { radioactive COC list }\end{array}$ \\
\hline
\end{tabular}

${ }^{1}$ Radiological COCs are H3, Co-60, Ni-63, Sr-90, Sb-125, Cs/Ba-137, Pm-147, Sm-151, Eu-152, Eu-154, Eu-155, U-235, U-238, Pu-238, Pu-239, Pu-240, Pu-241, Am-241, and Cm-244. Each radionuclide has been included because they meet one or more of the following criteria (1) the radionuclide is part of the $\mathrm{N}$-Reactor uranium fuel cycle process, (2) the radionuclide is not gaseous and has a half-life greater than 1 year, (3) the beta/gamma emitting radionuclide was estimated to be present at greater than $1 \%$ of the Cs-137 activity of the waste, and/or (4) the alpha emitting or TRU radionuclide was estimated to be greater than $0.1 \%$ of the Cs-137 activity of the waste. The remaining radionuclides apply to all LLW from the K Basins. See Appendix B, Table B-1.

${ }^{2}$ Radioactive/LLW could potentially designated as TRU or mixed waste if the sludge is incompletely removed, or if the underwater debris items are porous.

${ }^{3} \mathrm{TC}$ Metals in paint may cause this Radioactive/LLW to be designated as mixed waste.

Some debris removed from the basins may be contaminated from the sludge that has accumulated in the bottom of the basins. Because of the radionuclide contamination, PCBs, and metals concentrations in the sludge, residual sludge could potentially cause debris to designated as mixed, TRU, or mixed -TRU waste. Transuranic-designated waste is not eligible for disposal at ERDF.

Most of the accumulated sludge, therefore, will be removed through a pressure wash, conducted under water. Lead bricks and shielding, debris designated as mixed waste (e.g., painted debris exceeding TC limits), and debris that cannot be readily evaluated for compliance with LDR criteria after decontamination will be designated as hazardous, based on process knowledge, collected, and encapsulated for disposal at ERDF. Macro-encapsulation is a compliant alternative treatment technology for hazardous debris according to 40 CFR 268.45. 
Rev. 0

lon-exchange modules will be drained of free-flowing liquids and managed as debris in accordance with the ROD definition of debris. As noted above, the IXMs may be designated as hazardous for TC metals (selenium). Because the concrete shell will be considered along with the IX columns when evaluating the IXM for waste designation and radionuclides concentrations, calculations indicate that PCBs and metals concentrations with the exception of selenium are below TC levels, due to the large debris mass. The EPA agrees that the unit includes the IX column and concrete shell and constitutes a HIC which is equivalent to encapsulation (see Appendix A). The project will proceed both to confirm this interpretation and the designation of the waste.

This DQO must establish a procedure for waste designation to allow a decision as to whether the debris can be disposed at ERDF. 
HNF-6273

Rev. 0

Table 1-7. List of Preliminary Action Levels. (7 pages)

\begin{tabular}{|c|c|c|c|c|c|}
\hline \multirow[b]{2}{*}{ Weote stream } & \multirow{2}{*}{ Coc } & \multicolumn{3}{|c|}{ Prolim } & \multirow{2}{*}{ Easis: } \\
\hline & & mon & mgrikg & 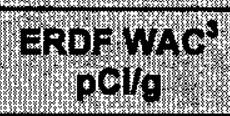 & \\
\hline Painted Debris & $\begin{array}{l}\text { radioactive COC list: } \mathrm{H}-3, \mathrm{Co}- \\
60, \mathrm{Ni}-63, \mathrm{Sr}-90, \mathrm{Sb}-125, \\
\mathrm{Cs} / \mathrm{Ba}-137, \mathrm{Pm}-147, \mathrm{Sm}-151, \\
\mathrm{Eu}-152, \mathrm{Eu} 154, \mathrm{Eu}-155, \mathrm{U}- \\
\text { 235, U-238, } \mathrm{Pu}-238, \mathrm{Pu}-239, \\
\mathrm{Pu}-240, \mathrm{Pu}-241, \mathrm{Am}-241, \mathrm{Cm}- \\
244 \\
\mathrm{TC} \text { metals -As, Ba, Cd, } \mathrm{Cr} \text {, } \\
\mathrm{Pb}, \mathrm{Hg}, \mathrm{Se}, \mathrm{Ag} \\
\text { Nonvolatile paint constituents: } \\
\text { 2-(2-methoxy)-Ethanol, 2- } \\
\text { Phthalocyanito-copper (copper } \\
\text { phthalocyanine), 2,2,4- } \\
\text { Trimethyl-1,3-- } \\
\text { pentanediolmonoisobutyrate, } \\
\text { 2-propoxyethanol, } \\
\text { Hydroxypropylmethylcellulose }{ }^{1} \\
\text { Dibutyl Phthalate } \\
\text { Naphthalene }\end{array}$ & $\begin{array}{l}\text { TC metals } \\
\text { As }-5.0, \\
\text { Ba-100.0, } \\
\text { Cd- } \\
1.0, \mathrm{Cr}- \\
5.0, \mathrm{~Pb}- \\
5.0, \mathrm{Hg}- \\
0.2, \mathrm{Se}- \\
1.0, \mathrm{Ag}- \\
5.0\end{array}$ & $\begin{array}{l}\text { organics } \\
28 \\
5.6\end{array}$ & 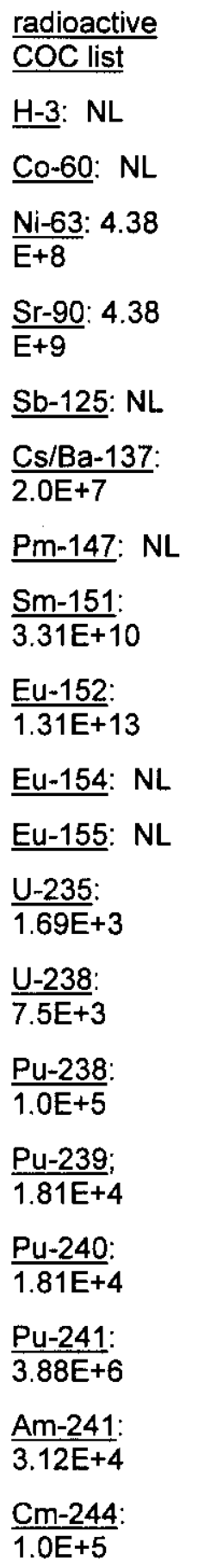 & $\begin{array}{l}40 \text { CFR } \\
261.24, \\
\text { "Toxicity } \\
\text { Characteristic" } \\
\\
40 \text { CFR } \\
268.48, \\
\text { "Universal } \\
\text { Treatment } \\
\text { Standards" } \\
\text { Washington } \\
\text { Administrative } \\
\text { Code 173-303- } \\
\text { 140 } \\
\text { Rad Limits } \\
\text { identified in } \\
\text { ERDF WAC: } \\
\text { "Environmental } \\
\text { Restoration } \\
\text { Disposal } \\
\text { Facility Waste } \\
\text { Acceptance } \\
\text { Criteria," BHI- } \\
\text { 00139, June } \\
\text { 1998. }\end{array}$ \\
\hline
\end{tabular}


Rev. 0

Table 1-7. List of Preliminary Action Levels. (7 pages)

\begin{tabular}{|c|c|c|c|c|c|}
\hline \multirow{2}{*}{ Waste Stroem } & \multirow{2}{*}{ coc } & \multicolumn{3}{|c|}{$\begin{array}{l}\text { Prolliminay } \\
\text { Action Level }\end{array}$} & \multirow{2}{*}{ Easis } \\
\hline & & moll & $\begin{array}{l}\text { Lor } \\
\text { molkg }\end{array}$ & 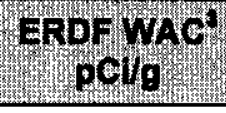 & \\
\hline $\begin{array}{c}\text { Rags } \\
\text { Contaminated } \\
\text { with Stripped } \\
\text { Paint Waste } \\
\text { (Citristrip) }\end{array}$ & $\begin{array}{l}\text { radioactive COC list }^{1} \\
\mathrm{TC} \text { metals -As, } \mathrm{Ba}, \mathrm{Cd}, \mathrm{Cr}, \mathrm{Pb} \text {, } \\
\mathrm{Hg}, \mathrm{Se}, \mathrm{Ag} \\
\text { Nonvolatile Paint Constituents: } \\
\text { 2-(2-methoxy)-Ethanol, 2- } \\
\text { Phthalocyanito-copper (copper } \\
\text { phthalocyanine), 2,2,4- } \\
\text { Trimethyl-1,3- } \\
\text { pentanediolmonoisobutyrate, } \\
\text { 2-propoxyethanol, } \\
\text { Hydroxypropylmethylcellulose }{ }^{1} \text {. } \\
\text { Citristrip: } \\
\text { Methyl-2-pyrrolidone, D- } \\
\text { Limonene }\end{array}$ & $\begin{array}{l}\text { As }-5.0, \\
\mathrm{Ba}-100.0, \\
\mathrm{Cd}- \\
1.0, \mathrm{Cr}- \\
5.0, \mathrm{~Pb}- \\
5.0, \mathrm{Hg}- \\
0.2, \mathrm{Se}- \\
1.0, \mathrm{Ag}- \\
5.0\end{array}$ & & $\begin{array}{l}\text { rad limits } \\
\text { above }\end{array}$ & $\begin{array}{l}40 \text { CFR } \\
261.24, \\
\text { "Toxicity } \\
\text { Characteristic" } \\
\text { "Environmental } \\
\text { Restoration } \\
\text { Disposal } \\
\text { Facility Waste } \\
\text { Acceptance } \\
\text { Criteria," BHI- } \\
\text { 00139, June } \\
1998 .\end{array}$ \\
\hline & $\begin{array}{l}\text { Dibutyl Phthalate } \\
\text { Naphthalene }\end{array}$ & & $\begin{array}{l}28 \\
5.6\end{array}$ & & $\begin{array}{l}40 \text { CFR } \\
268.48, \\
\text { "Universal } \\
\text { Treatment } \\
\text { Standards" } \\
\text { Washington } \\
\text { Administrative } \\
\text { Code 173-303- } \\
140\end{array}$ \\
\hline $\begin{array}{l}\text { structural } \\
\text { shielding that } \\
\text { contains haz } \\
\text { metals - lead } \\
\text { bricks, lead } \\
\text { shielding }\end{array}$ & $\mathrm{Pb}$ & $\mathrm{Pb}-5.0$ & & $\begin{array}{l}\text { rad limits } \\
\text { above }\end{array}$ & $\begin{array}{l}40 \text { CFR } \\
261.24, \\
\text { "Toxicity } \\
\text { Characteristic" } \\
\text { "Environmental } \\
\text { Restoration } \\
\text { Disposal } \\
\text { Facility Waste } \\
\text { Acceptance } \\
\text { Criteria," BHI- } \\
00139, \text { June } \\
\text { 1998. }\end{array}$ \\
\hline
\end{tabular}


HNF-6273

Rev. 0

Table 1-7. List of Preliminary Action Levels. (7 pages)

\begin{tabular}{|c|c|c|c|c|c|}
\hline \multirow{2}{*}{ waste stroam } & \multirow{2}{*}{ cos } & \multirow[b]{2}{*}{ melm } & \multicolumn{2}{|c|}{ A relmingry } & \multirow[b]{2}{*}{ Basis } \\
\hline & & & $\begin{array}{l}\text { mop } \\
\text { moge }\end{array}$ & $\begin{array}{c}\text { ERE } \\
\text { poug }\end{array}$ & \\
\hline $\begin{array}{l}\text { cartridge filters, } \\
\text { disposable } \\
\text { PPE, plastic, } \\
\text { and other trash }\end{array}$ & radioactive $\mathrm{COC}$ list & & & $\begin{array}{l}\text { rad limits } \\
\text { above }\end{array}$ & $\begin{array}{l}\text { "Environmental } \\
\text { Restoration } \\
\text { Disposal } \\
\text { Facility Waste } \\
\text { Acceptance } \\
\text { Criteria," BHI- } \\
\text { 00139, June } \\
\text { 1998. }\end{array}$ \\
\hline $\begin{array}{l}\text { materials used } \\
\text { for decon of } \\
\text { equipment: } \\
\text { cloth, paper } \\
\text { plastic }\end{array}$ & radioactive COC list & & & $\begin{array}{l}\text { rad limits } \\
\text { above }\end{array}$ & $\begin{array}{l}\text { "Environmental } \\
\text { Restoration } \\
\text { Disposal } \\
\text { Facility Waste } \\
\text { Acceptance } \\
\text { Criteria," BHI- } \\
\text { 00139, June } \\
\text { 1998. }\end{array}$ \\
\hline $\begin{array}{l}\text { process } \\
\text { equipment: } \\
\text { heat } \\
\text { exchangers, } \\
\text { piping }\end{array}$ & radioactive COC list & & & $\begin{array}{l}\text { rad limits } \\
\text { above }\end{array}$ & $\begin{array}{l}\text { "Environmental } \\
\text { Restoration } \\
\text { Disposal } \\
\text { Facility Waste } \\
\text { Acceptance } \\
\text { Criteria," BHI- } \\
\text { 00139, June } \\
\text { 1998. }\end{array}$ \\
\hline $\begin{array}{c}\text { Unpainted } \\
\text { demolition } \\
\text { debris, } \\
\text { structural steel, } \\
\text { rocks, gravel, } \\
\text { metal, glass, } \\
\text { concrete, } \\
\text { ceramic, bricks, } \\
\text { roofing } \\
\text { material, wood } \\
\text { drywall, siding }\end{array}$ & radioactive $\mathrm{COC}$ list & & & $\begin{array}{l}\text { rad limits } \\
\text { above }\end{array}$ & $\begin{array}{l}\text { "Environmental } \\
\text { Restoration } \\
\text { Disposal } \\
\text { Facility Waste } \\
\text { Acceptance } \\
\text { Criteria," BHI- } \\
\text { 00139, June } \\
\text { 1998. }\end{array}$ \\
\hline $\begin{array}{c}\text { materials } \\
\text { collected } \\
\text { during general } \\
\text { housekeeping: } \\
\text { soil, sawdust, } \\
\text { vegetation, } \\
\text { debris, glass, } \\
\text { plastic }\end{array}$ & radioactive $\mathrm{COC}$ list & & & $\begin{array}{l}\text { rad limits } \\
\text { above }\end{array}$ & $\begin{array}{l}\text { "Environmental } \\
\text { Restoration } \\
\text { Disposal } \\
\text { Facility Waste } \\
\text { Acceptance } \\
\text { Criteria," BHI- } \\
\text { 00139, June } \\
\text { 1998. }\end{array}$ \\
\hline
\end{tabular}


Rev. 0

Table 1-7. List of Preliminary Action Levels. (7 pages)

\begin{tabular}{|c|c|c|c|c|c|}
\hline \multirow{2}{*}{$w=s t e$ stregm } & \multirow{2}{*}{ cog } & \multicolumn{3}{|c|}{ Action } & \multirow{2}{*}{ Basts } \\
\hline & & Tom & $\begin{array}{l}\text { LDR } \\
\text { mgikg }\end{array}$ & 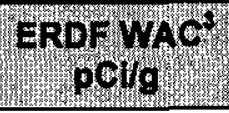 & \\
\hline HEPA filters & radioactive $\mathrm{COC}$ list & & & $\begin{array}{l}\text { rad limits } \\
\text { above }\end{array}$ & $\begin{array}{l}\text { "Environmental } \\
\text { Restoration } \\
\text { Disposal } \\
\text { Facility Waste } \\
\text { Acceptance } \\
\text { Criteria," BHI- } \\
\text { 00139, June } \\
\text { 1998. }\end{array}$ \\
\hline $\begin{array}{l}\text { structural steel } \\
\text { - fuel storage } \\
\text { racks \& } \\
\text { bulkheads, } \\
\text { structures used } \\
\text { for fuel } \\
\text { handling }\end{array}$ & $\begin{array}{l}\text { radioactive COC list } \\
\mathrm{PCBs}, \mathrm{TC} \text { metals }-\mathrm{As}, \mathrm{Ba}, \mathrm{Cd}_{1} \\
\mathrm{Cr}, \mathrm{Pb}, \mathrm{Hg}, \mathrm{Se}, \mathrm{Ag}\end{array}$ & $\begin{array}{l}\mathrm{As}-5.0 \\
\mathrm{Ba}-100.0, \\
\mathrm{Cd}- \\
1.0, \mathrm{Cr}- \\
5.0, \mathrm{~Pb}- \\
5.0, \mathrm{Hg}- \\
0.2, \mathrm{Se}- \\
1.0, \mathrm{Ag}- \\
5.0\end{array}$ & & $\begin{array}{l}\text { rad limits } \\
\text { above } \\
\text { PCBs - } \\
500 \mathrm{mg} / \mathrm{kg}\end{array}$ & $\begin{array}{l}40 \text { CFR } \\
261.24, \\
\text { "Toxicity } \\
\text { Characteristic" } \\
\text { "Environmental } \\
\text { Restoration } \\
\text { Disposal } \\
\text { Facility Waste } \\
\text { Acceptance } \\
\text { Criteria," BHI- } \\
\text { 00139, June } \\
1998 .\end{array}$ \\
\hline $\begin{array}{l}\text { process } \\
\text { equipment - } \\
\text { pumps, old } \\
\text { canister } \\
\text { washer, piping } \\
\text { and piping } \\
\text { components, } \\
\text { rubber hoses }\end{array}$ & $\begin{array}{l}\text { radioactive COC list } \\
\mathrm{PCBs}, \mathrm{TC} \text { metals - } \mathrm{As}, \mathrm{Ba}, \mathrm{Cd} \\
\mathrm{Cr}, \mathrm{Pb}, \mathrm{Hg}, \mathrm{Se}, \mathrm{Ag}\end{array}$ & $\begin{array}{l}\text { As }-5.0, \\
\mathrm{Ba}-100.0, \\
\mathrm{Cd}- \\
1.0, \mathrm{Cr}- \\
5.0, \mathrm{~Pb}- \\
5.0, \mathrm{Hg}- \\
0.2, \mathrm{Se}- \\
1.0, \mathrm{Ag}- \\
5.0\end{array}$ & & 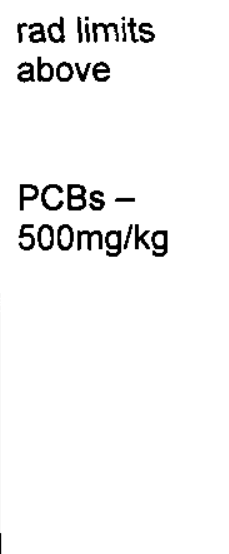 & $\begin{array}{l}40 \text { CFR } \\
261.24, \\
\text { "Toxicity } \\
\text { Characteristic" } \\
\\
\text { "Environmental } \\
\text { Restoration } \\
\text { Disposal } \\
\text { Facility Waste } \\
\text { Acceptance } \\
\text { Criteria," BHI- } \\
\text { 00139, June } \\
\text { 1998. }\end{array}$ \\
\hline
\end{tabular}


Rev. 0

Table 1-7. List of Preliminary Action Levels. (7 pages)

\begin{tabular}{|c|c|c|c|c|c|}
\hline \multirow[b]{2}{*}{ Whete stroam } & \multirow{2}{*}{ coc } & \multicolumn{3}{|c|}{ Action Level } & \multirow{2}{*}{ B $\mathrm{sic}$} \\
\hline & & $\begin{array}{l}\text { TC } \\
\text { ngn }\end{array}$ & mor & $\begin{array}{l}\text { ERP } \\
\text { porg }\end{array}$ & \\
\hline $\begin{array}{c}\text { miscellaneous } \\
\text { debris - } \\
\text { electrical } \\
\text { cables, light } \\
\text { fixtures, long } \\
\text { tools, brushes, } \\
\text { PPE, metal, } \\
\text { plastic }\end{array}$ & $\begin{array}{l}\text { radioactive } \mathrm{COC} \text { list } \\
\mathrm{PCBs}, \mathrm{TC} \text { metals }-\mathrm{As}, \mathrm{Ba}, \mathrm{Cd} \text {, } \\
\mathrm{Cr}, \mathrm{Pb}, \mathrm{Hg}, \mathrm{Se}, \mathrm{Ag}\end{array}$ & $\begin{array}{l}\text { As }-5.0 \text {, } \\
\mathrm{Ba}-100.0, \\
\mathrm{Cd}- \\
1.0, \mathrm{Cr}- \\
5.0, \mathrm{~Pb}- \\
5.0, \mathrm{Hg}- \\
0.2, \mathrm{Se}- \\
1.0, \mathrm{Ag}- \\
5.0\end{array}$ & & 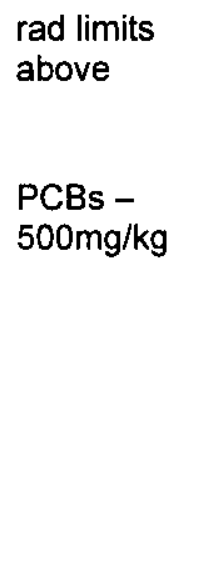 & $\begin{array}{l}40 \text { CFR } \\
261.24, \\
\text { "Toxicity } \\
\text { Characteristic" } \\
\\
\text { "Environmental } \\
\text { Restoration } \\
\text { Disposal } \\
\text { Facility Waste } \\
\text { Acceptance } \\
\text { Criteria," BHI- } \\
\text { 00139, June } \\
\text { 1998. }\end{array}$ \\
\hline $\begin{array}{c}\text { Canisters/canis } \\
\text { ter lids }\end{array}$ & $\begin{array}{l}\text { radioactive COC list } \\
\mathrm{PCBs}, \mathrm{TC} \text { metals }-\mathrm{As}, \mathrm{Ba}, \mathrm{Cd} \text {, } \\
\mathrm{Cr}, \mathrm{Pb}, \mathrm{Hg}, \mathrm{Se}, \mathrm{Ag}\end{array}$ & $\begin{array}{l}\text { As }-5.0 \\
\mathrm{Ba}-100.0 \\
\mathrm{Cd}- \\
1.0, \mathrm{Cr}- \\
5.0, \mathrm{~Pb}- \\
5.0, \mathrm{Hg}- \\
0.2, \mathrm{Se}- \\
1.0, \mathrm{Ag}- \\
5.0\end{array}$ & & $\begin{array}{l}\begin{array}{l}\text { rad limits } \\
\text { above }\end{array} \\
\text { PCBs - } \\
500 \mathrm{mg} / \mathrm{kg}\end{array}$ & $\begin{array}{l}40 \text { CFR } \\
261.24, \\
\text { "Toxicity } \\
\text { Characteristic" } \\
\text { "Environmental } \\
\text { Restoration } \\
\text { Disposal } \\
\text { Facility Waste } \\
\text { Acceptance } \\
\text { Criteria," BHI- } \\
\text { 00139, June } \\
\text { 1998. }\end{array}$ \\
\hline IXMs & $\begin{array}{l}\text { radioactive COC list } \\
\mathrm{PCBs}, \mathrm{TC} \text { metals - As, } \mathrm{Ba}, \mathrm{Cd} \text {, } \\
\mathrm{Cr}, \mathrm{Pb}, \mathrm{Hg}, \mathrm{Se}, \mathrm{Ag}\end{array}$ & $\begin{array}{l}\text { As }-5.0, \\
\text { Ba-100.0, } \\
\text { Cd- } \\
1.0, \mathrm{Cr}- \\
5.0, \mathrm{~Pb}- \\
5.0, \mathrm{Hg}- \\
0.2, \mathrm{Se}- \\
1.0, \mathrm{Ag}- \\
5.0\end{array}$ & & $\begin{array}{l}\text { rad limits } \\
\text { above }\end{array}$ & $\begin{array}{l}40 \text { CFR } \\
261.24, \\
\text { "Toxicity } \\
\text { Characteristic" } \\
\\
\text { "Environmental } \\
\text { Restoration } \\
\text { Disposal } \\
\text { Facility Waste } \\
\text { Acceptance } \\
\text { Criteria," BHI- } \\
\text { 00139, June } \\
\text { 1998. }\end{array}$ \\
\hline
\end{tabular}


HNF-6273

Rev. 0

Table 1-7. List of Preliminary Action Levels. (7 pages)

\begin{tabular}{|c|c|c|c|c|c|}
\hline \multirow[b]{2}{*}{ Whatestream } & \multirow{2}{*}{ cog } & \multicolumn{3}{|c|}{ Actonlingry } & \multirow{2}{*}{ Easts } \\
\hline & & $\begin{array}{l}\mathrm{TC} \\
\mathrm{mgl}\end{array}$ & Mor & $\begin{array}{c}\text { ERPF } \\
\text { parg }\end{array}$ & \\
\hline $\begin{array}{c}\text { floor } \\
\text { tiles/ceiling } \\
\text { tiles; sprayed } \\
\text { on ceiling } \\
\text { texture or } \\
\text { acoustic } \\
\text { surface } \\
\text { coatings }\end{array}$ & $\begin{array}{l}\mathrm{Pb} \text { if painted } \\
\text { asbestos }^{2} \\
\text { radioactive COC list }\end{array}$ & $\mathrm{Pb}-5.0$ & & $\begin{array}{l}\text { rad limits } \\
\text { above }\end{array}$ & $\begin{array}{l}40 \text { CFR } \\
261.24, \\
\text { "Toxicity } \\
\text { Characteristic" } \\
\\
\text { "Environmental } \\
\text { Restoration } \\
\text { Disposal } \\
\text { Facility Waste } \\
\text { Acceptance } \\
\text { Criteria," BHI- } \\
\text { 00139, June } \\
\text { 1998. }\end{array}$ \\
\hline $\begin{array}{l}\text { pipe and duct } \\
\text { insulation and } \\
\text { insulation } \\
\text { mastic; mastic } \\
\text { used as } \\
\text { adhesive for } \\
\text { plastic } \\
\text { baseboard } \\
\text { moldings }\end{array}$ & $\begin{array}{l}\text { asbestos }^{2} \\
\text { radioactive COC list }\end{array}$ & & & $\begin{array}{l}\text { rad limits } \\
\text { above }\end{array}$ & $\begin{array}{l}\text { "Environmental } \\
\text { Restoration } \\
\text { Disposal } \\
\text { Facility Waste } \\
\text { Acceptance } \\
\text { Criteria," BHI- } \\
\text { 00139, June } \\
\text { 1998. }\end{array}$ \\
\hline $\begin{array}{l}\text { mineral based } \\
\text { building } \\
\text { insulation in } \\
\text { walls and } \\
\text { ceilings }\end{array}$ & $\begin{array}{l}\text { asbestos }^{2} \\
\text { radioactive COC list }\end{array}$ & & & $\begin{array}{l}\text { rad limits } \\
\text { above }\end{array}$ & $\begin{array}{l}\text { "Environmental } \\
\text { Restoration } \\
\text { Disposal } \\
\text { Facility Waste } \\
\text { Acceptance } \\
\text { Criteria," BHI- } \\
\text { 00139, June } \\
1998 . \\
\end{array}$ \\
\hline $\begin{array}{c}\text { asbestos board } \\
\text { (transite) used } \\
\text { in walls, } \\
\text { ceilings, siding }\end{array}$ & $\begin{array}{l}\text { asbestos }^{2} \\
\text { radioactive COC list }\end{array}$ & & & $\begin{array}{l}\text { rad limits } \\
\text { above }\end{array}$ & $\begin{array}{l}\text { "Environmental } \\
\text { Restoration } \\
\text { Disposal } \\
\text { Facility Waste } \\
\text { Acceptance } \\
\text { Criteria," BHI- } \\
\text { O0139, June } \\
1998 .\end{array}$ \\
\hline
\end{tabular}


HNF-6273

Rev. 0

Table 1-7. List of Preliminary Action Levels. (7 pages)

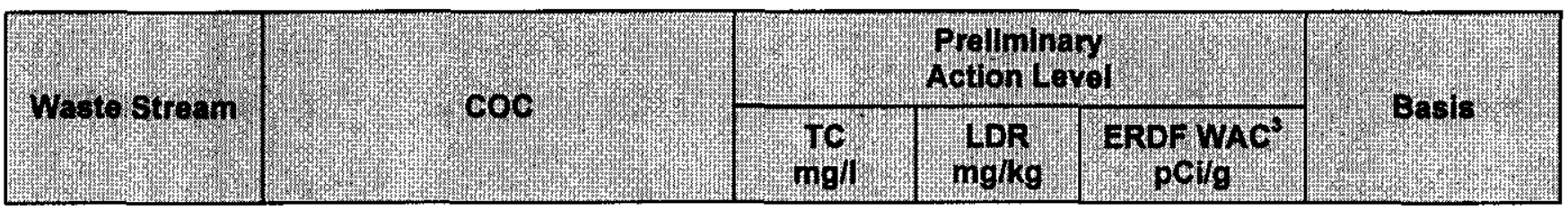

${ }^{1}$ Nonvolatile paint constituent action levels will be based on a calculation for toxicity, as provided in Washington Administrative Code 173-303-100 (5).

${ }^{2}$ No action limits have been specified for asbestos. Note that ACMs shall be managed in accordance with 40 CFR 61.140 through 157. Detailed information on packaging and shipping can be found in ERDF Supplemental WAC (BHI 1997).

${ }^{3}$ The ERDF WAC (BHI 1998) radionuclide concentration limits are listed in units of $\mathrm{Ci} / \mathrm{m}^{3}$. These units have been converted to $\mathrm{pCi} / \mathrm{g}$ using the following conversion factors:

$\mathrm{Ci}=10^{12} \mathrm{pCi}$

$1 \mathrm{~m} 3=10^{6} \mathrm{cc}$

$1.6 \mathrm{~g} / \mathrm{cc}=$ Approximate estimated package density of waste. Actual waste densities will vary and final calculation will use actual weights and volumes.

the resulting unit is $\mathrm{pCi} / \mathrm{g}$.

${ }^{4}$ The listed WAC are the lowest limits for the COCs from consideration of Table 3 of the ERDF WAC, Class C limits from 10 CFR 61 and the TRU limit of $100 \mathrm{nCi} / \mathrm{g}$. 


\subsection{STEP 2 - IDENTIFY THE DECISION}

The purpose of DQO Step 2 is to define the principal study questions (PSQs) that need to be resolved to address the problem(s) identified in DQO Step 1 and to define the alternative actions that would result from the resolution of the PSQs. The PSQs and alternative actions are then combined into decision statements (DSs) that provide a basis for gathering information.

\section{$2.1 \quad$ PRINCIPAL STUDY QUESTIONS}

The PSQs are basic DQO questions that will require measurements (e.g., physical, chemical, or radiological data) to resolve. An initial operating assumption for this project is that all debris is radiologically contaminated. Therefore, PSQs are directed towards a determination of the level of contamination and whether debris is mixed waste, i.e., also contaminated with hazardous/dangerous waste constituents.

\subsection{ALTERNATIVE ACTIONS}

Table 2-2 identifies the alternative actions that could be taken once the PSQs have been resolved.

Table 2-1. Principal Study Questions.

\begin{tabular}{|c|l|}
\hline PSO & \multicolumn{1}{c}{ Princlpal Study ouestion } \\
\hline 1 & $\begin{array}{l}\text { Is the material radiologically contaminated with constituents that cause it to be regulated } \\
\text { as TRU? }\end{array}$ \\
\hline 2 & Does the material's radiological activity exceed the ERDF WAC (BHI 1998) limits? \\
\hline 3 & Is the material a dangerous waste ${ }^{\mathrm{a}, \mathrm{b}}$, PCB-regulated, or asbestos waste? \\
\hline $3 a$ & Is the material a TC or Washington toxic waste? \\
\hline $3 b$ & Does the material contain asbestos? \\
\hline 4 & Is the material land disposal restricted? \\
\hline
\end{tabular}

a The definition of dangerous waste also includes hazardous waste.

${ }^{b}$ Process knowledge excludes waste being reactive, ignitable, or corrosive (see, for example, DOE-RL 1999b). 
Rev. 0

Table 2-2. Alternative Actions.

\begin{tabular}{|c|c|c|}
\hline Psow & 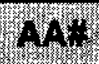 & Altemat vo Action \\
\hline 1 & 1 & $\begin{array}{l}\text { The material is not radiologically contaminated above TRU waste levels and will } \\
\text { be considered for disposition at the ERDF. }\end{array}$ \\
\hline 1 & 2 & $\begin{array}{l}\text { The material is radiologically contaminated above TRU waste levels and will not } \\
\text { be disposed at ERDF. Waste will be segregated for alternate disposal. }\end{array}$ \\
\hline 2 & 1 & $\begin{array}{l}\text { The radiological activity of the material does not exceed the ERDF WAC } \\
\text { (BHI 1998) limits. It will be evaluated per discussions \#3 and } 4 \text {. }\end{array}$ \\
\hline 2 & 2 & $\begin{array}{l}\text { The radiological activity of the material exceeds the ERDF WAC (BHI 1998) } \\
\text { limits. It will be evaluated by ERDF on a case by case basis. If the waste cannot } \\
\text { be accepted at ERDF, alternative disposal locations will be identified. It is likely } \\
\text { that the alternative would be packaging and shipment to the CWC. The material } \\
\text { will also be evaluated per discussions } \# 3 \text { and } 4 \text { below if it is determined } \\
\text { appropriate to go to ERDF. }\end{array}$ \\
\hline $3 a$ & 1 & $\begin{array}{l}\text { The material is a TC waste and receives a characteristic waste code. The waste } \\
\text { will be treated through macro-encapsulation before disposal. }\end{array}$ \\
\hline $3 a$ & 2 & The material is not a TC waste and is disposed at ERDF without treatment. \\
\hline $3 a$ & 3 & $\begin{array}{l}\text { If the material exceeds state dangerous toxic criteria using calculations in } \\
\text { Washington Administrative Code } 173-303-100(5) \text {, the waste will be disposed in } \\
\text { ERDF. (NOTE: RCW } 70.105 .050 \text { authorizes the disposal of extremely } \\
\text { hazardous waste (EHW) that contains radioactive components after appropriate } \\
\text { treatment at DOE facilities.) }\end{array}$ \\
\hline $3 a$ & 4 & $\begin{array}{l}\text { If the material is below the state dangerous toxic criteria using calculations in } \\
\text { Washington Administrative Code } 173-303-100(5) \text {, the waste will be disposed in } \\
\text { ERDF. }\end{array}$ \\
\hline $3 b$ & 1 & $\begin{array}{l}\text { The material is regulated due to asbestos content and will be managed } \\
\text { appropriately prior to disposal. }\end{array}$ \\
\hline $3 b$ & 2 & The material is not regulated due to asbestos content. \\
\hline 4 & 1 & $\begin{array}{l}\text { The material is land disposal restricted. Treatment in the form of macro- } \\
\text { encapsulation is imposed on the material prior to disposal. }\end{array}$ \\
\hline 4 & 2 & $\begin{array}{l}\text { The material is not land disposal restricted. Treatment is not required for the } \\
\text { material prior to disposal }\end{array}$ \\
\hline
\end{tabular}

\subsection{DECISION STATEMENTS}

Table 2-3 uses the PSQs and alternative actions to create decision statements (DSs) using the following format: Determine whether or not [unknown conditions/issues/criteria from the PSQ] require (or support) [taking alternative actions]. 
HNF-6273

Rev. 0

Table 2-3. Decision Statements.

\begin{tabular}{|c|l|}
\hline DSH & \multicolumn{1}{|c|}{ Docision Statement } \\
\hline 1 & $\begin{array}{l}\text { Determine whether the debris exceeds the radiological contamination limits for TRU waste } \\
\text { material. }\end{array}$ \\
\hline 2 & Determine whether the radionuclide content of the debris exceeds the WAC for the ERDF. \\
\hline 3 & Determine whether the material designates as dangerous, TC, or is asbestos waste. \\
\hline $3 a$ & $\begin{array}{l}\text { Determine whether the characteristic waste code for toxicity or state dangerous waste toxic } \\
\text { applies to the material. }\end{array}$ \\
\hline $3 b$ & Determine whether the material is regulated due to asbestos content. \\
\hline 4 & Determine whether LDRs require treatment prior to disposal. \\
\hline
\end{tabular}


Figure 2-1. Generic Waste Disposition Decision Logic.

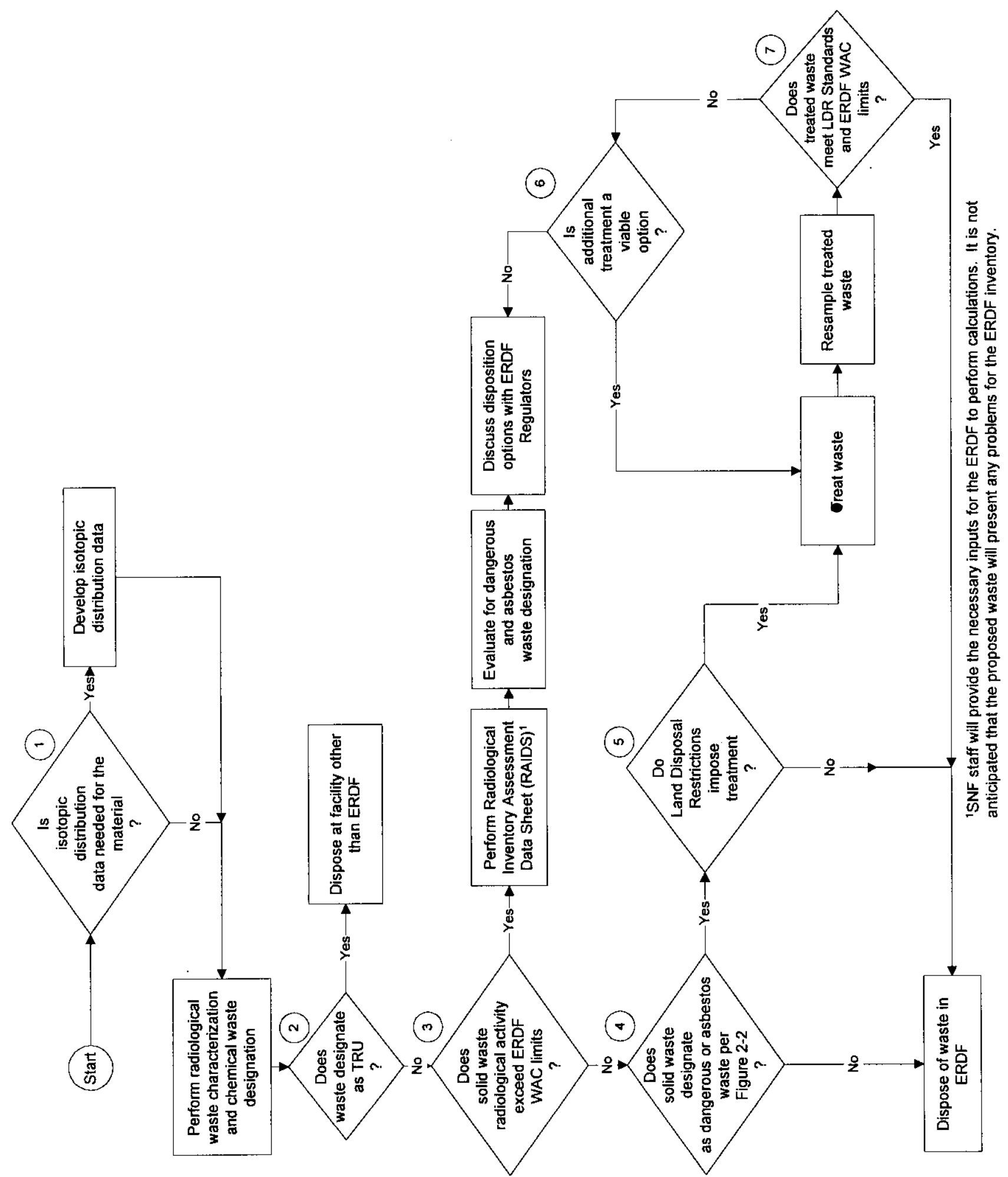


HNF-6273

Rev. 0

Figure 2-2. Chemical Waste Designation Decision Logic.

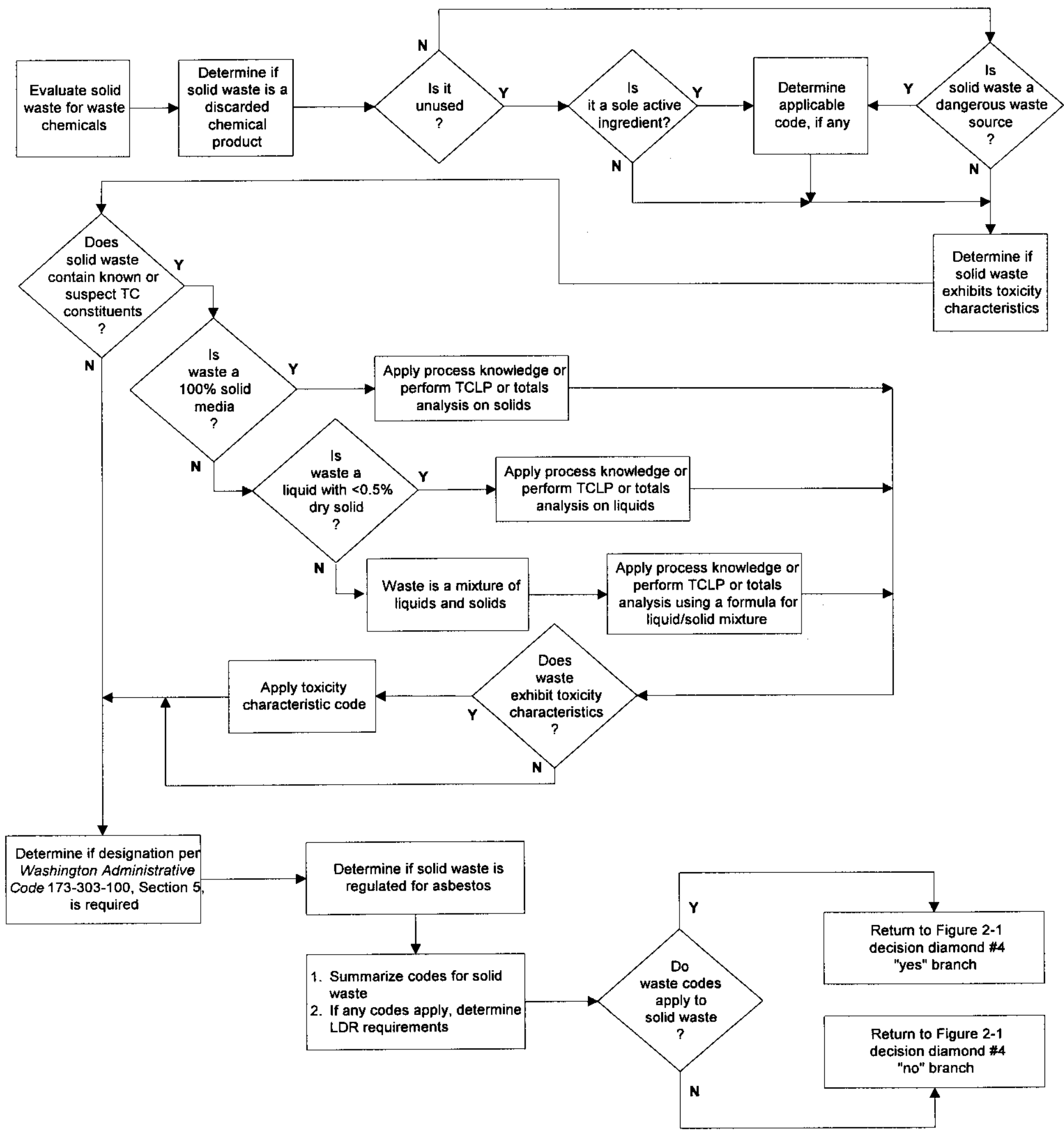


HNF-6273

Rev. 0 
Rev. 0

\subsection{STEP 3 - IDENTIFY INPUTS TO THE DECISION}

The purpose of DQO Step 3 is to identify the type of data needed to resolve each of the DSs identified in Step 2, as well as the analytical performance requirements (e.g., practical quantitation limit [PQL] requirement, precision, and accuracy) for the data. If it is determined that the required data do not already exist, the data may be obtained by field measurements.

\subsection{REQUIRED INFORMATION}

Tables 3-1a through 3-3 deal with the information required to resolve the DSs. Existing data are evaluated for use, and computational and field measurement methods are identified that can supply the needed data.

Table 3-1 lists the data required for each DS. Source references for the data are provided along with a qualitative assessment as to whether or not the data are of sufficient quality to resolve the corresponding DS.

Table 3-1. Required Information and Reference Sources.

\begin{tabular}{|c|c|c|c|c|c|}
\hline Dst & Required bata & $\begin{array}{l}\text { Doos } \\
\text { Data } \\
\text { Exint? }\end{array}$ & sourne Rorerenco & surforent & $\begin{array}{l}\text { Tore } \\
\text { Info } \\
\text { Rog d? } \\
(\mathrm{YNI})\end{array}$ \\
\hline 1 & $\begin{array}{l}\text { Estimation of the TRU } \\
\text { content }(\mathrm{nCi} / \mathrm{g}) \text { of each } \\
\text { waste package. }\end{array}$ & $\mathrm{Y}$ & See Section 7.0 & $\mathrm{Y}$ & $\mathrm{Y}$ \\
\hline 2 & $\begin{array}{l}\text { Estimation of the } \\
\text { radionuclide }\left(\mathrm{Ci} / \mathrm{m}^{3}\right) \\
\text { content of each waste } \\
\text { package. }\end{array}$ & $\bar{Y}$ & See Section 7.0. & $\bar{Y}$ & $\bar{Y}$ \\
\hline $\begin{array}{l}3 a \\
3 b\end{array}$ & \multicolumn{5}{|l|}{ See Table 3-1a } \\
\hline 4 & $\begin{array}{l}\text { Determine whether } \\
\text { LDRs require debris to } \\
\text { be treated prior to } \\
\text { disposal. }\end{array}$ & $\bar{Y}$ & $\begin{array}{l}\text { NHC-96-101,"Analytical Report } \\
\text { for K Basin Paint -FT6112," } \\
\text { September 11, 1996. } \\
\text { Analytical Report for FAST } \\
\text { Project FD1-7021, "K Basin } \\
\text { Crane Removal," July 15, } 1997 . \\
\text { Discussion of total metals } \\
\text { concentrations in K-Basin } \\
\text { Sludge: KBSN-96-01, "K Basin } \\
\text { Sludge Waste Stream Profile } \\
\text { Sheet," 6/26/96. } \\
\text { Toxic metals and PCBs potential } \\
\text { to sorb onto the IX column, } \\
\text { calculations in Appendix C. }\end{array}$ & $\bar{Y}$ & $\bar{Y}$ \\
\hline
\end{tabular}


HNF-6273

Rev. 0

\begin{tabular}{|c|c|c|c|c|c|c|c|}
\hline $\begin{array}{l}64 \\
689 \\
6\end{array}$ & & $>$ & & $\mathbf{z}$ & $\mathbf{z}$ & 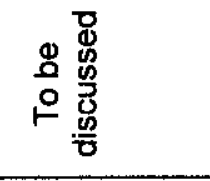 & $>$ \\
\hline$\frac{5}{5}=2$ & & $>$ & & $>$ & $\nu$ & 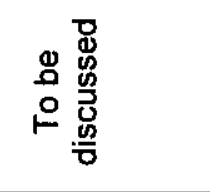 & $z$ \\
\hline 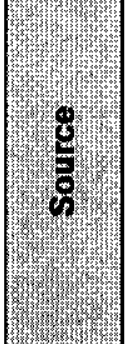 & & 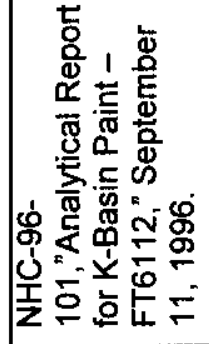 & 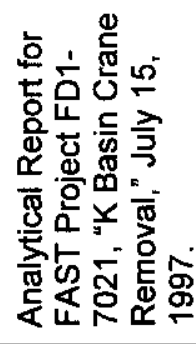 & 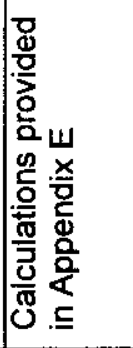 & 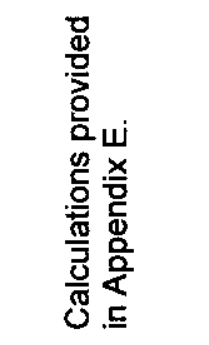 & 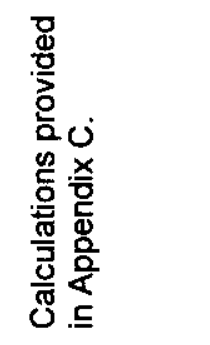 & $\frac{\aleph}{\mathbf{z}}$ \\
\hline$x^{2}{ }^{2}$ & & $>$ & & $>$ & $\succ$ & $>$ & $z$ \\
\hline 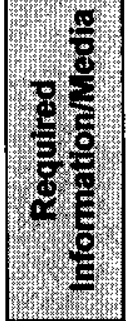 & & 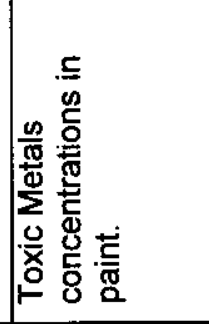 & & 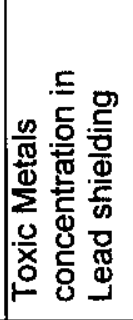 & 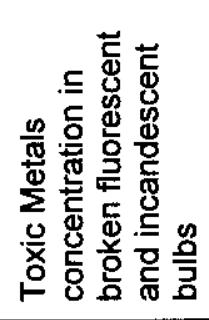 & 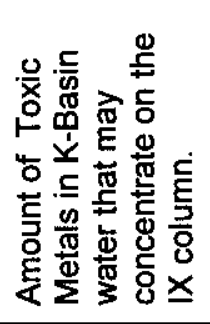 & 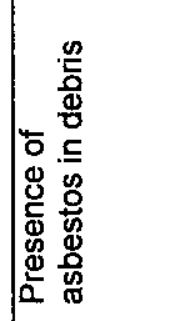 \\
\hline$x^{2}$ & & 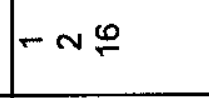 & & $m$ & $\nabla$ & 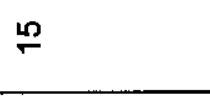 & $\dddot{2} \div \stackrel{\infty}{\div}$ \\
\hline 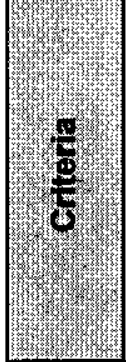 & 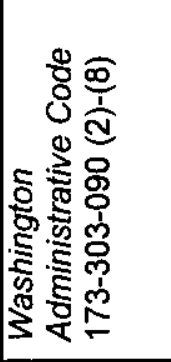 & 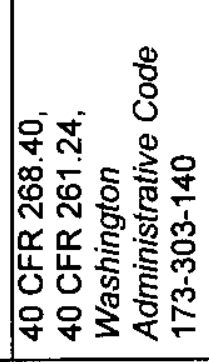 & & & & & 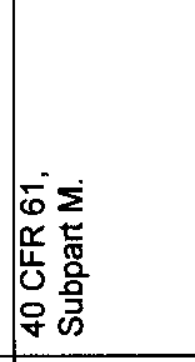 \\
\hline 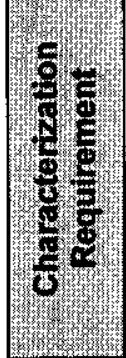 & 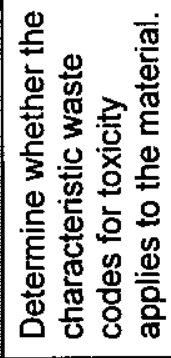 & & & & & & 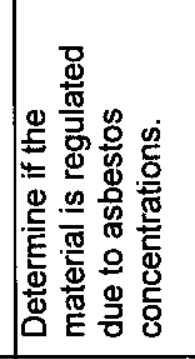 \\
\hline 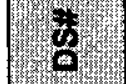 & ల్ల & & & & & & ले \\
\hline
\end{tabular}


Table 3-2 identifies the DSs where existing data either do not exist or are of insufficient quality to resolve the DSs. For those cases, Table 3-2 identifies possible computational and/or surveying/sampling methods that could be used to obtain the required data.

Table 3-2. Information Required to Resolve the Decision Statements. (2 pages)

\begin{tabular}{|c|c|c|c|c|}
\hline Pon & Infomaronal Noed & Required Bata & Computational & $\begin{array}{l}\text { Avollabio } \\
\text { Suryaysampling } \\
\text { Mothods }\end{array}$ \\
\hline 1 & $\begin{array}{l}\text { Estimate of the } \\
\text { upper bound of the } \\
\text { TRU content of the } \\
\text { waste package, } \\
\text { compare to TRU } \\
\text { criteria. }\end{array}$ & $\begin{array}{l}\text { TRU isotopic data. } \\
\mathrm{nCi} / \mathrm{g}\end{array}$ & $\begin{array}{l}\text { Apply dose-to- } \\
\text { curie conversion } \\
\text { to obtain Cs-137 } \\
\text { levels in the } \\
\text { waste. Apply } \\
\text { predetermined } \\
\text { radionuclide } \\
\text { ratios to } \\
\text { estimate the } \\
\text { amount of each } \\
\text { of the COCs } \\
\text { from the Cs- } \\
137 .\end{array}$ & \multirow[t]{2}{*}{$\begin{array}{l}\text { Dose meter, gamma } \\
\text { survey, or NDA of } \\
\text { individual pieces of } \\
\text { debris or appropriate } \\
\text { containers of debris. } \\
\text { Media sampling and } \\
\text { laboratory analysis of } \\
\text { debris. }\end{array}$} \\
\hline 2 & $\begin{array}{l}\text { Estimate of the } \\
\text { radionuclide content } \\
\text { of the waste } \\
\text { package. Compare } \\
\text { to ERDF WAC } \\
\text { (BHI 1998). }\end{array}$ & $\begin{array}{l}\text { Radiological survey data } \\
\text { for direct counting, } \\
\text { analytical results of } \\
\text { samples. } \\
\mathrm{Ci} / \mathrm{m}^{3} \text { or } \mathrm{pCi} / \mathrm{g}\end{array}$ & $\begin{array}{l}\text { Apply dose-to- } \\
\text { curie conversion } \\
\text { to obtain Cs-137 } \\
\text { levels in the } \\
\text { waste. Apply } \\
\text { predetermined } \\
\text { radionuclide } \\
\text { ratios to } \\
\text { estimate the } \\
\text { amount of each } \\
\text { of the COCs } \\
\text { from the Cs- } \\
137 .\end{array}$ & \\
\hline $3 a$ & $\begin{array}{l}\text { Toxic Metals } \\
\text { concentrations in } \\
\text { Lead shielding } \\
\text { Toxic Metals } \\
\text { concentration in } \\
\text { broken fluorescent } \\
\text { and incandescent } \\
\text { bulbs }\end{array}$ & Metals concentrations & $\begin{array}{l}\text { See Appendix } \\
\text { D. }\end{array}$ & Process knowledge \\
\hline $3 b$ & $\begin{array}{l}\text { Presence of } \\
\text { asbestos in debris. }\end{array}$ & $\begin{array}{l}\text { Presence of asbestos } \\
\text { fiber } \\
\text { Process knowledge }\end{array}$ & N/A & $\begin{array}{l}\text { Visual inspection of } \\
\text { building materials by a } \\
\text { certified AHERA } \\
\text { inspector. } \\
\text { Process knowledge }\end{array}$ \\
\hline
\end{tabular}


HNF-6273

Rev. 0

Table 3-2. Information Required to Resolve the

Decision Statements. (2 pages)

\begin{tabular}{|c|c|c|c|c|}
\hline Bst & Informational Need & Requirod Data & computatongl & $\begin{array}{l}\text { Ayciablo } \\
\text { Sungysampling } \\
\text { Mothods }\end{array}$ \\
\hline 4 & $\begin{array}{l}\text { (1)Determine if toxic } \\
\text { metals } \\
\text { concentrations in } \\
\text { sludge exceed TC } \\
\text { limits and amount of } \\
\text { sludge present on } \\
\text { debris. (2) } \\
\text { Determine if toxic } \\
\text { metals in painted } \\
\text { debris, stripped } \\
\text { paint, lead shielding, } \\
\text { and broken } \\
\text { fluorescent and } \\
\text { incandescent bulbs } \\
\text { exceed TC limits. } \\
\text { (3) Determine if } \\
\text { IXMs contain } \\
\text { concentrated toxic } \\
\text { metals at levels } \\
\text { above TC limits. }\end{array}$ & $\begin{array}{l}\text { Concentrations of toxic } \\
\text { metals and PCBs }\end{array}$ & N/A & $\begin{array}{l}\text { Items will be visually } \\
\text { inspected. Painted } \\
\text { debris, stripped paint, } \\
\text { lead shielding, broken } \\
\text { fluorescent and } \\
\text { incandescent bulbs } \\
\text { will be segregated, } \\
\text { collected and macro- } \\
\text { encapsulated prior to } \\
\text { land disposal. } \\
\text { Process knowledge } \\
\text { Historical data }\end{array}$ \\
\hline
\end{tabular}

Table 3-3 identifies computational methods that may used to provide the data needed to resolve the DSs.

\subsection{FIELD MEASURMENT METHODS AND ANALYTICAL PERFORMANCE REQUIREMENTS}

The field measurement methods and analytical performance requirements that will support resolution of the DSs are developed in Tables 3-4 and 3-5. For Table 3-4, refer to Table 1-7 for a summary of the preliminary action levels for each of the COCs for each individual WS. Confirm that appropriate measurement methods exist to provide the necessary data. The possible limitations associated with each of these methods are also provided along with the estimated cost. 
HNF-6273

Rev. 0

Table 3-3. List of Potential Computational Methods.

\begin{tabular}{|c|c|c|c|}
\hline pst & Computatonal & sourcoluthor & Appliction to swory \\
\hline 1 & $\begin{array}{l}\text { Conversion from dose } \\
\text { rate or NDA gamma } \\
\text { and neutron results to } \\
\text { estimated TRU } \\
\text { radionuclide levels in } \\
\text { the waste package. }\end{array}$ & $\begin{array}{l}\text { TBD, Depends on the } \\
\text { vendor that performs } \\
\text { the NDA. Past data, } \\
\text { see PNNL (1999) and } \\
\text { WHC (1996a, 1996b). }\end{array}$ & \\
\hline 2 & $\begin{array}{l}\text { Conversion from dose } \\
\text { rate or gamma } \\
\text { surveys to an estimate } \\
\text { of the amount of COC } \\
\text { that is in each waste } \\
\text { package. }\end{array}$ & $\begin{array}{l}\text { WHC (1996b), WHC } \\
\text { (1996a) }\end{array}$ & $\begin{array}{l}\text { Direct sampling and measurement of all } \\
\text { COC radionuclides on the waste in each } \\
\text { waste package is not feasible. } \\
\text { Concentrations }\left(\mathrm{Ci} / \mathrm{m}^{3}\right) \text { of } \mathrm{COC} \text { in the } \\
\text { waste package will be inferred from } \\
\text { external dose or gamma surveys of the } \\
\text { packaged waste. Confirmation samples } \\
\text { or additional external NDA measurements } \\
\text { may be obtained and analyzed if } \\
\text { anomalies (e.g. high dose rate, isotopic } \\
\text { ratio changes) are observed. }\end{array}$ \\
\hline $3 a$ & & $\begin{array}{l}\text { Calculations in } \\
\text { Appendix } C, D \text {, and } E .\end{array}$ & $\begin{array}{l}\text { Appendix C calculates the potential of the } \\
\text { IX column to sorb PCBs and toxic metals } \\
\text { from K-Basin water analyses. } \\
\text { Appendices D and E calculate the } \\
\text { potential lead content of debris and } \\
\text { concentrations of TC metals and PCBs in } \\
\text { sludge, respectively. Results are } \\
\text { compared with TC levels for metals and } \\
\text { PCBs. }\end{array}$ \\
\hline
\end{tabular}


HNF-6273

Rev. 0

Table 3-4. Potentially Appropriate Measurement Methods.

\begin{tabular}{|c|c|c|c|c|c|}
\hline Ds: & 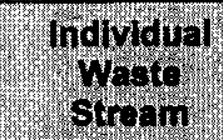 & (nomatonal & Polentialy Approprate & $\begin{array}{l}\text { Possible } \\
\text { Limitions or } \\
\text { Reservations }\end{array}$ & cost \\
\hline 1 & All & $\begin{array}{l}\text { TRU content of } \\
\text { the waste } \\
\text { package. }\end{array}$ & $\begin{array}{l}\text { Dose rate, NDA, } \\
\text { measurement of gamma } \\
\text { and neutron emissions. } \\
\text { Sampling and analysis. }\end{array}$ & \multirow{2}{*}{$\begin{array}{l}\text { Conversion of } \\
\text { measured dose } \\
\text { rate and/or } \\
\text { gamma } \\
\text { emissions and } \\
\text { neutrons require } \\
\text { application of } \\
\text { estimated ratios } \\
\text { and generic dose } \\
\text { to curie } \\
\text { conversions. } \\
\text { Laboratory } \\
\text { analysis is costly } \\
\text { and will result in } \\
\text { dose to samplers } \\
\text { and analysts. } \\
\text { WHC (1996 a,b), } \\
\text { WHC (1996 f) }\end{array}$} & \\
\hline 2 & All & $\begin{array}{l}\text { Radionuclide } \\
\text { COC content of } \\
\text { waste package. }\end{array}$ & $\begin{array}{l}\text { Measurement of dose } \\
\text { rate, gamma emission } \\
\text { rates. Sampling and } \\
\text { laboratory analysis. }\end{array}$ & & \\
\hline 3,4 & $1-20$ & $\begin{array}{l}\text { TC metals } \\
\text { concentrations }\end{array}$ & $\begin{array}{l}\text { Process knowledge } \\
\text { coupled with scaling } \\
\text { factor. Laboratory } \\
\text { analysis. }\end{array}$ & $\begin{array}{l}\text { Scaling factors } \\
\text { will provide } \\
\text { conservative } \\
\text { results. } \\
\text { Laboratory } \\
\text { analysis is costly } \\
\text { and will result in } \\
\text { dose to samplers } \\
\text { and analysts }\end{array}$ & \\
\hline
\end{tabular}

Table 3-5 defines the analytical performance requirements for the data that need to be collected to resolve the DSs that require measurements. These performance requirements include the PQL and precision and accuracy requirements for each of the cocs. 
HNF-6273

Rev. 0

Table 3-5. Analytical Performance Requirements.

\begin{tabular}{|c|c|c|c|c|c|c|}
\hline $\begin{array}{l}\text { lndivioual } \\
\text { Wasto } \\
\text { stream }\end{array}$ & arm & 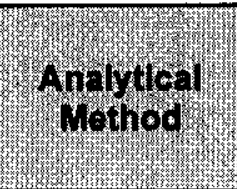 & $\begin{array}{l}\text { Proliming } \\
\text { Monon }\end{array}$ & 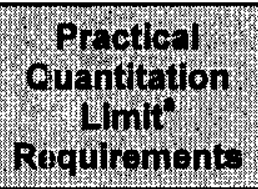 & Prociston & $\begin{array}{l}\text { Accuracy } \\
\text { Requ }\end{array}$ \\
\hline All & TRU COCs & $\begin{array}{l}\text { Dose rate, } \\
\text { gamma } \\
\text { spectroscopy } \\
\text { for Cs-137 for } \\
\text { curie } \\
\text { conversion of } \\
\text { TRU COC } \\
\text { radionuclides. }\end{array}$ & $100 \mathrm{nCi} / \mathrm{g}$ & TBD & TBD & TBD \\
\hline All & $\begin{array}{l}\text { Radiological } \\
\text { COCs }\end{array}$ & $\begin{array}{l}\text { Dose rate, } \\
\text { gamma } \\
\text { spectroscopy } \\
\text { for Cs-137 for } \\
\text { curie } \\
\text { conversion of } \\
\text { TRU COC } \\
\text { radionuclides. }\end{array}$ & $\begin{array}{l}\text { ERDF } \\
\text { radiological } \\
\text { WAC. }\end{array}$ & TBD & TBD & TBD \\
\hline
\end{tabular}

The PQLs are the appropriate limits in most cases. If the action levels are below the PQLs, minimum detection limits apply instead of the PQLs.

TBD = To be determined based on final selection of instruments. To be addressed in the SAP. PQLs, accuracy, and precision for any selected instrument and associated method or procedure must be adequate to meet the preliminary action levels for each WS. 
HNF-6273

Rev. 0 


\subsection{STEP 4 - DEFINE THE BOUNDARIES OF THE STUDY}

The primary objective of DQO Step 4 is to identify the geographic (spatial) and temporal boundaries of the facility under investigation, as well as practical constraints (e.g., hindrances or obstacles) that must be taken into consideration in the sampling design. Implementing this step ensures that the sampling design will result in the collection of data that accurately reflect the true condition of the facility.

\subsection{GEOGRAPHIC BOUNDARIES}

Prior to defining the boundaries of the facility, it is first necessary to clearly define the populations of interest that apply for each DS (Table 4-1). Table 4-1 clearly defines the attributes that make up each population of interest by stating them in a way that makes the focus of the study unambiguous.

Table 4-1. Characteristics that Define the Population of Interest.

\begin{tabular}{|c|c|c|c|c|c|}
\hline wsy & Dst & Populaton or lnerost & Characteristics & 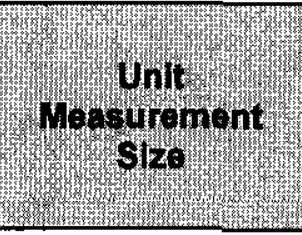 & $\begin{array}{l}\text { Total Number of } \\
\text { Potential } \\
\text { Moasurement Units } \\
\text { Within the } \\
\text { Population }\end{array}$ \\
\hline $\begin{array}{l}11- \\
14\end{array}$ & 1 & $\begin{array}{l}\text { Debris that is pressure } \\
\text { washed prior to removal } \\
\text { from the basins must be } \\
\text { characterized for TRU. }\end{array}$ & $\begin{array}{l}\text { TRU contaminants } \\
\text { on debris }\end{array}$ & $\begin{array}{l}\text { Bags, Barrels, } \\
\text { Boxes, Canisters, } \\
\text { Racks }\end{array}$ & $\begin{array}{l}\text { Variable and } \\
\text { unknown except } \\
\text { canisters and racks } \\
\text { TBD }\end{array}$ \\
\hline $1-20$ & 2 & $\begin{array}{l}\text { All debris is assumed to be } \\
\text { radiologically } \\
\text { contaminated. }\end{array}$ & $\begin{array}{l}\text { Radionuclide } \\
\text { contaminants on } \\
\text { debris }\end{array}$ & $\begin{array}{l}\text { Bags, Barrels, } \\
\text { Boxes, Canisters, } \\
\text { Racks }\end{array}$ & $\begin{array}{l}\text { Variable and } \\
\text { unknown except } \\
\text { canisters and racks }\end{array}$ \\
\hline 1 & $3 a$ & $\begin{array}{l}\text { Painted material is } \\
\text { assumed to be a mixed } \\
\text { waste, due to the presence } \\
\text { of TC constituents. All } \\
\text { painted debris will be } \\
\text { macro-encapsulated. }\end{array}$ & $\begin{array}{l}\text { TC constituents on } \\
\text { painted debris }\end{array}$ & $\begin{array}{l}\text { No measurements } \\
\text { planned }\end{array}$ & $\begin{array}{l}\text { No measurements } \\
\text { planned }\end{array}$ \\
\hline $\begin{array}{l}16- \\
20\end{array}$ & $3 b$ & $\begin{array}{l}\text { Building materials will be } \\
\text { inspected for the presence } \\
\text { of asbestos. }\end{array}$ & $\begin{array}{l}\text { Asbestos fiber } \\
\text { content }\end{array}$ & $\begin{array}{l}\text { Visual inspection } \\
\text { of entire material }\end{array}$ & $\begin{array}{l}\text { Entire material is } \\
\text { considered, no } \\
\text { subdivision }\end{array}$ \\
\hline $\begin{array}{c}1,2,1 \\
1,12 \\
13\end{array}$ & 4 & $\begin{array}{l}\text { Materials macro- } \\
\text { encapsulated in response } \\
\text { to PSQ \#3 concerns will be } \\
\text { considered to be in } \\
\text { compliance with LDR. }\end{array}$ & $\begin{array}{l}\text { TC constituents, } \\
\text { organics on painted } \\
\text { debris surfaces }\end{array}$ & $\begin{array}{l}\text { No measurements } \\
\text { planned }\end{array}$ & $\begin{array}{l}\text { No measurements } \\
\text { planned }\end{array}$ \\
\hline
\end{tabular}


Rev. 0

Table 4-2 identifies the geographic areas that apply to each DS. The term "geographic area" refers to the dimensions of the facility under investigation or the dimensions of the specific rooms or sections of the facility under investigation.

Table 4-2. Geographic Areas of Investigation.

\begin{tabular}{|c|c|}
\hline ost & Coographic Areas of investigation \\
\hline All & $\begin{array}{l}\text { The geographic areas of investigation include the structures that house the KE and KW } \\
\text { Basins, as discussed in the ROD as well as the IWTS. }\end{array}$ \\
\hline
\end{tabular}

Table 4-3 defines the zones or materials within the facility under investigation that have certain homogeneous characteristics. These zones or materials are identified by using existing information to segregate the elements of the population into subsets that exhibit relatively homogeneous characteristics, such as types of contaminants. This distinction reduces the overall complexity of the problem by segregating the facility or materials into more manageable pieces. 
HNF-6273

Rev. 0

Table 4-3. Strata with Homogeneous* Characteristics.

\begin{tabular}{|c|c|c|c|c|}
\hline Went & Dst & populaton of lintorout & Strata & $\begin{array}{l}\text { Homogeneous } \\
\text { Characterstic Logic }\end{array}$ \\
\hline 2 & 3 & $\begin{array}{l}\text { Rags Contaminated with } \\
\text { Stripped paint waste }\end{array}$ & $\begin{array}{l}\text { Stripped paint will be } \\
\text { collected on rags }\end{array}$ & $\begin{array}{l}\text { This will consist of paint chips } \\
\text { and Citristrip on rags/gloves. } \\
\text { The paint was homogeneous } \\
\text { on the applied surface and } \\
\text { would be comprised of similar } \\
\text { constituents. Using } \\
\text { calculations to cover the area } \\
\text { stripped versus the weight of } \\
\text { the rags, the total paint on } \\
\text { the rags will be estimated. }\end{array}$ \\
\hline 3 & $\begin{array}{c}2,3 \\
4\end{array}$ & Lead shielding & $\begin{array}{l}\text { Metal surface and } \\
\text { content }\end{array}$ & $\begin{array}{l}\text { Shielding will be of similar } \\
\text { construction; high lead } \\
\text { content }\end{array}$ \\
\hline $\begin{array}{c}1-2 \\
11-13\end{array}$ & 4 & Materials for which LDRs apply & Painted surfaces & $\begin{array}{l}\text { Paint is relatively uniformly } \\
\text { applied to surfaces } \\
\text { composed of similar } \\
\text { constituents, and the depth of } \\
\text { the coating is relatively } \\
\text { uniform. }\end{array}$ \\
\hline 14 & 3 & $\begin{array}{l}\text { Canister surfaces and all } \\
\text { nonporous material from under } \\
\text { water after pressure washing }\end{array}$ & Surface of metal & $\begin{array}{l}\text { The metal surface should be } \\
\text { homogeneous after washing. }\end{array}$ \\
\hline 15 & 2,3 & $\mathrm{IX}$ resins & IXMs & $\begin{array}{l}\text { The water is recirculated } \\
\text { making the constituents } \\
\text { relatively uniformly mixed and } \\
\text { deposited on the resin. } \\
\text { Water flow is monitored } \\
\text { during their service life. }\end{array}$ \\
\hline 16 & 2,3 & $\begin{array}{l}\text { Asbestos fiber content, } \\
\text { radiological constituents }\end{array}$ & Surfaces of materials & $\begin{array}{l}\text { The asbestos is typically } \\
\text { mixed in the tile material or } \\
\text { on the applied dried } \\
\text { adhesives, therefore, the } \\
\text { manufacturing and usage } \\
\text { result in relative } \\
\text { homogeneity. }\end{array}$ \\
\hline
\end{tabular}

* The term is used to mean relatively similar waste. None of the waste in these documents exhibits true homogeneity.

Table 4-4 identifies the spatial scale for decision making that may apply to each DS. The spatial scale for decision making identifies each decision unit, which is the smallest area or volumetric unit for which each decision applies. 
Rev. 0

Table 4-4. Spatial Scale for Decision Making.

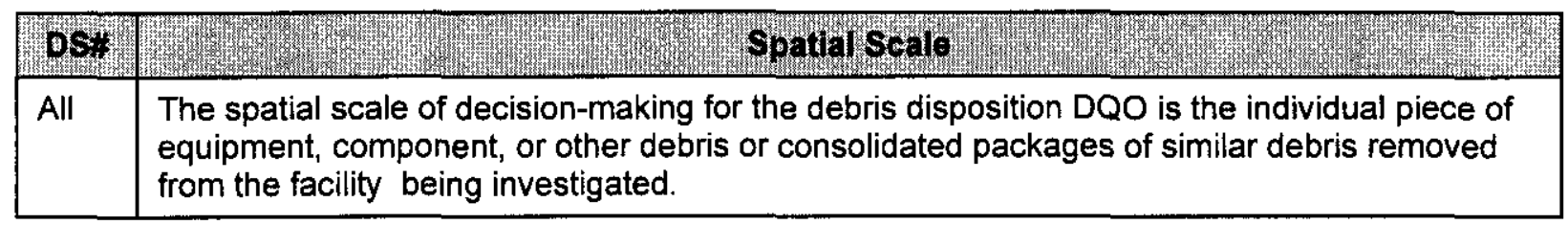

\subsection{TEMPORAL BOUNDARIES}

Table 4-5 defines the temporal scale for decision making. A temporal scale for decision making may be necessary for certain types of studies.

Table 4-5. Temporal Scale for Decision Making.

\begin{tabular}{|c|l|c|}
\hline DSH & \multicolumn{1}{|c|}{ Tomporal scale } \\
\hline All & $\begin{array}{l}\text { The decisions identified in this DQO apply to the removal of all debris covered by the ROD } \\
\text { during this initial phase of K-Basin remedial activities. The decisions may or may not be } \\
\text { appropriate for later debris removal activities, particularly for those associated with D\&D of } \\
\text { structures not covered by this ROD. }\end{array}$ \\
\hline
\end{tabular}

\subsection{PRACTICAL CONSTRAINTS}

Access to the basins may require the removal of internal walls or other portions of the $\mathrm{K}$-Basin structures. Sludge must be removed from debris prior to disposal. Sludge within the basins will create visibility problems during retrieval and washing of the debris from within the basins. Sampling and analysis of paint samples and other potential COCs from multiple objects would not be cost-effective, when considering the small potential volume of these materials. The large number of debris and the difficulty associated with collecting representative samples from the variety of matrices supports the use of field radiological measurements over sampling and laboratory-based analysis for radionuclides for each item. 
Rev. 0

\subsection{STEP 5 - DEVELOP A DECISION RULE}

The purpose of DQO Step 5 is to combine information provided in DQO Steps 1 through 4 with a parameter of interest (e.g., mean, median, or percentile) and an action level to provide a concise description of what action will be taken based on the results of the data collected. The parameter of interest specifies the characteristic or attribute that the decision-maker would like to know about the population, while the action level is a threshold value of the parameter of interest that provides the criterion for choosing between alternative actions.

\subsection{STATISTICAL PARAMETER OF INTEREST}

For each COC, Table 5-1 identifies the corresponding statistical parameter of interest (e.g., mean and upper $95^{\text {th }}$ percent confidence interval).

Table 5-1. Statistical Parameter of Interest.

\begin{tabular}{|c|c|c|}
\hline 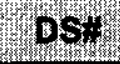 & Becision Statoment & Paramoter of lncerest \\
\hline 1 & $\begin{array}{l}\text { Determine if the potentially contaminated debris } \\
\text { materials exceed the radiological contamination } \\
\text { limits for TRU and, therefore, are not eligible for } \\
\text { disposal at the ERDF. }\end{array}$ & $\begin{array}{l}\text { The norm will be the maximum } \\
\text { activity for } \mathrm{Cs}-137 \text {. Options will be } \\
\text { developed as needed. }\end{array}$ \\
\hline 2 & $\begin{array}{l}\text { Determine if the radiological activity of the debris } \\
\text { exceeds the ERDF WAC (BHI 1998) limits. }\end{array}$ & $\begin{array}{l}\text { The norm will be the maximum } \\
\text { activity for Cs- } 137 \text {. Options will be } \\
\text { developed as needed. }\end{array}$ \\
\hline 3 & $\begin{array}{l}\text { Determine if the material designates as dangerous, } \\
\text { TC or asbestos waste. }\end{array}$ & \multirow{2}{*}{$\begin{array}{l}\text { Process knowledge, or analytical } \\
\text { results: Single sample } \\
\text { concentrations. }\end{array}$} \\
\hline $3 a$ & $\begin{array}{l}\text { Determine if the TC waste codes or state } \\
\text { dangerous/toxic codes apply to the material. }\end{array}$ & \\
\hline $3 b$ & $\begin{array}{l}\text { Determine if the material is regulated due to } \\
\text { asbestos content. }\end{array}$ & $\begin{array}{l}\text { Observed single sample fiber count } \\
\text { or process knowledge. }\end{array}$ \\
\hline 4 & Determine if LDRs impose treatment for material. & $\begin{array}{l}\text { Process knowledge, material safety } \\
\text { data sheet data, any analytical } \\
\text { sample result. }\end{array}$ \\
\hline
\end{tabular}

\subsection{FINAL ACTION LEVELS}

Table 5-2 lists the final action level for each DS and COC. 
Rev. 0

Table 5-2. Final Action Level for the Decision.

\begin{tabular}{|c|c|c|}
\hline $\mathrm{BSt}$ & (1) & Actor Level \\
\hline 1 & $\begin{array}{l}\text { Estimated concentration of TRU COC } \\
\text { radionuclides. }\end{array}$ & $100 \mathrm{nCi} / \mathrm{g}$ TRU radionuclides in waste. \\
\hline 2 & $\begin{array}{l}\text { Estimated concentration of all } \\
\text { radiological COCs. }\end{array}$ & $\begin{array}{l}\text { Disposal facility WAC limits. Units of } \\
\mathrm{Ci} / \mathrm{m}^{3} \text { on a per waste package basis. }\end{array}$ \\
\hline \multirow[t]{9}{*}{$3 a$} & 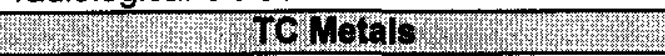 & $=\quad$ TGL P Action Lovels (mgL) \\
\hline & Arsenic & 5.0 \\
\hline & Barium & 100 \\
\hline & Cadmium & 1.0 \\
\hline & Chromium & 5.0 \\
\hline & Lead & 5.0 \\
\hline & Mercury & 0.2 \\
\hline & Selenium & 1.0 \\
\hline & Silver & 5.0 \\
\hline $3 \mathrm{~b}$ & Asbestos & $<1 \%$ asbestos by volume \\
\hline 4 & LDR constituents & See ERDF WAC (BHI 1998) \\
\hline
\end{tabular}

"If TC criteria are exceeded, evaluate waste for potential underlying hazardous constituents and evaluate any underlying hazardous constituents against the limits in the universal treatment standards criteria of 40 CFR 268.48 .

\subsection{DECISION RULES}

Table 5-3 combines the parameter of interest, scale for decision making, action levels, and alternative actions into separate "IF...THEN..." statements that are referred to as decision rules (or DRs). Each DS identified in Table 2-3 that requires additional information (Tables 3-1 and 3-1a) has one or more DRs associated with it.

Table 5-3. Decision Rules. (2 Pages)

\begin{tabular}{|l|l|}
\hline 102 & Doclsion Rulo \\
\hline 1 & $\begin{array}{l}\text { If the estimated TRU COCs in the waste do not exceed } 100 \mathrm{nCi} / \mathrm{g} \text {, then the waste will be } \\
\text { evaluated per DRs \#2, 3, and } 4 \text { for disposal at ERDF. } \\
\text { If the estimated TRU COCs in the waste exceeds } 100 \mathrm{nCi} / \mathrm{g} \text {, then the waste will not be sent to } \\
\text { ERDF. }\end{array}$ \\
\hline 2 & $\begin{array}{l}\text { If the estimated radionuclide COCs in the waste do not exceed the radionuclide ERDF WAC } \\
(\mathrm{BHI} 1998)\left(\mathrm{Ci} / \mathrm{m}^{3}\right), \text { then the waste will be evaluated per DRs \# 3, and } 4 . \\
\text { If the in estimated radionuclide COCs in the waste exceeds the radionuclide ERDF WAC } \\
(\mathrm{BHI} 1998)\left(\mathrm{Ci} / \mathrm{m}^{3}\right), \text { then the waste will be evaluated on a case-by-case basis and may not be } \\
\text { sent to ERDF. }\end{array}$ \\
\hline
\end{tabular}


HNF-6273

Rev. 0

Table 5-3. Decision Rules. (2 Pages)

\begin{tabular}{|l|l|l|}
\hline DR & (1) & Decision Rulle \\
\hline 3 & $\begin{array}{l}\text { If process knowledge, or single sample concentrations of the detected analytical value, or the } \\
\text { observed fiber count indicates that the materials do not designate as TC or exceed ERDF } \\
\text { WAC (BHI 1998), then they will be packaged for disposal at the ERDF as LLW. Waste which } \\
\text { designates only as Washington State dangerous will not require treatment before disposal. } \\
\text { If process knowledge, or single sample concentrations of the detected analytical value, or the } \\
\text { observed fiber count indicates that the materials designate as TC, state dangerous EHW, or } \\
\text { exceed ERDF WAC (BHI 1998), then they will be treated through macro-encapsulation and } \\
\text { disposed of at ERDF. }\end{array}$ \\
\hline 4 & $\begin{array}{l}\text { If process knowledge or any detected analytical sample value dictates LDR imposed } \\
\text { treatment, the materials will be treated with macro-encapsulation and disposed at ERDF. } \\
\text { If process knowledge or none of the detected analytical sample values dictate LDR imposed } \\
\text { treatment of the materials, the debris will be disposed in ERDF without additional treatment. }\end{array}$ \\
\hline
\end{tabular}


HNF-6273

Rev. 0 
HNF-6273

Rev. 0

\subsection{STEP 6 - SPECIFY TOLERABLE LIMITS ON DECISION ERRORS}

The sampling design for the materials is performed in two stages. In Stage 1, facility or historical radiological sample data (99-SNF/CJS-024, PNNL 1999, Huisingh 1997, Numatec 1996, WHC 1997a, WHC 1997b, WHC 1996g, WHC 1990) will be used to establish the radionuclide/isotopic distribution of radiological COCs. The radionuclide distributions will be established for each waste WS and subsequently used to estimate the content of COCs other than cesium-137. The cesium-137 content of the waste will be estimated using a variety of instruments, including portable radiation detectors (WHC 1996a, WHC 1996b). Nondestructive analysis (gamma spectroscopy, neutron counting) equipment as described in previous characterizations (PNNL 1999, WHC 1996f) may be used. During Stage 2, K-Basin staff will use the correlation when evaluating data from radiological NDA, dose rate or gamma surveys to estimate isotopic inventories for waste shipments.

The purpose of DQO Step 6 is to develop tolerable error limits. To quantify error limits, statistical procedures may be used when sampling and analyzing the data. Because the goal for Stage 1 sampling is to determine radionuclide/isotopic distributions, suspect contaminated areas either have been, or will be, preferentially sampled. This design is judgmental, so the quantification of error limits is not feasible. Likewise, the radionuclide sampling for Stage 2 will also be preferentially determined, based on a qualitative probability of contamination.

It should be noted that the sampling design for certain material/waste accumulations may benefit from a statistical derivation. These situations are not expected, but if they arise, the statistical sampling approach should be developed on a case-by case basis. In those cases, the development of error tolerances and optimization of the sampling design will follow Steps 6 and 7 in the remediation DQO workbook template (http://www.hanford.gov/dqo/index.html).

\subsection{STATISTICAL VS. JUDGMENTAL DESIGN}

\subsubsection{Radioactive Waste}

One of the primary objectives normally accomplished in DQO Step 6 is the selection of a statistical or judgmental sample design. Statistically-based sampling for radiological COCs will not be used because of the cost associated with sampling and difficulty of sampling debris matrices. Each waste container will be either surveyed or contain surveyed waste. An estimated $\mathrm{COC}$ inventory for that waste container will be derived from measurements. Therefore, the sample design is judgmentally developed for the materials components that will ultimately be placed in the shipping container. Specific radiological survey requirements, procedures, and dose-to-curie relationships will be discussed in the subsequent sampling and analysis plan supporting K-Basin waste characterization. 
Rev. 0

\subsubsection{Potentially Chemically Contaminated Waste}

No sampling for chemical constituents is currently planned for most of the WSs. The basin water flowing into the IXM is sampled routinely and the radionuclide load estimated (WHC 1996e). Based on the analyses described previously in this document, the IXMs are presumed to be suitable for disposal as debris without further sampling. The WSs, other than IXM, that pose a potential for chemical contamination are:

- Dried paint, painted debris (WS\#1)

- Rags Contaminated with Stripped paint waste (WS\#2)

- Lead shielding (WS\#3)

- Material that has contacted the sludge from the K Basin. (WS\#12, 13, 14)

Waste streams \# 1, 2, and 3 will be encapsulated; therefore, no sampling is needed to designate those wastes. Waste streams \#12-14 will be pressure washed to remove potential TC metals and PCBs. Previous studies indicate that washing removes the metals and PCBs on debris that has been in contact with the sludge (WHC 1996d). Calculations supporting these studies are presented in Appendix D, which was part of a previous profile used for disposal at the CWC. 
Rev. 0

\subsection{STEP 7 - OPTIMIZE THE DESIGN}

\subsection{MATERIAL (COMPONENT) CATEGORIES}

Table 7-1 lists the material components to be characterized and the significance of the survey and sampling approach. Note that section 6.0 provides the logic for selection of a judgmental design and provides the reasons that chemical sampling will not be required. No survey or sampling is required, therefore, for DS \#3 or 4 .

Table 7-1. Material (Component) Categories.

\begin{tabular}{|c|c|c|}
\hline bost & Materlat (Componen tr) / Cotogoros & survoy Sampling significanco \\
\hline 1 & All WSs & $\begin{array}{l}\text { Determine if the waste package contains } \\
\text { TRU COCs at concentrations greater } \\
\text { than } 100 \mathrm{nCi} / \mathrm{g} \text {. }\end{array}$ \\
\hline 2 & All WSs & $\begin{array}{l}\text { Determine if the waste package contains } \\
\text { radionuclide COCs at concentrations } \\
\text { greater than ERDF WAC (BHI 1998). }\end{array}$ \\
\hline
\end{tabular}

\subsection{SURVEYISAMPLING DESIGN ALTERNATIVES}

Table 7-2 identifies, describes, and compares the survey/sampling design alternatives for the material (component) categories, including costs.

\subsection{SELECTION OF THE SURVEYISAMPLING DESIGN}

The process of determining the sampling requirements and selecting the most resource effective design is presented along with a recommended design. Tables 7-2 and 7-3 discuss the analytical methods and design alternatives. Table 7-4 provides the design summary. 
Rev. 0

Table 7-2. Waste Designation Survey/Sampling Methods. (2 pages)

\begin{tabular}{|c|c|c|c|}
\hline 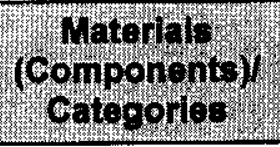 & Survoylsampling & moscripton & $\begin{array}{l}\text { Rolatyo } \\
\text { Cost }\end{array}$ \\
\hline \multirow[t]{4}{*}{$\begin{array}{l}\text { All debris WSs } \\
\text { except IXMs. }\end{array}$} & \multirow[t]{4}{*}{$\begin{array}{l}\text { Dose rate, portable } \\
\text { gamma spectroscopy, } \\
\text { NDA. }\end{array}$} & $\begin{array}{l}\text { Once the waste is packaged in a } \\
\text { plastic bag barrel or box, measurement } \\
\text { of gamma emitting radionuclides will } \\
\text { occur and an estimate of the Cs- } 137 \\
\text { content of the waste package will be } \\
\text { obtained. There are three main } \\
\text { approaches: }\end{array}$ & \\
\hline & & $\begin{array}{l}\text { 1. The dose rate (mR/hr) may be } \\
\text { estimated and published dose to curie } \\
\text { relationships may be used (WHC } \\
\text { 1997a, WHC 1997b) to estimate Cs- } \\
\text { 137. Provides data with the least } \\
\text { accuracy and precision of the three } \\
\text { approaches. }\end{array}$ & Low \\
\hline & & $\begin{array}{l}\text { 2. Portable gamma spectroscopy } \\
\text { using shielded and/or collimated } \\
\text { portable instruments. } \\
\text { Can provide accurate data if properly } \\
\text { calibrated to match waste types. Only } \\
\text { measures gamma emitters, typically } \\
\text { not adequate sensitivity to measure } \\
\text { low energy gamma's such as emitted } \\
\text { by Am-241. }\end{array}$ & Medium \\
\hline & & $\begin{array}{l}\text { 3. Perform NDA on a bag barrel or } \\
\text { box of waste. NDA usually consists of } \\
\text { a segmented gamma scan capability } \\
\text { that performs multiple gamma } \\
\text { spectroscopy measurements of a } \\
\text { barrel or box on specific segments of } \\
\text { the waste. NDA also usually includes } \\
\text { measurement of passive neutron flux. } \\
\text { Commercial NDA units also may have } \\
\text { the capability of performing both } \\
\text { passive and active neutron } \\
\text { measurements. } \\
\text { Can provide accurate data if properly } \\
\text { calibrated to match waste types. } \\
\text { Specifically designed to measure low } \\
\text { energy gamma emissions, can provide } \\
\text { estimate of TRU content based on } \\
\text { neutron counts. }\end{array}$ & High \\
\hline
\end{tabular}


Table 7-2. Waste Designation Survey/Sampling Methods. (2 pages)

\begin{tabular}{|c|c|c|c|}
\hline 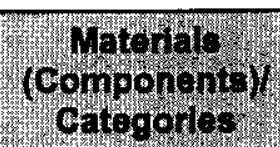 & suroysampling & Descrpton & $\begin{array}{l}\text { Relatve } \\
\text { Cost }\end{array}$ \\
\hline $\begin{array}{l}\text { All debris WSs } \\
\text { except IXMs } \\
\text { (cont.) }\end{array}$ & $\begin{array}{l}\text { Laboratory analysis of } \\
\text { samples to determine } \\
\text { TRU and radiological } \\
\text { COC content. }\end{array}$ & $\begin{array}{l}\text { Waste sampling and radiochemical } \\
\text { analysis. Generally provides most } \\
\text { accurate measurement of } \\
\text { radionuclides in the sample because } \\
\text { geometry and matrix effects are largely } \\
\text { eliminated in the laboratory. However, } \\
\text { obtaining a sample that is } \\
\text { representative of the waste may be } \\
\text { difficult depending on the distribution of } \\
\text { radionuclides on the waste and the } \\
\text { difficulty in obtaining a sample from the } \\
\text { waste. Can measure the gamma } \\
\text { emitting COCs as well as pure alpha } \\
\text { and pure beta emitters (Huisingh } 1997 \text {, } \\
\text { WHC 1996g). }\end{array}$ & High Cost \\
\hline IXM & $\begin{array}{l}\text { Sampling of fuel basin } \\
\text { water that flows through } \\
\text { the IXM. }\end{array}$ & $\begin{array}{l}\text { The radionuclide COC content of IXMs } \\
\text { will be estimated based on historical } \\
\text { radiochemistry results of the basin } \\
\text { water, measured flow rates and time in } \\
\text { service (WHC 1996e, WHC 1996f). }\end{array}$ & $\begin{array}{l}\text { No additional } \\
\text { cost. Part of } \\
\text { current } \\
\text { operations. }\end{array}$ \\
\hline
\end{tabular}

Table 7-3. Key Features of Sampling Design. (2 pages)

\begin{tabular}{|c|c|c|c|}
\hline (Componons)) & Surpy Sample colloctlon & Key Featuros of Dos lgn & $\begin{array}{l}\text { Basle ror } \\
\text { surveysampling } \\
\text { besign }\end{array}$ \\
\hline $\begin{array}{l}\text { All debris WSs } \\
\text { except fuel } \\
\text { canisters and } \\
\text { IXMs. }\end{array}$ & $\begin{array}{l}\text { Obtain an estimate of the Cs- } \\
137 \text { activity for a container of } \\
\text { waste and apply historical } \\
\text { cesium to radionuclide ratios } \\
\text { to estimate the concentration } \\
\text { of COCs other than cesium. } \\
\text { Cs- } 137 \text { activity in the waste } \\
\text { may be obtained using } \\
\text { measured dose rates, NDA, } \\
\text { and/or gamma spectroscopy. } \\
\text { If the estimated TRU content of } \\
\text { the waste exceeds } 100 \text { nCi/g, } \\
\text { the waste may be subjected to } \\
\text { additional measurements. } \\
\text { Additional measurements may } \\
\text { include gamma spectroscopy } \\
\text { or neutron emission. } \\
\text { Judgmental samples of the } \\
\text { waste may be obtained and } \\
\text { subjected to laboratory } \\
\text { radiochemistry analysis to } \\
\text { confirm the estimated ratios of } \\
\text { COC radioniclides to Cs-137. }\end{array}$ & $\begin{array}{l}\text { Each container of debris will } \\
\text { be surveyed for gamma } \\
\text { and/or neutron emitters using } \\
\text { one or more of the } \\
\text { survey/sample collection } \\
\text { methodologies. } \\
\text { The radionuclide COC } \\
\text { content of the waste } \\
\text { (including TRU) will be } \\
\text { estimated by applying } \\
\text { historical radionuclide ratios } \\
\text { to the measured Cs-137 } \\
\text { activity (Appendix B). } \\
\text { In some instances, the Cs- } \\
137, \text { Am-241, and Pu- } \\
239 / 240, \text { and other } \\
\text { radionuclides will be } \\
\text { estimated based on analytical } \\
\text { data specific to the WS } \\
\text { (Huisingh } 1997, \text { WHC } 1997 \mathrm{~b} \text {, } \\
\text { WHC } 1996 \mathrm{~g} \text { ). } \\
\text { These actions will provide a } \\
\text { more accurate estimation of } \\
\text { TRU content when needed. }\end{array}$ & $\begin{array}{l}\text { Consistent with } \\
\text { previous practice at } \\
\text { K-Basins and will } \\
\text { provide an adequate, } \\
\text { cost-effective estimate } \\
\text { of radionuclide content } \\
\text { of waste. Boxes or } \\
\text { barrels containing } \\
\text { multiple bags may be } \\
\text { measured in bulk or } \\
\text { may be estimated by } \\
\text { summing the results of } \\
\text { individual bags in the } \\
\text { larger container. } \\
\text { If the waste is super } \\
\text { compacted, the final } \\
\text { concentration of } \\
\text { radionuclides in the } \\
\text { compacted waste as } \\
\text { packaged will be } \\
\text { estimated from } \\
\text { compaction ratios. }\end{array}$ \\
\hline
\end{tabular}


Table 7-3. Key Features of Sampling Design. (2 pages) HNF-6273

\begin{tabular}{|c|c|c|c|}
\hline $\begin{array}{l}\text { Waterlal } \\
\text { (Componentis) } \\
\text { Catogories }\end{array}$ & survy sample colled on & Key Fortures of oposign & $\begin{array}{l}\text { Easis for } \\
\text { Surveysampling } \\
\text { Design }\end{array}$ \\
\hline Fuel Canisters & $\begin{array}{l}\text { Obtain an estimate of the Cs- } \\
137 \text { activity for a fuel canister } \\
\text { and apply historical cesium to } \\
\text { radionuclide ratios to estimate } \\
\text { the concentration of COCs } \\
\text { other than cesium. Cs-137 } \\
\text { activity in the waste may be } \\
\text { obtained using measured dose } \\
\text { rates, NDA, and/or gamma } \\
\text { spectroscopy. } \\
\text { If the estimated TRU content of } \\
\text { the waste exceeds } 100 \text { nCi/g, } \\
\text { the fuel canister may be } \\
\text { subjected to additional } \\
\text { measurements. Additional } \\
\text { measurements may include } \\
\text { gamma spectroscopy or } \\
\text { neutron emission. Judgmental } \\
\text { samples of the fuel canister } \\
\text { may be obtained and } \\
\text { subjected to laboratory } \\
\text { radiochemistry analysis to } \\
\text { confirm the estimated ratios of } \\
\text { COC radionuclides to Cs- } 137 \text {. }\end{array}$ & $\begin{array}{l}\text { Fuel canisters will be } \\
\text { surveyed for gamma and/or } \\
\text { neutron emitters using one or } \\
\text { more of the survey/sample } \\
\text { collection methodologies. } \\
\text { The radionuclide COC } \\
\text { content of the fuel canister } \\
\text { (including TRU) will be } \\
\text { estimated by applying } \\
\text { historical radionuclide ratios } \\
\text { to the measured Cs-137 } \\
\text { activity. } \\
\text { In some instances, the Cs- } \\
137 \text {, Am-241, and Pu- } \\
239 / 240, \text { and other } \\
\text { radionuclides will be } \\
\text { estimated based on analytical } \\
\text { data specific to the fuel } \\
\text { canister WS (WHC } 1997 \mathrm{~b}, \\
\text { WHC } 1996 f) \text {. } \\
\text { These actions will provide a } \\
\text { more accurate estimation of } \\
\text { TRU content when needed. }\end{array}$ & $\begin{array}{l}\text { Consistent with } \\
\text { previous practice at } \\
\text { K Basins and will } \\
\text { provide an adequate, } \\
\text { cost-effective estimate } \\
\text { of radionuclide content } \\
\text { of fuel canisters. } \\
\text { Boxes or barrels } \\
\text { containing fuel } \\
\text { canisters may be } \\
\text { estimated by summing } \\
\text { the results of fuel } \\
\text { canister surveys in the } \\
\text { larger container. } \\
\text { If fuel canisters are } \\
\text { super-compacted, the } \\
\text { final concentration of } \\
\text { radionuclide in the } \\
\text { compacted canisters } \\
\text { as packaged will be } \\
\text { estimated from } \\
\text { compaction ratios. }\end{array}$ \\
\hline IXM & None & $\begin{array}{l}\text { The concentration of } \\
\text { radionuclides in the water of } \\
\text { the KW and KE Basins is } \\
\text { measured on a routine basis } \\
\text { (WHC, 1996e). The flow rate } \\
\text { of the basin water and the } \\
\text { time in service for the IXM are } \\
\text { also known. The } \\
\text { concentration of radionuclides } \\
\text { in the IXM waste package will } \\
\text { be estimated based on these } \\
\text { known values. (More detail } \\
\text { on this sampling program will } \\
\text { be provided in the SAP.) }\end{array}$ & $\begin{array}{l}\text { Consistent with } \\
\text { previous practice at } \\
\text { K Basins and will } \\
\text { provide adequate } \\
\text { estimate of } \\
\text { radionuclide content of } \\
\text { waste package. }\end{array}$ \\
\hline
\end{tabular}

\subsection{DESIGN SUMMARY}

Table 7-4 summarizes the selected sampling frequencies and locations. 
Rev. 0

Table 7-4. Summary of Sampling Frequencies and Locations. (2 Pages)

\begin{tabular}{|c|c|c|c|}
\hline$(\mathrm{Gompon})$ & 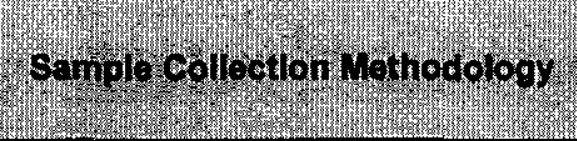 & $\begin{array}{l}\text { Samping } \\
\text { Frequency }\end{array}$ & Sampling \\
\hline $\begin{array}{l}\text { All WSs except fuel } \\
\text { canisters and IXMs }\end{array}$ & $\begin{array}{l}\text { Measurement of external dose rate, } \\
\text { NDA, gamma spectroscopy, or } \\
\text { sampling and laboratory analysis as } \\
\text { appropriate to determine TRU and } \\
\text { radiological COC content. }\end{array}$ & $\begin{array}{l}\text { Every bag barrel } \\
\text { or box of debris. }\end{array}$ & $\begin{array}{l}\text { Survey } \\
\text { measurements } \\
\text { will be } \\
\text { performed on } \\
\text { the waste. } \\
\text { Specific } \\
\text { locations will be } \\
\text { described in the } \\
\text { SAP. } \\
\text { Measurements } \\
\text { may be taken on } \\
\text { individual debris } \\
\text { items, on a } \\
\text { suitable } \\
\text { container of } \\
\text { debris. Survey } \\
\text { location will be } \\
\text { described in the } \\
\text { SAP and } \\
\text { associated } \\
\text { procedures. }\end{array}$ \\
\hline Fuel Canisters & $\begin{array}{l}\text { Measurement of external dose rate, } \\
\text { NDA, or gamma spectroscopy to } \\
\text { determine TRU and radiological } \\
\text { COC content. }\end{array}$ & $\begin{array}{l}\text { All fuel canisters } \\
\text { may be measured } \\
\text { individually or in } \\
\text { larger containers, } \\
\text { depending on final } \\
\text { survey } \\
\text { methodology. }\end{array}$ & $\begin{array}{l}\text { Survey } \\
\text { measurements } \\
\text { will be } \\
\text { performed on } \\
\text { the waste. } \\
\text { Specific } \\
\text { locations will be } \\
\text { described in the } \\
\text { SAP. } \\
\text { Measurements } \\
\text { may be taken on } \\
\text { individual debris } \\
\text { items, on a } \\
\text { suitable } \\
\text { container of } \\
\text { debris. Survey } \\
\text { location will be } \\
\text { described in the } \\
\text { SAP and } \\
\text { associated } \\
\text { procedures. }\end{array}$ \\
\hline
\end{tabular}




\section{HNF-6273}

Rev. 0

Table 7-4. Summary of Sampling Frequencies and Locations. (2 Pages)

\begin{tabular}{|c|c|c|c|}
\hline (Compony) & sumple collodion whododogy & Sraphing & $\begin{array}{l}\text { sampling } \\
\text { Lection }\end{array}$ \\
\hline IXM & None & $\begin{array}{l}\text { Radionuclide load } \\
\text { for each IXM will } \\
\text { be calculated } \\
\text { based on the } \\
\text { procedures on the } \\
\text { SNF Project Basin } \\
\text { Water Quality } \\
\text { Control Procedure } \\
\text { (SNF 2000) and } \\
\text { non exchange } \\
\text { module } \\
\text { characterization } \\
\text { plan (WHC } \\
\text { 1997a). }\end{array}$ & None \\
\hline
\end{tabular}




\subsection{REFERENCES}

40 CFR 61, "National Emissions Standards for Hazardous Air Pollutants," Code of Federal Regulations, as amended.

40 CFR 261, "Identification and Listing of Hazardous Waste," Code of Federal Regulations, as amended.

40 CFR 265, "Interim Status Standards for Owners and Operators of Hazardous Waste Treatment, Storage, and Disposal Facilities," Code of Federal Regulations, as amended.

40 CFR 268.48, "Land Disposal Restrictions," Code of Federal Regulations, as amended.

40 CFR 761, "Polychlorinated Biphenyls (PCBs)," Code of Federal Regulations, as amended.

99-SNF/CJS-024, "Facility Source Term Report", Interoffice Correspondence from P.G. Huntley to C. J. Slotemaker, dated March 29, 1999.

BHI, 1997, Supplemental Waste Acceptance Criteria for Bulk Shipments to the Environmental Restoration Disposal Facility, 0000X-DC-W0001, Rev. 1, Bechtel Hanford, Inc., Richland, Washington.

BHI, 1998, Environmental Restoration Disposal Facility Waste Acceptance Criteria, BHI-00139, Rev. 3, Bechtel Hanford, Inc., Richland, Washington.

Comprehensive Environmental Response, Compensation, and Liability Act of 1980, 42 USC 9601 et seq.

DeVanney, 1990, Characterization of Radioactive Waste at 100 Area, WHC-SD-NRRPT-005, Rev. 0, Westinghouse Hanford Company, Richland, Washington.

DOE-RL, 1998, Focused Feasibility Study for the K Basins Interim Remedial Action, DOE/RL-98-66, Rev. 0, U.S. Department of Energy, Richland Operation Office, Richland, Washington.

DOE-RL, 1999a, Remedial Design Report and Remedial Action Work Plan for the K Basins Interim Remedial Action, DOE/RL-99-89, Rev. 0, U.S. Department of Energy, Richland Operations Office, Richland, Washington.

DOE-RL, 1999b, 105 K-Basins 1998 Debris Report, DOE/RL-99-25, Rev. 0, U.S. Department of Energy, Richland Operations Office, Richland, Washington. 
EPA, 1997, Test Methods for Evaluating Solid, Waste Physical/Chemical Methods, SW-846, 3rd Edition, as amended by Updates I (July, 1992), IIA (August, 1993), IIB (January, 1995), and III (1997), U.S. Environmental Protection Agency, Washington, D.C.

EPA, DOE, and Ecology, 1999, Declaration of the Record of Decision for DOE Hanford 100 Area, 100-KR-2 Operable Unit, U.S. Department of Energy,

U.S. Environmental Protection Agency (EPA 541-R99-059), and Washington State Department of Ecology, Richland, Washington.

HNF, 1998, Hanford Site Solid Waste Acceptance Criteria, HNF-EP-0063, Rev. 5, Fluor Daniel Hanford, Richland, Washington.

Huisingh, J., 1997, "222-S Final Hanger Coupon Analysis and Rad Survey Reports", Don't Say It...Write It!, dated January 30, 1997.

Knox, J.M., 1997, K Basins Debris Inventory, HNF-SD-SNF-TI-052, Rev. 0, Duke Engineering and Services Hanford, Richland, Washington.

Numatec, 1996, "Analytical Report for K-Basin - FT6112", NHC-96-010, Letter from L. L. Lockrem to D. L. Rehn, Numatec Hanford Corporation, Richland, Washington.

PNNL, 1999, "PNNL Barrel Assayer Results", Letter from R. J. Arthur to D. L. Haggard, dated July 12, 1999, Pacific Northwest National Laboratory, Richland, Washington.

Resource Conservation and Recovery Act of 1976, 42 U.S.C 6901 et seq.

SNF Project, 2000, Basin Water Quality Control, OP-2-025-00, SNF Project Administrative Procedure, SNF Project, Richland, Washington

SNF Project, 1994, Remove Debris from Storage Basin for Disposal, SNF Operations Project Technical Procedure, OP-07,071, Rev. 0/E, SNF Project, Richland, Washington.

Toxic Substances Control Act of 1976, 15 U.S.C., et seq.

WAC-173-303, Dangerous Waste Criteria, Washington Administrative Code, as amended.

WHC, 1990, Characterization of Radioactive Waste at 100 N, WHC-SD-NR-RPT-005, Rev. 0, Westinghouse Hanford Company, Richland, Washington.

WHC, 1993, Hanford Site Solid Waste Acceptance Criteria, WHC-0063-4, Rev. 4, Westinghouse Hanford Company, Richland, Washington. 
WHC, 1996a, Basis for Dose Rate to Curie Assay Method, WHC-SD-WM-RPT-267, Rev. 0, Westinghouse Hanford Company, Richland, Washington.

WHC, 1996b, Procedure for Categorizing and Inventorying Waste in Standard Containers, WHC-SD-WM-PROC-020, Rev. 0, Westinghouse Hanford Company, Richland, Washington.

WHC, 1996c, "105 K East Basin Polychlorinated Biphenyls Spill Cleanup Plan", 9652094D, Letter from D.W. Siddoway to E. D. Sellers, dated June 14, 1996, Westinghouse Hanford Company, Richland, Washington.

WHC, 1996d, 105KE Basin PCB Wipe Sampling and Analysis, WHC-SD-SNF-EV-001, Rev. 0, ECN 189420, Westinghouse Hanford Company, Richland, Washington.

WHC, 1996e, Characterization Plan for Spent KE Basin Ion Exchange Modules, WHCSD-SNF-TI-039, Rev. 0, Westinghouse Hanford Company, Richland, Washington.

WHC, 1996f, Characterization of Empty Fuel Storage Canisters in 105 KE Basin, WHCSD-SNF-TI-019, Rev. 0, Westinghouse Hanford Company, Richland, Washington.

WHC, 1996g, "Analytical Report for K Basin Pipe - FT6021", 75745-FAST-96-050, Internal Memo from L. L. Lockrem to R. M. Jochen, dated June 5, 1996, Westinghouse Hanford Company, Richland, Washington.

WHC, 1997a, Characterization Plan for Spent KE Basin Ion Exchange Modules, WHC-SD-SNF-TI-039, Rev. 1, Westinghouse Hanford Company, Richland, Washington.

WHC, 1997b, 105-K Basin Material Design Basis Feed Description for Spent Nuclear Fuel Project Facilities, HNF-SD-SNF-TI-009, Rev. 1, Westinghouse Hanford Company, Richland, Washington. 
HNF-6273

Rev. 0 
HNF-6273

Rev. 0

APPENDIX A

BASIS FOR IXM RELEASE 
HNF-6273

Rev. 0 
HNF-6273

Rev. 0

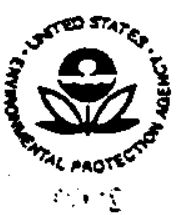

Phil Loscoe, Director Spent Nuclear Fuels Project Office U.S. Department of Energy P.O. Box 550, S7-41 Richland, WA 99352

\section{UNITED STATES ENVIRONMENTAL PROTECTION AGENCY}

REGION TOHANFORDPROJECT OFFICE

712 Switt Boulevard, Sulte 5

Richland, Washington 99352

April 11, 2000

Owen Robertson, Senior Program Manager

Environmental Restoration/Waste Disposal

U.S. Department of Energy

P.O. Box 550, HO-12

Richland, WA 99352

SUBJECT: Treatment of Potentially LDR Waste in LXMS From K Basins Prior to Disposal at ERDE.

Dear Messrs. Loscoe and Robertson:

During the Data Quality Objectives (DQO) process for debris fom the $\mathrm{K}$ Basins, BHI personnel identified disposal of ion exchange modules (DXMs) in ERDF as an issue. Since the $K$ Basins project has become a CERCLA remedial action, DXMs are to be disposed in ERDF. The LXMs contain resins used to remove radionuclides from the $\mathrm{K}$ Basins water, and in the process have the potential to accumulate sufficient RCRA-regulated metals to become land disposal restricted (LDR) waste. The resin chambers are fabricated as an integral component of the IXMs and are not accessable for sampling or treatment. Historically, as the DXMs have been taken out of service, they have been drained and the plumbing orifices filted with HEPA filters prior to disposal in the low level burial grounds.

In response to the issue raised by BHI staff on March 9,2000 during a DQO global issues resolution meeting, I provided the following strategy to resolve this issue:

EPA will consider the IXMIs - drained and fitted with HEPA filters - as having met the macroencapsulation immobilization technology as an alternative treatment standard for hazardous debris under 40 CFR 268.45. Therefore:

The DXMs do not need to be sampled prior to disposal at ERDF.

The bounding waste profile (including designation as non-TRU waste) for the IXMs may be based on operational records of volume of water treated and influent concentrations of metals and radionuclides.

This letter reaffirms EPA's position on macroencapsulation of [XMs. If you have any questions, please call me at (509) 376-9884.

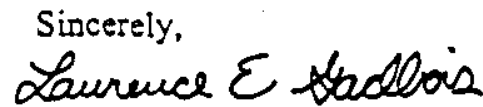

Laureace E. Gadbois K Basins Project Manager 
HNF-6273

Rev. 0

Messrs. Loscoe and Robertson

-2 -

April 11, 2000

cc: Julie Atwood, BHI

Paul Day, MACTEC

Oscar Holgado, DOE

Randy Jackson, BHI

Chris Lucas, FH

Mitzi Miller, EQM

Jeff Westcott, WMH

Administrative Record, 100-KR-2 


\section{Environmenta! \\ Restoration Contractor \\ Interoffice Memorandum}

TO:

COPiEs:
A. R. Michael $\mathrm{H} 0-17$

B. P. Moyers $T 2.05$

B. D. Schilperoor T2-05

J. M. Atwood HO-21

R. L. Weiss H9-03

J. D. Arana L5-64

Document and Info Services HO-O9
RECEIVED APR 052000 BY DIS

DATR:

rROM:

April 4, 2000
Job No. 22192

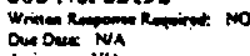
raions NA Clome CCN: NA Ti: $\sin ^{2}$ TSO $\mathrm{NA}_{\mathrm{A}}$

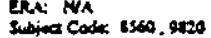

\section{A. Casbon houc} ERDF $T 2-05 / 373-7328$

Ref: Memo C. D. Lucas to File, Hydrogen Concentrations in Spent LXM's, Westinghouse Hanford Company, OAWH-074-95

\section{SUAECT: K-BASINS IXM GAS GENERATION}

One of the waste streams that the Spent Nuclear Fuels (SNF) project would like to send to the Environmental Restoration Disposal Facility (ERDF) consists of a number of Ion Exchange Modules (DXMs). The IXMs generate gasses through radiolytic interactions with the water and resin remaining within them. My analysis indicates that the total amount of gas generated will have no deleterious effect on the operations or closure of the ERDF. My conclusion is based on the relatively small amounts of gas generated relative to available pore space within the waste soils disposed in the ERDF.

The ERDF will receive one EXM per month for the duration of the SNF project. This will yield a total of 24 to 36 IXMs. After reviewing the document referenced above, Richard Weiss of CHI determined that each IXM would generate between 30 and 150 liters per year of gasses. The gasses will contain hydrogen in concentrations ranging from $<1 \%$ to $75 \%$ of the total gas volume. The gas generation rate and total gas generated is tied to the half-life of the primary radionuclides Cs- 137 and Sr-90 (approximately 30 years). A calculation by Joel Arana, Environmental Technical Lead, shows that an IXM generating 150 liters per year initially; generating a total of 6,294 liters of hydrogen in the space of 7 half-lives. I confirmed Joel's calculation using a different methodology for my calculation.

The total gas generated will be 6,294 liters $\times 36$ IXMs $=226,584$ liters. This equals $8,002 \mathrm{ft}^{3}$ or 296 $\mathrm{yd}^{3}$. Assuming that a total of $6,000,000 \mathrm{yd}^{3}$ of waste will ultimately be placed in the ERDF the gas will account for only $4.9 \mathrm{E}-5$ or $0.005 \%$ of the total volume. This is far less than the available pore space in the ERDF soils, no matter how highly compacted. Therefore, the gasses generated will not cause any problems with subsidence in the landfill.

MAC:mac

Attachments: e-mail, R. L. Weiss to M. A. Casbon, "DXM Gas Generation," dated April 4, 2000 e-mail; J. D. Arana to M. A. Casbon, "Hydrogen Generation," dated March 31, 2000 
HNF-6273

Rev. 0

077459

Casbon, Michael A (Mike)

$\begin{array}{ll}\text { From: } & \text { Weiss, Richard L } \\ \text { Sent: } & \text { Tuesday, April } 04,2000 \text { 7:44 AM } \\ \text { To: } & \text { Casbon, Michael A (Mike) } \\ \text { Subject: } & \text { RE: IXM Gas Generation }\end{array}$

Mike,

Sorry for the delay on this.

1 reviewed the gas generation portions of the documents that you sent me. I did not attempt to recreate the calculations and no obvious flaws were noted. The range of generation rate and composition was bracketed due to many variables in the calculations. Based on the information presented gas generation rates will be approximately 30-150 liters per year.

The hydrogen concentration potentially ranges from very low $<1 \%$ to as great as $75 \%$. This generation should continue for the foreseeable tuture and the generation rate will "decay" at the same rate the radioactivity present decays (approximately 30 y half-life from the primary radionuclides Cs-137 \& SR-90).

Let me know if you have any additional questions.

Rich

\begin{tabular}{|c|c|}
\hline $\begin{array}{l}\text { Original Me } \\
\text { From: } \\
\text { Sent } \\
\text { To: } \\
\text { Cc: } \\
\text { Subject: }\end{array}$ & $\begin{array}{l}\text { 9o- } \\
\text { Caston, Mlchad A (Mike) } \\
\text { Friday, March } 31,2000 \text { 9:52 AM } \\
\text { Woiss, Richard L } \\
\text { Atwood. Julie M } \\
\text { LM Gas Generation }\end{array}$ \\
\hline
\end{tabular}

Rich,

Thank you for the information on K-Basin lon Exchange Module gas generation. Please send me an $\theta-$ mail to back up our conversation. I will use a 30 yr "half life" of the first year's maximum gas generation rate to calculate a total ( 7 half ives) gas volume to be generated.

MAC 
HNF-6273

Rev. 0

077459

Casbon, Michael A (Mike)

\section{From: \\ Sent: \\ To: \\ Subject:}

Importance:
Arana, Joel D

Friday, March 31. 2000 10:08 AM

Casbon, Michael A (Mike)

Hydrogen Generation

High

Mike,

Good thing you had me look at that formula. Your number was high by a factor of about 38 ! I did infact have to integrate. The actual number came out to be about 6,294 total liters produced over the period of 7 half-lives. 1 have attached the formula as a Word document

Call me if you have any questions.

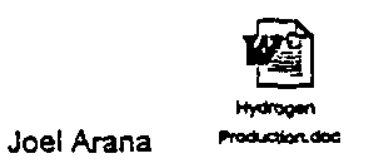


HNF-6273

Rev. 0

077459

Using the simple decay equation: $A_{r}=A_{0} e^{-\lambda s}$ where $A_{0}$ is your conservative hydrogen generation estimate of 150 liters/year, $t$ is the time of about 7 half-lives (210 years) and $\lambda$ is your decay constant; $\lambda=\ln 2 / 30 \mathrm{yr}$ or $2.31 \mathrm{E}-2 \mathrm{yr}^{-1}$.

Since we want to know the cumulative hydrogen that has been generated over the 210 year period we must integrate. $\int A_{0} e^{-\lambda s} d t$ is simply $A_{0}\left(e^{-\lambda /} / \lambda\right)$. Evaluating this from 0 to 210 years yields a total of 6.294 liters of hydrogen gas produced in 210 years or 7 half. lives.

Looking at a longer time frame is not necessary. After 210 years less than a liter a year of hydrogen is produced. 


\section{CCN \# 077459}

\section{CHRONED BY: MKW DATE: $4 / 4 / 00$}

MACRO COMPLETED: YES $\leftrightharpoons$

REPRO/DIST. COMPLETED: YES

No

DISTRIBUTED BY: $M<\omega$

CANCELLED BY: $n / \mathbb{A}$.

OU: ERDF

Distributed To:

$\begin{array}{ll}\square & \text { E. L. Adamson (BHI), T2-05 } \\ \square & \text { J.M. Atwood (BHD), T2-05 } \\ \square & \text { R. H. Bidstrup (BHI), T2-05 } \\ \square & \text { M. A. Casbon (BHI), T2-05 } \\ \square & \text { V. R. Dronen (BHI), T2-05 } \\ \square & \text { F. O. Lamb (BHI), T2-05 }\end{array}$

DOCSOPEN 121203,04,21,97

\author{
E. D. Marvin (BHI), H0-04 \\ $\square$ B. P. Moyers (BHI), T2-05 \\ D. A. Riley (BHI), T2.05 \\ B B. D. Schilperoort (BH), T2-05 \\ S. G. Thieme (BHI), X0-17 \\ C D. R. Thomas (BHI), T2-04
}

TO BE COMPLETED BY DIS

DATA ENTRY BY: DNWL SCANNED BY: me m/5 RECORD TYPE: IDM 
HNF-6273

Rev. 0

A-8 
HNF-6273

Rev. 0

\section{APPENDIX B}

\section{RADIONUCLIDE/ISOTOPES COPC SELECTION}


HNF-6273

Rev. 0 
HNF-6273

Rev. 0

To: Mitzi Miller

From: Al Robinson

Date: $2 / 28 / 00$

Subject: Contaminants of Potential Concern (COPCs) and Rationale for Selection

The source term for all of the radionuclides that could reasonably be expected in the KBasin is from N-Reactor fuel and associated activation products. The selection of COPCs was conducted by first listing all of the radionuclides that have been reported as present in the fuel or measured during historical characterization of the KE, KW, N, or 105-C fuel storage basins. Several of the documents refer to computerized simulations of the radionuclides content of nuclear fuel using the ORIGEN II computer code. The five sources that were used to supply this data are:

1. BHI Archive Number 0161426. "105-N Basin Deactivation Project Radioactive Waste Management Archive, Volumes 1 and 2". July, 1998. (BHI 1998).

2. WHC-SD-TP-SEP-028, Rev 0. "Safety Evaluation for Packaging the N Reactor/Single Pass Reactor Fuel Characterization Shipments", Oct. 1994. (WHC 1994)

3. WHC-SD-NR-RPT-005, Rev 0. "Characterization of Radioactive Waste at 100 Area", Nov. 1990. Written by John DeVanney (DeVanney 1990)

4. WHC-EP-0063-4 (Page Change 5), "Hanford Solid Waste Acceptance Criteria", April 1996, Appendix K, Table K-1, (WHC 1996)

5. Letter to CA Palmquist from S.P Roblyer Fluor Daniel Northwest, Inc. dated May 14, 1997. Subject: Radionuclides in the 105-C Fuel Transfer Pit Sediments.

The initial list of COPCs (Table 1) contained 80 radionuclides, which had been discussed as estimated components of the $\mathrm{N}$-reactor fuel from fission or activation processes or had been reported in work associated with the fuel or the basin. The DeVanney report (DeVanney 1990) contained extensive analyses of sample from the $\mathrm{KE}$ and $\mathrm{KW}$ basin areas. These data provided valuable estimates of several radionuclides that had not been estimated from other sources (e.g. Ni-59, Cr-51, Mn-54) and the estimated ratios that were also included in the initial list. Since the data was obtained or calculated at various times over the last 16 years, the next step was to decay correct the estimated amount of each radionuclide. All decay corrections were performed to the year 2000. In order to put all of the radionuclides from the various sources on a normalized basis, all final estimates of radionuclide content of the fuel (from ORIGEN runs) or samples from KW and KE basins, were converted to a percent 
of the estimated Cs-137 concentration. For instance if the reference indicated that the fuel would contain $500 \mathrm{Ci}$ of $\mathrm{Sr}-90$ and $1000 \mathrm{Ci}$ of $\mathrm{Cs}-137$, the percentage entered into Table 1 would be $50 \%$.

Also tabulated in Table 1 are the ERDF radiological waste acceptance criteria BHI 1998). The acceptance criteria were also converted to a percentage of Cs-137 in order to assist the final selection of COPCs. The percentage of Cs-137 in the last column to the right in Table 1 represents the allowable maximum percentage of each radionuclide compared to Cs-137 at the maximum allowable concentration $\left(\mathrm{Ci}^{2} / \mathrm{m}^{3}\right)$. During the selection process this column was evaluated against each criteria used to eliminate radionuclides from the COPC list. If any of the criteria would eliminate a radionuclide that had an estimated Cs-137 ratio within a factor of 10 of the maximum allowable contamination limits at ERDF, then the radionuclide was retained. The process of potential COPC elimination proceeded as follows:

1. The first step of the process involved eliminating any of the radionuclides that were not part of the N-Reactor uranium fuel cycle process. These radionuclides included radium, and thorium.

2. The next step involved the elimination of any radionuclides that were gaseous or have a radioactive half-life of less than 1 year.

3. Also eliminated were any beta/gamma emitting radionuclides that were estimated to be present at less than $1 \%$ of the Cs-137 activity of the waste (in the year 2000) by all of the five references listed.

4. For alpha emitters and transuranic radionuclides, any radionuclides that were less than $0.1 \%$ of the $\mathrm{Cs}-137$ activity were eliminated.

These four step process was chosen to adequately define the term "major radionuclide content" in the ERDF WAC (BHI-00139). Table 2, lists only the radionuclides that are left as COPCs after application of the elimination process. Two isotopes of Europium (Eu-152, Eu-155) were left in Table 2 even though they did not meet the criterion $(>1 \%$ of Cs-137) for inclusion. They were left in because they have been found in other on site graphite reactors and will be reported along with Eu-154, which was in the proposed final list of COPCs. Similarly, Ni-63 (an activation product) has been found in other graphite reactors at concentrations approximately equal to $\mathrm{Sr}-90$ and $\mathrm{Cs}-137$, thus has remained a COPC even though it is estimated by the ORIGEN run to be present in the fuel at less than $1 \%$ of the Cs-137. Also listed in Table 2 are the estimated ratios of the activity of each radionuclide compared to $\mathrm{Cs}-137$ from historical data that may be used to estimate hard-to-measure radionuclides (e.g. Sm-151, H-3). The KE and KW basins have been listed separately because of the significant differences that were found in historical analyses of samples that are applicable to WSs from the two facilities (DeVanney 1996). The ratios listed in Table 2 may be used to estimate the radioactive content of the hard to measure COPCs (e.g. pure beta emitters, alpha emitters) in the waste from analysis of a subset of the easy to measure COPCs (e.g. gamma emitters such as $\mathrm{Cs} / \mathrm{Ba}-137$ ). In the event that the ratios in Table 2 are used, the most conservative applicable ratio will be used. In most cases there is more recent data for selected radionuclides in each specific WS. The more recent WS specific data will be 
Rev. 0

used where available to obtain the most accurate estimate of the radionuclide content of the waste. The ratios used to estimate the radionuclide content of the waste and/or to estimate the hard to measure radionuclides, will be referenced in the waste shipment documentation. It should also be noted that in Table 1 and 2 the measured ratios of various isotopes to Cs-137 in the DeVanney report vary markedly. Recent NDA for WS profiles common to KE and $\mathrm{KW}$ indicate similar concentrations of the major radionuclides (e.g. Pu-239, Sr-90, Cs-137) in the waste. 


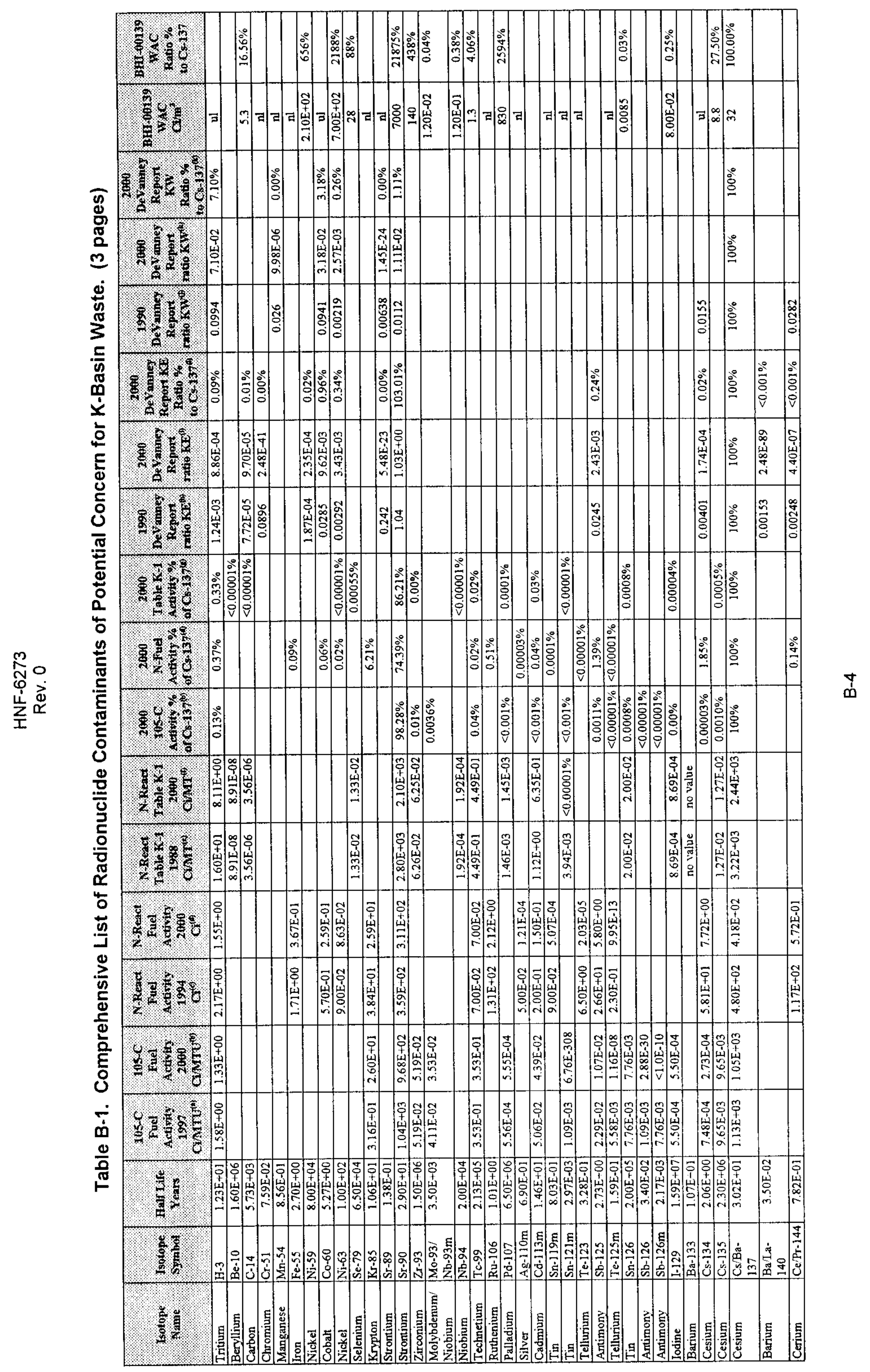




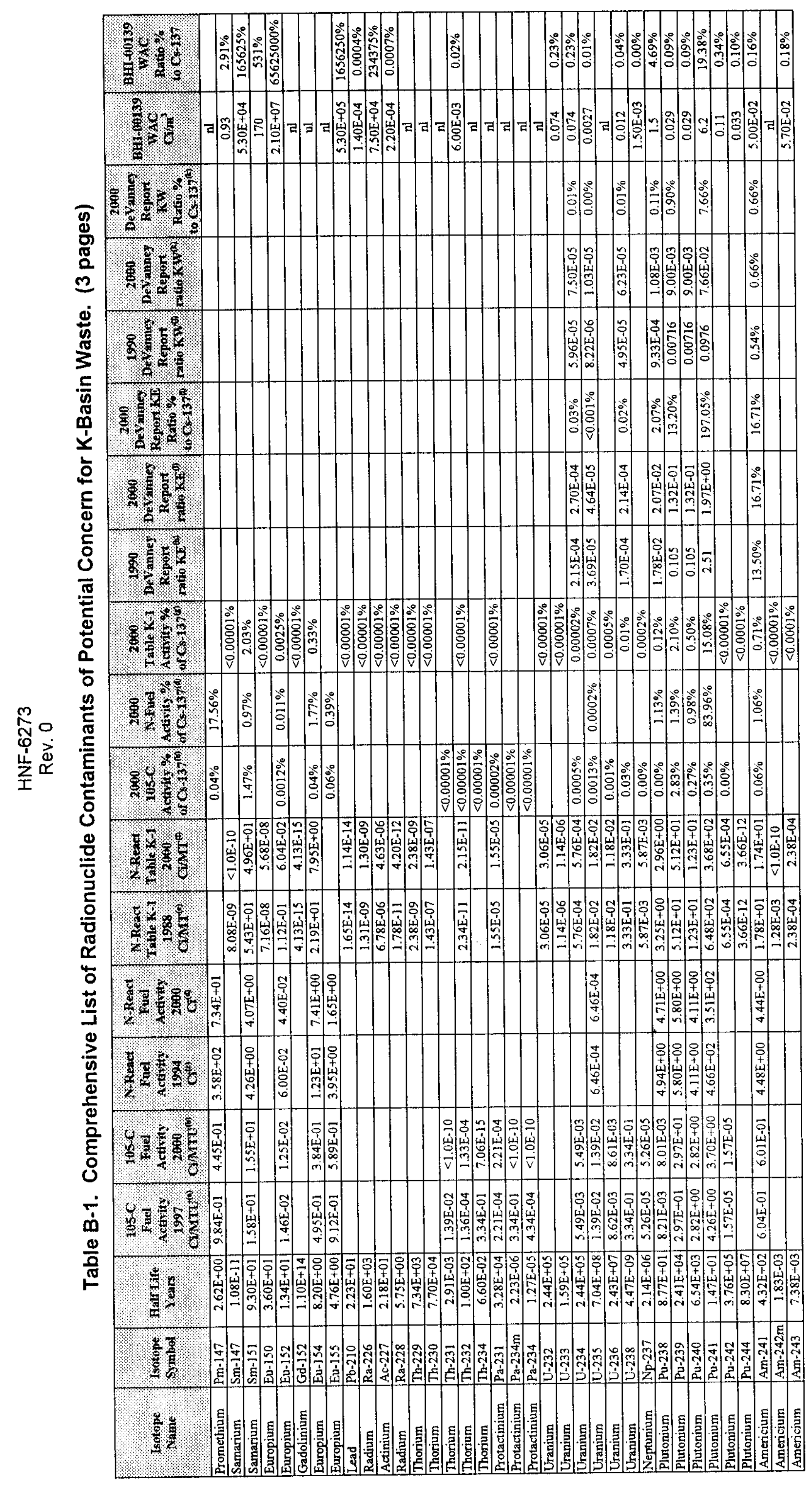

๓ீ 


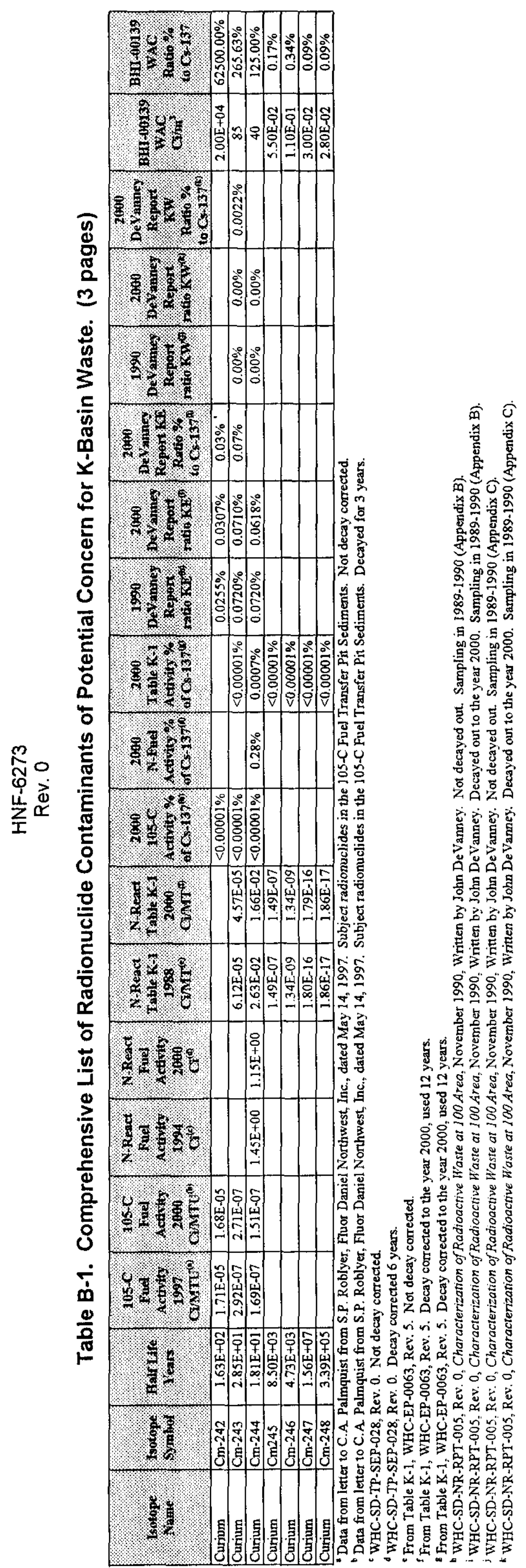

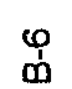


HNF-6273

Rev. 0

Table B-2. Final COPCs for K-Basin Waste.

\begin{tabular}{|c|c|c|c|c|c|c|c|c|}
\hline $\begin{array}{l}\text { Isotope } \\
\text { Name }\end{array}$ & $\begin{array}{l}\text { Isotope } \\
\text { symbol }\end{array}$ & $\begin{array}{l}\text { Half Life } \\
\text { Years }\end{array}$ & $\begin{array}{l}2000 \\
105 . c \\
\text { Activity \% } \\
\text { of Cs-137. }\end{array}$ & $\begin{array}{l}2000 \\
\text { Arfuel } \\
\text { Activity } \\
\% \text { cs } 137\end{array}$ & $\begin{array}{l}2000 \\
\text { Table K-1 } \\
\text { Activity } \% \\
\text { of Cs-137 }\end{array}$ & $\begin{array}{l}2000 \\
\text { Devanney } \\
\text { Report kE } \\
\text { Ratio } \% \\
\text { to Cs-137. }\end{array}$ & $\begin{array}{l}2000 \\
\text { DeVanney } \\
\text { Report kW } \\
\text { Ratio } \% \\
\text { to Cs } 137\end{array}$ & $\begin{array}{l}\text { BHI } \\
00139 \\
\text { WAC } \\
\text { Ratio \% } \\
\text { to Cs } 137\end{array}$ \\
\hline Tritium & $\mathrm{H}-3$ & $1.23 \mathrm{E}+01$ & $0.13 \%$ & $0.37 \%$ & $0.33 \%$ & $0.09 \%$ & $7.10 \%$ & \\
\hline Cobalt & Co-60 & $5.27 \mathrm{E}+00$ & & $0.06 \%$ & & $0.96 \%$ & $3.18 \%$ & \\
\hline Nickel & $\mathrm{Ni}-63$ & $1.00 \mathrm{E}+02$ & & $0.02 \%$ & $<0.00001 \%$ & $0.34 \%$ & $0.26 \%$ & $2188 \%$ \\
\hline Strontium & Sr-90 & $2.90 E+01$ & $98.28 \%$ & $74.39 \%$ & $86.21 \%$ & $103.01 \%$ & $1.11 \%$ & $21875 \%$ \\
\hline Antimony & $S b-125$ & $2.73 E+00$ & $0.0011 \%$ & $1.39 \%$ & & $0.24 \%$ & & \\
\hline Cesium & $\begin{array}{l}\text { Cs/Ba- } \\
137\end{array}$ & $3.02 E+01$ & $100 \%$ & $100 \%$ & $100 \%$ & $100 \%$ & $100 \%$ & $100.00 \%$ \\
\hline $\begin{array}{l}\text { Promethiu } \\
\text { m }\end{array}$ & $\mathrm{Pm}-147$ & $2.62 \mathrm{E}+00$ & $\%$ & $17.56 \%$ & & & & \\
\hline Samarium & $5 m-151$ & $9.30 E+01$ & $1.47 \%$ & $0.97 \%$ & $2.03 \%$ & & & $165625 \%$ \\
\hline Europium & Eu-152 & $1.34 \mathrm{E}+01$ & $0.0012 \%$ & $0.011 \%$ & $0.0025 \%$ & & & \begin{tabular}{|c}
65625000 \\
$\%$ \\
\end{tabular} \\
\hline Europium & Eu-154 & $8.20 E+00$ & $0.04 \%$ & $1 . \overline{77 \%}$ & $0.33 \%$ & & & \\
\hline Europium & Eu-155 & $4.76 \mathrm{E}+00$ & $0.06 \%$ & $0.39 \%$ & & & & \\
\hline Uranium & U-235 & $7.04 E+08$ & $0.0013 \%$ & $0.0002 \%$ & $0.0007 \%$ & $0.0046 \%$ & $0.0010 \%$ & $0.01 \%$ \\
\hline Uranium & $U-238$ & $4.47 \mathrm{E}+09$ & $0.03 \%$ & & $0.01 \%$ & $0.02 \%$ & $0.01 \%$ & $0.04 \%$ \\
\hline Plutonium & Pu-238 & $8.77 \mathrm{E}+01$ & $0.00 \%$ & $1.13 \%$ & $0.12 \%$ & $2.07 \%$ & $0.11 \%$ & $4.69 \%$ \\
\hline Plutonium & $\mathrm{Pu}-239$ & $2.41 \mathrm{E}+04$ & $2.83 \%$ & $1.39 \%$ & $2.10 \%$ & $13.20 \%$ & $0.90 \%$ & $0.09 \%$ \\
\hline Plutonium & $P u-240$ & $6.54 E+03$ & $0.27 \%$ & $0.98 \%$ & $0.50 \%$ & & & $0.09 \%$ \\
\hline Plutonium & $\mathrm{Pu}-241$ & $1.47 \mathrm{E}+01$ & $0.35 \%$ & $83.96 \%$ & $15.08 \%$ & $197.05 \%$ & $7.66 \%$ & $19.38 \%$ \\
\hline Americium & $A m-241$ & $4.32 \mathrm{E}+02$ & $0.06 \%$ & $1.06 \%$ & $0.71 \%$ & $16.71 \%$ & $0.66 \%$ & $0.16 \%$ \\
\hline Curium & $\mathrm{Cm}-244$ & $1.81 E+01$ & $0.00001 \%$ & $0.28 \%$ & $0.0007 \%$ & & & $0.09 \%$ \\
\hline
\end{tabular}


HNF-6273

Rev. 0 
HNF-6273

Rev. 0

APPENDIX C

ESTIMATION OF METALS AND PCBS SORBED ONTO ION-EXCHANGE MODULES AT K BASINS

C-i 
HNF-6273

Rev. 0

C-ii 
Rev. 0

\section{Estimation of Metals and PCBs Sorbed onto lon-exchange Modules at K-Basins}

\author{
Spreadsheet Prepared by: \\ Spreadsheet Prepared on: \\ concentration
}

\author{
CW Miller, EQM \\ 9-Mar-00 *note corrected values for TC screening
}

The following spreadsheet was used to conservatively estimate the potential content of PCBs and toxic metals that may sorb onto the ion exchange resins contained within the ion exchange modules used to maintain water quality in the K-Basins. To support these calculations, the following factors were considered:

1. The contaminants of concern were assumed to be present in the basin water at the reported detection limit(s). The samples used for this evaluation were reported to have been collected from the middle of the basin.

2. The decontamination factor for the IX resin was assumed to be $100 \%$ (i.e., all dissolved constituents passing through the resin are sorbed).

3. The metals are assumed to be sorbed by the anion/cation exchange complex of the resin.

4. The PCBs are assumed to be sorbed preferentially to the non-polar ion exchange resin matrix.

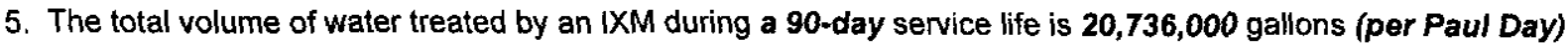

6. The weight of an IXM, including the concrete housing is 42,000 pounds (per Rodney Jochen)

7. The volume of $\mathrm{IX}$ resins and void space within the exchange columns is 21 cubic feet (per Rodney Jochen)

8. The density of hydrated ion exchange resin is assumed to be $1.0 \mathrm{~g} / \mathrm{ml}$.

Estimated PCB Loading of IXM

\begin{tabular}{|c|c|c|c|c|c|c|}
\hline \multicolumn{2}{|c|}{ Water Volume } & \multirow{2}{*}{$\frac{\text { PCB Conc. }}{(\mathrm{mg} / \mathrm{l})}$} & \multirow{2}{*}{$\frac{\text { PCB Mass }}{(\mathrm{mg})}$} & \multicolumn{2}{|c|}{ IXM Mass } & \\
\hline (gallons) & (liters) & & & $(\mathrm{lb})$ & $(\mathrm{kg})$ & (mg/kg) \\
\hline 20736000 & 78485760 & $<0.50$ & 39242880 & 42,000 & 19,051 & 2,060 \\
\hline
\end{tabular}

Exceeds

Screening Level?

Yes

Estimated Lead Loading of IXM (selenium and arsenic have same detection limit)

\begin{tabular}{|c|c|c|c|c|c|c|}
\hline \multicolumn{2}{|c|}{ Water Volume } & \multirow{2}{*}{$\frac{\text { Lead Conc. }}{(\mathrm{mg} / \mathrm{l})}$} & \multirow{2}{*}{$\frac{\text { Lead Mass }}{\text { (mg) }}$} & \multicolumn{2}{|c|}{ IXM Mass } & 6 \\
\hline (gallons) & (liters) & & & (lb) & $(\mathrm{kg})$ & $(\mathrm{mg} / \mathrm{kg})$ \\
\hline 20736000 & 78485760 & $<0.10$ & 7848576 & 42,000 & $\overline{19,051}$ & 412 \\
\hline
\end{tabular}

Estimated Chromium Loading of IXM (silver and barium have same detection limit)

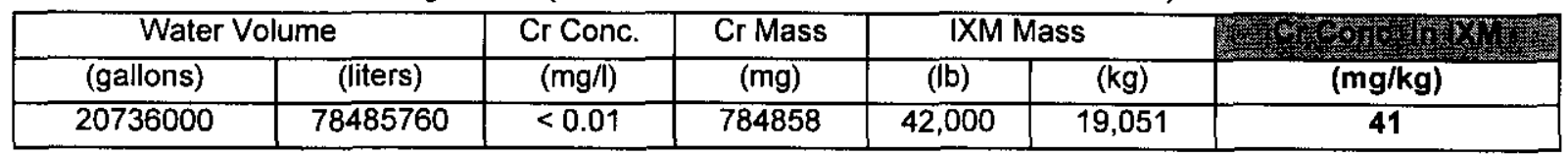

No

Estimated Cadmium Loading of IXM

\begin{tabular}{|c|c|c|c|c|c|c|}
\hline \multicolumn{2}{|c|}{ Water Volume } & \multirow{2}{*}{$\frac{\text { Cd Conc. }}{(\mathrm{mg} / \mathrm{l})}$} & \multirow{2}{*}{$\frac{\text { Cd Mass }}{(\mathrm{mg})}$} & \multicolumn{2}{|c|}{ IXM Mass } & \\
\hline (gallons) & (liters) & & & (Ib) & $(\mathrm{kg})$ & $(\mathrm{mg} / \mathrm{kg})$ \\
\hline 20736000 & 78485760 & $<0.001$ & 78486 & 42,000 & 19,051 & 4.12 \\
\hline
\end{tabular}

No

Toxicity Characteristic Screening Levels for Total Metal Analysis (TCLP x 20)

\begin{tabular}{|c|c|c|c|c|c|c|c|}
\hline $\begin{array}{c}\text { Arsenic } \\
(\mathrm{mg} / \mathrm{kg})\end{array}$ & $\begin{array}{c}\text { Barium } \\
(\mathrm{mg} / \mathrm{kg})\end{array}$ & $\begin{array}{c}\text { Cadmium } \\
(\mathrm{mg} / \mathrm{kg})\end{array}$ & $\begin{array}{c}\text { Chromium } \\
(\mathrm{mg} / \mathrm{kg})\end{array}$ & $\begin{array}{c}\text { Lead } \\
(\mathrm{mg} / \mathrm{kg})\end{array}$ & $\begin{array}{c}\text { Mercury } \\
(\mathrm{mg} / \mathrm{kg})\end{array}$ & $\begin{array}{c}\text { Selenium } \\
(\mathrm{mg} / \mathrm{kg})\end{array}$ & $\begin{array}{c}\text { Silver } \\
(\mathrm{mg} / \mathrm{kg})\end{array}$ \\
100 & 2000 & 20 & 100 & 100 & 4 & 20 & 100 \\
\hline
\end{tabular}


HNF-6273

Rev. 0

Maximum Concentration of Toxic Metals Reported in KE-Basin Sludge

\begin{tabular}{|c|c|c|c|c|c|c|c|}
\hline $\begin{array}{c}\text { Arsenic } \\
(\mathrm{mg} / \mathrm{kg}) \\
\text { not reported }\end{array}$ & $\begin{array}{c}\text { Barium } \\
\text { (mg/kg) } \\
430\end{array}$ & $\begin{array}{c}\text { Cadmium } \\
(\mathrm{mg} / \mathrm{kg}) \\
37\end{array}$ & $\begin{array}{c}\text { Chromium } \\
(\mathrm{mg} / \mathrm{kg}) \\
957\end{array}$ & $\begin{array}{c}\text { Lead } \\
(\mathrm{mg} / \mathrm{kg}) \\
480\end{array}$ & $\begin{array}{c}\text { Mercury } \\
\text { (mg/kg) } \\
\text { not reported }\end{array}$ & $\begin{array}{c}\text { Selenium } \\
(\mathrm{mg} / \mathrm{kg}) \\
133\end{array}$ & $\begin{array}{c}\text { Silver } \\
(\mathrm{mg} / \mathrm{kg}) \\
13\end{array}$ \\
\hline
\end{tabular}

PCBs Measured in KE-Basin Sludge Samples (per PCB Spill Response Plan)

\begin{tabular}{|lc|}
\hline Samples Collected & 21 \\
\hline $\begin{array}{l}\text { Samples Screened for } \\
\text { PCBs }\end{array}$ & 10 \\
\hline $\begin{array}{l}\text { Samples with PCBs } \\
\text { detected }\end{array}$ & 3 \\
\hline $\begin{array}{l}\text { Maximum PCBs } \\
\text { detected }\end{array}$ & $220 \mathrm{mg} / \mathrm{kg}$ \\
\hline Minimum PCBs detected & $47 \mathrm{mg} / \mathrm{kg}$ \\
\hline
\end{tabular}

The following COPC concentrations were reported in a sample of IXM outlet water in August 1997.

These values are not comparable to the non-detects reported for the mid-basin samples and

cannot be used to support IXM loading

calculations.

\begin{tabular}{|c|}
\hline $\begin{array}{c}\text { PCBs } \\
\text { (ug/l) }\end{array}$ \\
\hline$<1.0$ \\
\hline
\end{tabular}

\begin{tabular}{|c|c|c|c|c|c|c|c|}
\hline $\begin{array}{c}\text { Arsenic } \\
(\mathrm{ug} / \mathrm{l})\end{array}$ & $\begin{array}{c}\text { Barium } \\
(\mathrm{ug} / \mathrm{l})\end{array}$ & $\begin{array}{c}\text { Cadmium } \\
(\mathrm{ug} / \mathrm{l})\end{array}$ & $\begin{array}{c}\text { Chromium } \\
(\mathrm{ug} / \mathrm{l})\end{array}$ & $\begin{array}{c}\text { Lead } \\
(\mathrm{ug} / \mathrm{l})\end{array}$ & $\begin{array}{c}\text { Mercury } \\
(\mathrm{ug} / \mathrm{l})\end{array}$ & $\begin{array}{c}\text { Selenium } \\
(\mathrm{ug} / \mathrm{l})\end{array}$ & $\begin{array}{c}\text { Silver } \\
(\mathrm{ug} / \mathrm{l})\end{array}$ \\
\hline$<2.3$ & 5.8 & $<3.4$ & $<2.7$ & $<1.5$ & $<0.10$ & $<3.6$ & $<3.6$ \\
\hline
\end{tabular}


HNF-6273

Rev. 0

\section{APPENDIX D \\ DRAFT WASTE PROFILE CONTAINING DATA COLLECTED AFTER SPRAY WASHING \\ TO REMOVE PCB/TCMETALS}

Only Appendices B \& F from

Profile Apply and are included.

D-i 
HNF-6273

Rev. 0

D-ii 
HNF-6273

Rev. 0

D.1 RADIATION EXPOSURE EVALUATION FOR DETERMINATION OF WORKER RADIOLOGICAL CONTROLS

D-1 
HNF-6273

Rev. 0

Attachment $C$ Calculations

QUESTION: How arch = luelge would regulate the wast is as a Mixed waste?

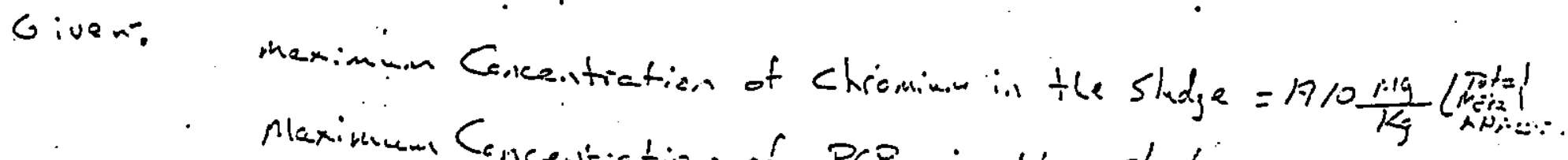

Maximum Cenceritiation of $P C E=$ in the sluolge $=200 \frac{\mathrm{ma}}{\mathrm{kg}}$

Peguktory limit for Chrcmion... $=5 \frac{\mathrm{mg}}{L}$. (TemP Extras)

PCB Characteristic of Persistence $=100 \frac{\mathrm{diq}}{\mathrm{K}}$

Equation: $\quad \frac{C_{3} X_{3}}{X_{W}}=R_{L}$

Where: $C_{S}=$ Coricenticution of Sludge for chromium oR PCO

$x_{s}=$ mass of sludge

$X_{u}=$ mass of Waste.

$P_{L}=$ Regulatory limit Concentration for Chrewiolen oo PCB

solution: $X_{10}^{\prime}=X_{s}+X_{m} \quad$ where: $x_{\text {in }}$ is weight of natal waste

for Chroininin: Dor Code

$$
\begin{aligned}
& 1910 \frac{m: 1}{x_{j}}\left(X_{s}\right) \rightarrow \text { Assuming } 20 \text { Til dilation fir } \\
& \frac{1910 \frac{m}{r_{y}}\left(x_{s}\right)}{x_{s}+x_{m}}=5 \frac{1 \% s}{L} \times \frac{20 L}{k_{j}} \text { Density is } 1 \% / m L . \\
& \Rightarrow 100 x_{3}+100 x_{\mathrm{m}}=1910 x_{3} . \\
& \Rightarrow \quad x_{m}=18.1 x_{5}
\end{aligned}
$$

D-2 
HNF-6273

Rev. 0

Calculations Centinued:

$$
\begin{aligned}
& \text { ma.ss of inetal }=11 \mathrm{~b} \times \frac{\mathrm{kg}_{\mathrm{g}}}{2.2 \mathrm{~b}}=\cos 1 / 54 \mathrm{~kg} \\
& \Rightarrow 0.45 \% 1 k_{y}=18.1 x_{3} \\
& \Rightarrow x_{3}=0.025 k_{j} \times 2.2 \frac{1 b}{k_{g}}=0.055 \mathrm{ll} \text { regulate for Con }
\end{aligned}
$$

for PCPs: Persistance

$$
\begin{aligned}
& \frac{220 \frac{\mathrm{ng}}{k_{\mathrm{g}}}\left(x_{s}\right)}{x_{\mathrm{g}} \div x_{\mathrm{m}}^{\prime}}=100 \frac{\mathrm{mg}}{\mathrm{kg}} \\
& \Rightarrow 100 x_{s} \ddot{100 x_{1}}=220 x_{s} \\
& \Rightarrow \quad \therefore, \therefore=i=x_{s} \\
& x_{3}=.378 \mathrm{~kg} \times \frac{2.216}{1 \mathrm{~g}}=0.83 \mathrm{lb}=\text { regulats for } P C B_{s}
\end{aligned}
$$$$
6-26-9697
$$$$
\text { zisth for. }
$$

D-3 
HNF-6273

Rev. 0 
HNF-6273

Rev. 0

APPENDIXE

LEAD INVENTORY

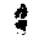


HNF-6273

Rev. 0

E-ii 


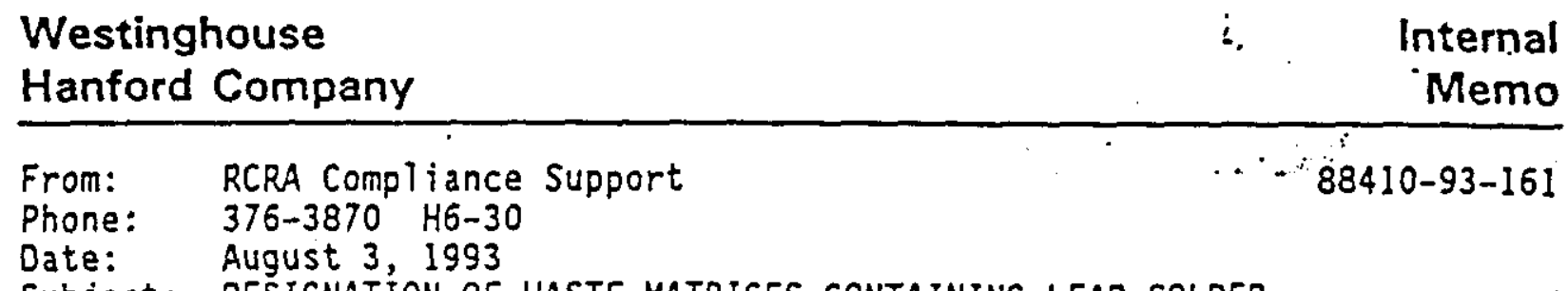

Subject: DESIGNATION OF WASTE MATRICES CONTAINING LEAD SOLDER

To:

\begin{tabular}{|c|c|}
\hline P. Will is & T3-05 \\
\hline $\begin{array}{l}\text { cc: D. L. Allen } \\
\text { R. L. Austin } \\
\text { B. J. Dixon } \\
\text { B. G. Erlandson } \\
\text { O. T. Foley } \\
\text { J. E. Gamin } \\
\text { E. M. Greager } \\
\text { P. J. Mackey } \\
\text { A. G. Miskho } \\
\text { B. L. Vedder } \\
\text { H. T. Tilden } \\
\text { W. E. Toebe } \\
\text { G. C. Triner } \\
\text { RCS Staff }(10) \\
\text { MJS File/LB }\end{array}$ & $\begin{array}{l}T 3-05 \\
T 3-04 \\
67-33 \\
H 6-20 \\
L 8-09 \\
G 6-57 \\
H 6-30 A M G \\
B 3-15 \\
H 6-30 \pm C \\
H 6-22 \\
P 7-68 \\
\left.H 6-22, L C^{\prime}\right] \\
N 3-13\end{array}$ \\
\hline
\end{tabular}

Per this memo, a dangerous waste designation (OW-D008) is being established for certain waste matrices containing lead solder. In addition, this letter is providing a methodology to complete waste designations on waste matrices which contain lead solder. This letter is not encouraging the dismantling of equipment to determine lead solder percentages or to remove lead solder components from equipment prior to excessing or disposal.

When applicable, best engineering judgement shall be used for determining the lead solder weight percentages in a waste matrix. In most cases, this judgement will constitute sufficient process knowledge to determine that equipment including, but not limited to, motors, pumps, and computer hardware need not be managed as a dangerous waste due to lead solder considerations. RCRA Compliance Support believes that equipment like this should be managed as non-dangerous wastes in accordance with standard industry practice.

On the other hand, this letter is intended to address situations when equipment is disassembled, or maintenance is performed which generates a waste stream. In these cases, the waste streams generated shall be evaluated to determine if they should be managed as a dangerous waste based on the lead solder weight percentages. The information in this letter can be refuted on a case-by-case basis through additional sampling. There is nothing preventing an individual facility from taking additional samples of a waste stream to obtain an alternate waste designation. 
The following waste streams were sampled: (1) pure lead solder, (2) small light bulbs (tail light lamps, annunciator panel lights), (3) fuses visibly containing lead solder, (4) computer circuit boards, (5) incandescent light bulbs (varied wattage), and (6) mercury vapor lamps. Attachment 1 presents the analytical data obtained from the sampling efforts. Attachment 2 presents the information that will be used to designate other waste matrices containing lead solder.

The sampling efforts that obtained the analytical results were completed in accordance with all applicable Resource Conservation and Recovery Act (RCRA) requirements. All equipment used in obtaining the samples were decontaminated prior to the sampling efforts in accordance with RCRA protocols. Proper chain-of custody and sample handling procedures were adhered to and all holding times were met. Preservatives were not used for these sampling efforts. In all cases except the mercury vapor lamps, the waste matrix met the Toxicity Characteristic Leaching Procedure (TCLP) particle size criteria in the field on the Hanford Facility by either cutting, smashing or shearing the waste. The mercury vapor lamp sampling effort was conducted at the Idaho National Engineering Laboratory so particle size reduction considerations are not known. Reducing the particle size in the field eliminated as much laboratory error as possible. The reduced particles were all well mixed and composited so that two identical samples would be submitted for analysis. Duplicate analyses help address the accuracy of the sampling effort.

All of the samples, including the mercury vapor lamps, are considered representative of Hanford Facility waste streams. The samples were submitted to the S-Cubed laboratory under contract through the Hanford Analytical Services Management group. The services of the Mobile Sampling Team were used to provide all of the equipment necessary and expertise to ship the samples off site to S-Cubed in addition to maintaining appropriate field log books of the sampling efforts. Once at S-Cubed, the samples were subjected to SW-846 method 1311, and followed with SW-846 Method 7421 for the lead analysis.

If the duplicate analyses for a waste matrix yielded sample results which appeared reproducible, they were averaged to obtain the value that is to be used for storage or disposal paperwork. If the results did not appear reproducible, the more conservative value will be used for designation purposes, unless additional sampling efforts are pursued to determine otherwise.

In summary, the data in Attachment 1 should be used for designating waste matrices identified in Attachment 1. As can be seen from the data in the table, pure lead solder would be designated as an extremely hazardous waste (EHW-0008). In addition, small light bulbs, lead solder fuses, computer circuit boards, incandescent light bulbs, and mercury vapor lamps would be designated as a dangerous waste (OW-D008). To obtain a lead designation status for waste matrices not identified in Attachment 1 , the wt\% of lead solder in the waste matrix should be compared to the table at the bottom of Attachment 2 . 
Page 3

August 3, 1993

Please reflect these changes in all storage and disposal paperwork from this point forward. The effective date of this letter will be used as the effective date for managing waste matrices containing lead solder as dangerous wastes. If you have questions or comments, please contact me on 376-3870.

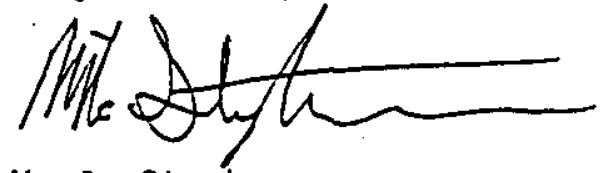

M. J. Stephenson

RCRA Compliance Support

$r \ln$

Attachments 
HNF-6273

Rev. 0

․ $88410-93-161$ ATTACHMENT 1

Page 1 of 1

Lead Solder Analysis Summary Table

\begin{tabular}{|c|c|c|c|}
\hline Waste Matrix & Sampte $1 \%(\mathrm{mg} / \mathrm{l})$ & 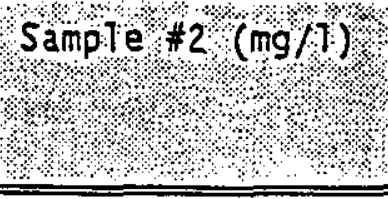 & $\begin{array}{l}\text { VaTue used for } \\
\text { storage or } \\
\text { disposal } \\
\text { paperwork (mg/l) }\end{array}$ \\
\hline Pure Lead Solder & 525 & 594 & 560 (avg.) \\
\hline $\begin{array}{l}\text { Small Light } \\
\text { Bulbs }\end{array}$ & 1.01 & 12.9 & 12.9 \\
\hline $\begin{array}{l}\text { Lead Solder } \\
\text { Fuses }\end{array}$ & 59 & 20.8 & 40 (avg.) \\
\hline $\begin{array}{l}\text { Computer Circuit } \\
\text { Boards }\end{array}$ & 372 & 31 & 372 \\
\hline $\begin{array}{l}\text { Incandescent } \\
\text { Light Bulbs }\end{array}$ & 0.538 & 22.7 & 22.7 \\
\hline $\begin{array}{l}\text { Mercury Vapor } \\
\text { Lamps }\end{array}$ & $44.7^{*}$ & $40.2^{\circ}$ & 42.5 (avg.) \\
\hline
\end{tabular}

* Results received from the Idaho National engineering Laboratory (INEL).

Toxicity Characteristic regulatory thresholds for lead (0008):

$$
O W=5.0 \mathrm{mg} / \mathrm{EHW}=500 \mathrm{mg} / 1
$$


HNF-6273

Rev. 0

88410-93-161
ATTACHMENT 2
Page 1 of 1

Calculation Summary

The following summary is provided for matrices that require evaluation for lead solder designation, but were not addressed during the sampling efforts. This methodology will be used to complete waste designations in those matrices.

An averaged TCLP analysis for the pure lead solder sample yielded a result of $560 \mathrm{mg} / 7$ of lead. The pure lead solder consisted of three typical tin/lead ratios: tin/lead at $63 / 37,60 / 40$, and $50 / 50$ wt\%. A ratio was used to calculate the amount of lead solder in a waste matrix that would cause it to exceed the Toxicity Characteristic regulatory thresholds of $5 \mathrm{mg} / 1$ (dangerous waste) and $500 \mathrm{mg} / 1$ (extremely hazardous waste) for lead. For the purposes of these calculations, it is assumed that other matrices containing lead solder will leach lead according to the following ratio:

$100 \mathrm{wt} \%$ lead solder $560 \mathrm{mg} / \mathrm{l}$ of lead
$X$ or $Y$

$\mathrm{mg} / \mathrm{l}$ designation limit for lead

$X=(100 \mathrm{wt} \%$ solder $/ 560 \mathrm{mg} / 1 \mathrm{~Pb}) \times 5 \mathrm{mg} / 1$ OW designation limit for $\mathrm{Pb}$

$X=0.893 w t \%$ solder for DW-D008 designation limit

$Y=(100 \mathrm{wt} \%$ solder $/ 560 \mathrm{mg} / \mathrm{Pb}) \times 500 \mathrm{mg} / \mathrm{l}$ EHW designation limit for $\mathrm{Pb}$

$Y=89.3$ wt\% solder for EHW-D008 designation limit

\begin{tabular}{|c|c|}
\hline 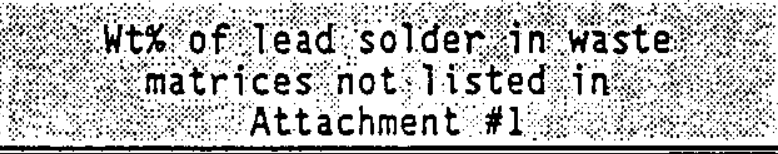 & oestgnations status for tead \\
\hline Less than 0.893 & Non-regulated \\
\hline $\begin{array}{c}\text { Greater than or equal to } 0.893 \text {, but } \\
\text { less than } 89.3\end{array}$ & DW-0008 \\
\hline Greater than or equal to 89.3 & $E H W-0008$ \\
\hline
\end{tabular}


HNF-6273

Rev. 0

E-6 


\section{DISTRIBUTION SHEET}

To

R. B. Wilkinson

Project TitleMork Order

Data Quality objectives Process for Designation of $\mathrm{K}$ Basins

\section{From}

J. L. Westcott
Page 1 of 1

Date May 11, 2000

EDT No 621183

ECN No.

\section{Name}

R. B. Wilkinson

J. I. Westcott

R. R. Connolyy

J. R. James

M. A. Casbon

W. S. Thompson

R. W. Jackson

J. M. Atwood

B. D. Shllperoort

C. D. Lucas

D. J. Watson

P. T. DaY

J. H. Wicks

J. H. Zimmerman

N. O. Hinojosa

G. C. Triner

\begin{tabular}{|c|c|c|c|c|}
\hline MSIN & $\begin{array}{l}\text { Text } \\
\text { With All } \\
\text { Attach }\end{array}$ & Text Only & $\begin{array}{l}\text { Attach } / \\
\text { Appendix } \\
\text { Only }\end{array}$ & $\begin{array}{l}\text { EDT/ECN } \\
\text { Only }\end{array}$ \\
\hline $\mathrm{R} 3-11$ & & & & $x$ \\
\hline$T 4-05$ & $x$ & & & : \\
\hline$T 3-04$ & & & & $x$ \\
\hline $\mathrm{L} 6-06$ & & & & $x$ \\
\hline$T 2-05$ & $x$ & & & \\
\hline $\mathrm{H} 9-03$ & $x$ & & & \\
\hline$x 5-54$ & $x$ & & & \\
\hline $\mathrm{HO}-02$ & & & & $x$ \\
\hline $\mathrm{T} 2-05$ & $x$ & & & $\because$ \\
\hline$\times 3-74$ & & & & $x$ \\
\hline$\times 3-79$ & $x$ & & & \\
\hline R3-11 & $x$ & & & $\ldots$ \\
\hline$x 3-71$ & . & & & $x$ \\
\hline$\times 3-67$ & $x$ & & & 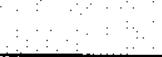 \\
\hline$\times 3-67$ & $x$ & & & $\because$ \\
\hline$T 3-04$ & & & & $x$ \\
\hline
\end{tabular}

